\title{
APPLICATION OF VARIATIONAL SYNTHESIS TO SOLUTIONS OF MULTIDIMENSIONAL NEUTRON TRANSPORT PROBLEMS
}

\author{
A THESIS \\ Presented to \\ The Faculty of the Graduate Division \\ by \\ William Ronald Cobb \\ In Partial Fu.lfillment \\ of the Requirements for the Degree \\ Doctor of Philosophy \\ in the School of Nuclear Fngineering
}

\author{
Georgia Institute of Technology \\ Deceraber, 1970
}


APPLICATION OF VARIATIONAL SYNTHESIS TO SOLUTIONS OF

MULTIDIMENSIONAL NEUTRON TRANSPORT PROBIEMS

Approved:

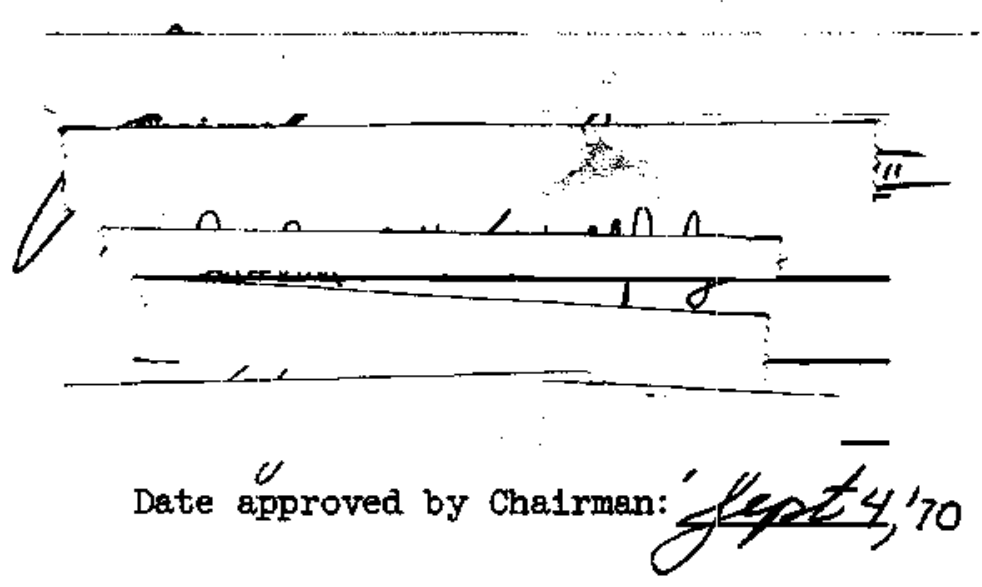


In presenting the dissertation as a partial fulfiliment of the requirements for an advanced degree from the Georgia Institute of Technology, I agree that the Library of the Institute shall make it available for inspection and circulation in accordance with its regulations governinis materials of this type. I agree that permission to copy from, or to publish from, this dissertation may be granted by the professor under whose direction it was written, or, in his absence, by the Dean of the Graduate Division when such copying or publication is solely for scholarly purposes and does not involve potential financial gain. It is understood that any copying from; or publication of, this dissertation which involves potential financial gain will not be allowed without written permission.

$7 / 25 / 68$ 


\section{ACKNOWLEDGEMENTS}

It is a pleasure to acknowledge the assistance of at least some of the many people who have had an interest in the work described in this thesis. The advice and encouragement of Dr. J. D. Clement, who served as thesis advisor, is gratefully acknowledged. The members of the reading committee, Drs. W. W. Grahom, J. M. Kallfelz, and M. P. Stallybrass, have contributed to this work both through their review of the manuscript and through several discussions. Dr. C. J. Roberts, Director of the School of Nuclear Engineering, has been very helpful, particularly in regard to the arrangements for this investigation to be done in absentia. Special permission was received from the Graduate Division to use centered page numbers so that this report could be published by the Oak Ridge National Laboratory.

This research was performed at the Oak Ridge National Laboratory, operated by Union Carbide Corporation for the United States Atomic Energy Commission. Many members of the staff of ORNL have provided help and encouragement during the pursuit of this investigation. I am particularly grateful to Dr. C. W. Craven, Jr, for providing the original stimulus to work in this area, for serving as a member of the reading committee, and for showing continued interest in this work and its outcome. Dr. A. M. Perry, Head of the Reactor Analysis Departinent, made this study possible by making available necessary time and facilities. Discussions with and criticisms from Drs. D. R. Vondy, M. I. Tobias and J. D. Jenkins have been both helpful and stimulating. 
The patience and hard work of Mrs. Margaret Wilson is largely responsible for both the draft of this thesis and the final polished manuscript, and is greatly appreciated.

I express my sincere appreciation to my wife, Jeanette, for her understanding and her inspiration throughout my graduate career, and to my daughter, Anne, whose timely arrival contributed in large measure to the early completion of this work. No acknowledgement could be complete without an expression of gratitude to my parents, Mr. and Mrs. R. O. Cobb, Sr., without whose guidance in earlier years this work would never have begun. 
TABLE OF CONTENTS

Page

ACKNOWLEDGMENTS . . . . . . . . . . . . . . . . .

IIST OF TABLES

vi

LIST OF ILLUSTRATIONS . . . . . . . . . . . . . . . . vi vi

SUMMARY . . . . . . . . . . . . . . . . . . . . . ix

Chapter

I. INTRODUCTION . . . . . . . . . . . . . . . .

Background

Purpose of the Research

II. THEORY . . . . . . . . . . . . . . . . .

The Variational Synthesis Method

III. A VARIATIONAL SYNTHESIS APPROXIMATION FOR THE NEUTRON TRANSPORT PROBLEM . . . . . . . . . . . . . . .

The Boltzmann Transport Equation

The Energy Approximation

The Spatial Symthesis Approximation

Method of Solution

Summary of the Approximation Procedure

IV. COMPUTER CODES USED $, \cdot . \cdot, \cdot, \cdot, \cdot, \cdot, \cdot, \cdot \cdot$

The TS2 Experimental Computer Code

Computer Codes Used for Obtaining Direct Solutions

Guidelines Used in Comparisons

V. DISCUSSION OF RESULTS . . . . . . . . . . . .

Types of Problems Considered

Test Problem Results

VI. CONCLUSIONS . . . . . . . . . . . . . . 
TABLE OF CONTENTS (Continued)

Page

APPENDICES ............................... 124

A. A FUNCTIONAL FOR THE VARIATIONAL CHARACTERIZATION

OF THE NEUTRON TRANSPORT PROBLEM . . . . . . . . . * 125

B. REDUCED EQUATIONS FOR THE THREE-DIMENSIONAL CASE . . . 140

C. REFLECTED BOUNDARY CONDITIONS . . . . . . . . . 145

BIBLIOGRAPHY . . . . . . . . . . . . . . . . . 151

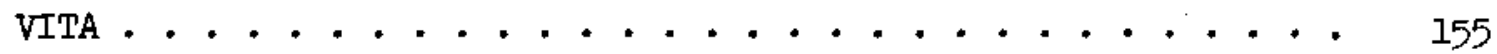




\section{LIST OF TABLES}

Table

Page

1. Results for Fixed Source Problem One with $S_{4}$ and $S_{6}$ Angular Quadrature ................. 74

2. Results for Fixed Source Problem One with Analytic Base Functions . . . . . . . . . . . . . 78

3. Results for Eigenvalue Problem one . . . . . . . . 81

4. Results for Fixed Source Problem Two . . . . . . . . . . 84

5. Results for Eigenvalue Problem Two . . . . . . . . . 88

6. Channel Wiaths for Calculations of Problem Three . . . . 94

7. Results for Problem Three . . . . . . . . . 95

8. Results for Problem Four . . . . . . . . . . . . . 104 


\section{LIST OF ILLUSTRATIONS}

Figure

Page

1. Geometry for Typical Two-Dimensional Rectangular Reactor . . . . . . . . . . . . . . . . . .

2. Geometry and Macroscopic Constants for Problem One . . .

3. Scalar Flux Along Diagonal for Fixed Source Problem One . . . . . . . . . . . . . . . . . . . .

4. Percentage Deviations in Scalar Flux Along Diagonal . . 79

5. Geometry and Macroscopic Constants for Problem Two . . . 83

6. Scalar Flux Along Diagonal for Fixed source Problem Two . . . . . . . . . . . . . . . . . . . . .

7. Scelar Flux Along Diagonal for Eigenvalue Problem Two . . . . . . . . . . . . . . . . . . .

8. Axial Scalar Flux Through the Center of the Absorber Region . . . . . . . . . . . . . . . . . .

9. Geometry and Macroscopic Constants for Problem Three . . . . . . . . . . . . . . . . . .

10. Horizontal Scalar Flux Through the Upper Axial Zone . . . 97

11. Horizontal Scalar Flux at the Axial Zone Interface, Series One and Three ..................

12. Horizontal Scalar Flux at the Axial Zone Interface, Series Two . . . . . . . . . . . . . . 100

13. Geometry and Macroscopic Constants for Problem Four . . . 102

14. Axial Scalar Flux Through the Seed Regilon . . . . . . 106

15. Projective Plot of $S_{2}$ Scalar Flux . . . . . . . . 107

16. Scalar Fluxes Along Bottom Edge, Problem Four . . . . . . 109

17. Scalar Fluxes Along Left Edge, Problem Four . . . . . . 110

18. Geometry and Macroscopic Constants for Problem Five . . . 112 


\section{LIST OF ILLUSTRATIONS (continued)}

Figure

Page

19. Scalar Flux Along Left Edge, Problem Five . . . . . . 114

20. Scalar Flux Along Bottom Fdge, Problem Five . . . . . 115

21. Convex, Two-Region Reactor Geometry . . . . . . 130 


\section{SUMMARY}

The variational synthesis method has been applied to the steadystate Boltzmann neutron transport equation in two- and three-dimersional rectangular geometry. In this application the energy dependence has been treated in the standard multigroup energy approximation, the dependence along all but a single remaining spatial variable has been treated by a multichannel discontinuous spatial synthesis approximation and the angular dependence has been treated by a discrete ordinates approximation. All approximate treatments of these variables have been developed consistently from a variational principle by the standard trial function reduction procedure, applied sequentially to the energy variable, the spatial variables and the angular variable. The approximate method as developed in this application allows the user to obtain approximate solutions to the transport equation by combination of the solutions to related problems of lower dimensionality.

An experimental computer code has been written by the author for use in obtaining approximate solution to the two-dimensional, one energy group problem with isotropic scattering. The experimental computer code has been used to obtain approximate solutions to several test problems of the type encountered in the reactor field and the accuracy and comparative cost of these approximate solutions has been determined by comparison to consistently obtained direct solutions to the same problems. Both fixed source and eigenvalue problems, as well as problems with both vacuum and reflected boundary conditions, have 
been investigated. Results of the test problems have indicated that the method, as developed, is capable of producing accurate approximate transport solutions at a fraction of the cost of the direct solutions, and with accuracy superior to the diffusion approximation. 
CHAPTER I

INTRODUCTION

\section{Background}

The description of the steady-state neutron behavior in nuclear reactors requires the specification of dependence of the neutron density on six independent variables -- three spatial, two direction and one energy. Although this specification cannot be described analytically in realistic reactor situations, it is conceptually possible to attack the problem by straightforward finite difference or orthogonal polynomial expansion methods. In the sense that these direct methods approach the exact description in the limit as the finite differences approach zero and as the number of terms in the expansion becomes infinite, they are usually considered to be "exact".

Practical limitations on the speed and storage capacity of the current generation of computers prevent the implementation of these direct methods, however, and the reactor physicist must resort to methods yielding approximate descriptions. These approximate methods usually involve simplifying assumptions, about the physical model or about the equations themselves, which effectively remove one or more independent variables from consideration.

For example, if the physical process describing the neutron transport is assumed to be equivalent to a diffusion process, then the two angular direction variables are removed from consideration, and the neutron behavior can be described by only four independent variables. 
A straightforward finite difference approach may then be implemented to obtain a description of the neutron behavior based on this simplified model. The "diffusion approximation" has been, and continues to be, the most widely used method in describing the neutron behavior in nuclear reactors. While not yielding any information about the anjular dependence of neutron distribution, it does provide an approximation to the scalar neutron flux, which is of primary importance in reactor core design work. The diffusion theory solution is valid at points within the reactor where the angular neutron flux is approximately isotropic. Near external boundaries and internal material interfaces, however, anisotropy of the angular flux can cause the diffusion approximation to be highly inaccurate.

Alternatively, the angular variables may be retained and the neutron behavior assumed independent of one or two of the spatial variables. These variables are then omitted from the governing equations and a two- or one-dimensional transport solution may be obtained by the direct methods mentioned above. If the extent of the spatial variables omitted from the governing equations is very large, then these lower dimensional solutions can be expected to give an adequate approximate description of the neutron behavior at points far removed from boundaries of the omitted variables.

A more accurate method of obtaining approximate solutions by the removal of one or more variables is the synthesis method, which has evolved in the reactor field over the past decade primarily for diffusion theory applications. The term synthesis, in its broadest application, refers to a wide class of "trial function" approximation 
techniques utilizing some form of the general method of weighted residuals. More specifically, the term variational synthesis refers to the use of an approximation technique of the variational calculus known as the Indirect, or Kantorovich, ${ }^{1}$ method. The basic idea behind the synthesis method is to incorporate some a prlori knowledge about the dependence of the neutron density on the variables to be removed. This a priori knowledge may be due to the experience or intuition of the reactor physicist, or may be obtained from auxiliary calculations which are readily performed.

Suppose, for example, that the reactor physicist has at hand a set of functions, $f_{k}(x), k=1,2, \ldots, k$, which are known to be capable of describing the dependence of some unknown function, $\Psi(x, y)$, on the independent variable $x$. To utilize the synthesis method to obtain an approximation to the unknown function, $\Psi(x, y)$, an approximate function or "trial function", $\psi(x, y)$, is formed as

$$
\psi(x, y)=\sum_{k=1}^{K} \phi_{k}(y) f_{k}(x)
$$

when the $f_{k}(x)$ are the known "base functions" and the $\phi_{k}(y)$ are unknown "mixing functions" which must be determined. The synthesis method then prescribes a way of determining the unknown mixing functions $\phi_{\mathbf{k}}(\mathrm{y})$. Having determined the functions $\phi_{\mathbf{k}}(\mathrm{y})$, the approximate solution is obtained by recombination via Equation 1.1.

There is an interesting parallel between the development of the synthesis method in the reactor field on the one hand and the increased speed and capacity of each new generation of computers on the other. 
The capacity of the earliest computers was so limited that the calculation of the neutron distribution was restricted to the one-dimensional diffusion-theory approximations. Attempts to utilize the available one-dimensional solutions to obtain approximate two-dimensional results were widespread. ${ }^{2}$ The most commonly used method was termed "Buckling Iteration", and although not called synthesis at that time, has been shown to correspond to a particular application of the synthesis method. 3

With the increased capability of the next generation of computers, direct solutions to the two-dimensional diffusion approximation became practical. Utilizing the available two-dimensional diffusion-theory solutions, many attempts were made to obtain approximate three-dimensional diffusion-theory solutions, $4,5,6,7$ the direct solutions being impractical. Indicative of the success achieved in these attempts is the fact that Meyer's symthesis method, ${ }^{6}$ and Wachspress' generalization of Meyer's method, 7 were widely used for design calculations at Bettis and Knolls Laboratory, 8,9

When the computer capacity had advanced to the point where direct solutions to the three-dimensional diffusion approximation were practical, the approximate symthesis methods were still used in design situations where many calculations were required. This usage is still found, even for obtaining approximate two-dimensional diffusion solutions, $10,11,12$ because the cost of obtaining the approximate synthesis solution is much less than that of obtaining the direct solutions. The usefulness of the synthesis method is thus seen to be twofold: at a time when higher dimensional solutions were impractical, it provided 
a means of obtaining approximate solutions by utilizing the available lower dimensional solutions as base functions, and when the higher dimensional solutions became available it provided a fast and accurate approximate solution for use in design situations where many calculations were required and where a direct solution for each one would be unnecessarily expensive.

Examples of the successful application of the synthesis method to other problems in diffusion theory are plentiful. The removal of all or some of the spatial variables from the time dependent diffusion equation has been shown repeatedly to give good approximate results with large savings in computational time. Successful applications have been made to both fast transient $13,14,15,16$ and depletion-type problems. $8,11,17$ The removal of the energy variable by a synthesis approximation has also been shown to give good results in comparison to both experiments and to direct multigroup calculations. $18,19,20$ It is interesting in this respect to point out that the multigroup approximation, which is the standard direct treatment for the energy variable, can be considered as a particular case of a synthesis approximation. 21,22

The current problem of obtaining multidimensional transport solutions is much like the problem faced a decade ago, with regard to obtaining multidimensional diffusion-theory solutions. Fast and ej:icient solutions to the one-dimensional transport equation are obta:ined routinely but direct two-dimensional solutions are expensive and time consuming, and their frequent use in routine design situations is impractical. Computer codes for obtaining direct solutions to the 
three-dimensional transport equation are now in the very early stages of development. The Monte Carlo method is capable, at present, of ylelding solutions to the three-dimensional transport equation but the statistical nature of this method often requires excessive amounts of computer time.

The success achieved in applying the synthesis method to the diffusion-theory problem quite naturally suggested its application to the more difficult problem of transport theory. Davis and Kaplan 23 first reported an attempt to obtain a transport solution by using the synthesis method to remove a spatial variable from the two-dimensional problem. In this work the synthesis method was applied to the discrete ordinates approximation to the transport equation and the results were described by the authors as both encouraging and discouraging. The results were encouraging because they were more accurate than the diffusion-theory solution, and less expensive to obtain than the direct solution to the discrete ordinates equations. They were discouraging, however, because the selection of base functions was not as clearly defined as for diffusion-theory applications, and because the results failed to reproduce certain characteristics of the direct solution to the problem. These characteristics, called "ray effects", will be discussed in more detail in Chapter $V$.

Kaplan, Davis, and Natleson ${ }^{24}$ applied the synthesis method to the trensport equation to remove the angular variables in a technique termed "Space-Angle Synthesis" (SAS). After initially successful applications of the method to Milne's problem, Natleson proposed a "Strategy for the Application of Space-Angle Synthesis to Practical 
Problems of Neutron Transport".25 In obtaining solutions to practical two-dimensional problem, Natleson's strategy was to obtain the angular base functions from a high-order, two-dimensional transport calculation for a problem of interest. He then proposed using these base functions to synthesize fluxes for "similar" two-dimensional problems of interest. Noting that the SAS calculation was less sensitive to the spatial mesh structure than true transport calculations, he proposed that the method be incorporated into existing diffusion theory codes to allow the transport synthesis to be performed only within energy groups where diffusion theory gave a poor approximation.

While there is, no doubt, a class of problems for which the SAS method will provide relatively accurate and inexpensive calculaticns, one useful (and perhaps the most useful) capability of the synthesis method appears to have been neglected: That of extending the computational capability to allow approximate solutions to problems for which direct solutions are unattainable. The capability of three.. dimensional transport theory solutions is a case in point. Following Natleson's strategy, base functions for a three-dimensional problem must be obtained from a three-dimensional transport code. Even axsuming that such base functions could be easily obtained, the system of equations for the mixing functions would be three-dimensionel; and as such, very difficult to solve.

The angular base functions from two-dimensional calculations cannot be used in the SAS method to symthesize three-dimensional transport solutions. Angular fluxes from, say, an $x-y$ transport calcuitation are symmetric about the $\mathrm{x}-\mathrm{y}$ plane and their use in a three-dimensional 
trial function with angular dependent base functions, will, of course, produce synthesized fluxes which are also symmetric.

$0^{\prime}$ Reilly ${ }^{26}$ has reported the development of a symthesis method in which angular base functions from two-dimensional calculations could be used to synthesize three-dimensional transport solutions. To avoid the possibility of obtaining symmetric approximate solutions, he modified the SAS method so that the mixing functions, as well as the base functions, were dependent on the angular variable. This angular dependence of the mixing functions was accomplished by segmentation of the unit sphere into octants, in a discrete-ordinates-like manner. Since, however, only eight segments are proposed, it is questionable whether the results, at least along the third dimension, would be any more accurate than diffusion theory. No numerical results have been offered in support of $O^{\prime}$ Reilly's method.

An interesting and somewhat similar method has been proposed recently by Natleson and Gelbard. 27 By choosing base functions dependent on angle and on two spatial variables, their method allows one to use transport base functions in a diffusion-theory synthesis. The method is similar to $O^{\prime} R^{\prime} i l y^{\prime} s$ in that the results along the third spatial variable are expected to have accuracy of the order of the diffusion approximation.

Other authors have reported application of the synthesis method to the transport equation. Zwibel and Bowes ${ }^{28}$ applied the method to remove the spatial variable from the one-dimensional transport equation, and Luco $^{29}$ developed a method of removing the spatial and angular variables from the time-dependent transport equation. More recently, 
Lancefield ${ }^{30}$ applied the method to remove the energy variable from the one-dimensional equation.

\section{Purpose of the Research}

It was the purpose of the research described in this thesis to:

(1) Develop a variational synthesis technique, applicable to both two- and three-dimensional problems, which would allow approximate transport solutions to be obtained with greater accuracy than the aiffusion approximation.

(2) To incorporate into the approximate method various refinements which have recently been developed and used successfully in the application of the synthesis method to diffusion-theory problems, and to determine their applicability to the transport problem.

(3) Develop an experimental computer program capable of obtaining the approximate solution for the two-dimensional transport problem.

(4) Demonstrate the applicability of the method by comparison of the accuracy and cost of the approximate two-dimensional solutions to direct transport and diffusion-theory solutions.

Demonstration of the applicability of this approximate method to several sample problems allows an assessment of the practicality of developing a computer program utilizing the method for use as an actual design tool. 
CHAPTER II

THEORY

The Veriational synthesis Method

The use of the variational calculus as a tool for obtaining approximations in reactor theory is generally attributed to selengut, ${ }^{3}$ although specific applications to the criticality problem by Pryce and by Fuchs were reported as early as 1943.31 It was the work of Selengut, however, which systematized the application of the variational calculus to a wide variety of problems in reactor theory. Kaplan ${ }^{32}$ first utilized the indirect, or Kantorovich, method of the variational calculus for approximating multidimensional diffusion-theory solutions using as base functions solutions of lower dimensionality. This and various subsequent works $13,23,24$ of Kaplan are largely responsible for the popularity of the term variational synthesis, and, indeed, the popularity of the method itself.

Outline of the Variational Synthesis Method

Prior to the development of the variational synthesis method for the neutron transport problem, it is instructive to outline the use of the method in a brief, but general, example.

Suppose the state of some physical system is governed by the equation

$$
\mathrm{T}(\mathrm{x}, \mathrm{y}, \hat{\Omega}) \Psi(\mathrm{x}, \mathrm{y}, \hat{\Omega})=\mathrm{S}(\mathrm{x}, \mathrm{y}, \hat{\Omega})
$$


supplemented by appropriate boundary conditions. Here $\Psi$ is the unknown state function, $\mathrm{T}$ is the linear system operator, and $\mathrm{S}$ is the source or forcing function. If the system operator is not self-adjoint, then there is an auxiliary or adjoint state description

$$
\mathrm{T}^{*}(\mathrm{x}, \mathrm{y}, \hat{\Omega}) \Psi^{*}(\mathrm{x}, \mathrm{y}, \hat{\Omega})=\mathrm{S}^{*}(\mathrm{x}, \mathrm{y}, \hat{\Omega})
$$

where $\psi^{*}$ is the unknown adjoint state function, and $S^{*}$ is the adjoint source. $T^{*}$ is the operator adjoint to the system operator $T$ by the usual definition

$$
\int \mathrm{dx} \int \mathrm{dy} \iint \mathrm{d} \hat{\Omega} \mathrm{uT}^{*} \mathrm{v}=\int \mathrm{dx} \int \mathrm{dy} \iint \mathrm{d} \hat{\Omega} \mathbf{v T u}
$$

for arbltrary functions $u$ and $v$. Subsequent discussion will refer to Equations 2.1 and 2.2 as the forward and adjoint system equations respectively, and to their solutions as the forward and adjoint state functions.

To utilize the variational synthesis method in obtaining an. approximation to the unknown state function, $\Psi$, a functional $\mathscr{F}\left[\phi, \phi^{*}\right]$ must be obtained such that the variational principle

$$
8 \%=0
$$

is a complete alternate description of the physical problem within the class of functions, $\phi$ and $\phi^{*}$, forming its domain. If the boundary conditions of Equation 2.1 are essential boundary conditions, in the sense of the calculus of variations, then the domain of includes only those functions satisfying these conditions. If the boundary conditions of Equation 2.1 are natural boundary conditions, then the 
domain may include functions not satisfying those conditions, although it is often advantageous to consider only those which do satisfy them. The functional chosen here is of the Roussopoulos type, ${ }^{33}$ which can be derived by the method of Pomraning ${ }^{34}$ and Lewins ${ }^{35}$ or otherwise obtained as proposed by Becker. ${ }^{36}$ The Roussopoulos functional has, as its Euler equations, the forward and adjoint system equations (Equations 2.1 and 2.2), and is expressed as

$$
\Re\left[\phi, \phi^{*}\right]=\int \mathrm{dx} \int \mathrm{dy} \iint \mathrm{d} \hat{\Omega}\left\{\phi^{*}[S-T \phi]+S^{*} \phi\right\}
$$

where, for the sake of clarity, the arguments of the functions in the integrand are omitted, and assumed understood. The details of the demonstration that the functional is a valid variational characterization of the system equations are given in Appendix A.

Having an appropriate functional, an approximation to the state functions $\Psi$ is obtained by forming the trial function

$$
\phi(\mathrm{x}, \mathrm{y}, \hat{\Omega})=\sum_{\mathbf{k}=1}^{2} \phi_{\mathbf{k}}(\mathrm{y}, \hat{\Omega}) \mathrm{f}_{\mathbf{k}}(\mathrm{x})
$$

A similar trial function for the adjoint must also be formed as

$$
\phi^{*}(\mathrm{x}, \mathrm{y}, \hat{\Omega})=\sum_{\mathrm{k}=1}^{2} \phi_{\mathrm{k}}^{*}(\mathrm{y}, \hat{\Omega}) \mathrm{f}_{\mathrm{k}}^{*}(\mathrm{x})
$$

Here $f_{k}(x)$ and $f_{j}^{*}(x)$ are known forward and adjoint base functions which are considered capable of describing the $x$ variations of the state functions $\Psi$ and $\Psi^{*}$ respectively. The $\phi_{k}(y, \hat{\Omega})$ and $\phi_{j}^{*}(y, \hat{\Omega})$ are the unknown forward and adjoint mixing functions. To obtain the approximate 
solutions, the trial functions $\phi$ and $\phi^{*}$ are inserted into the functional and the integration over the base function variable, $x$, performed.

Resulting from the procedure is a reduced functional $F$ depending on the mixing functions $\phi_{k}$ and $\phi_{j}^{*}$ :

$$
\mathscr{F}\left[\phi_{k}, \phi_{j}^{*}\right]=\sum_{j=1}^{2} \int \mathrm{dy} \iint \mathrm{d} \hat{\Omega}\left\{\phi_{j}^{*}\left[S_{j}-\sum_{k=1}^{2} T_{j k} \phi_{k}\right]+s_{j}^{*} \phi_{j}\right\}
$$

where the following definitions have been employed:

$$
\begin{gathered}
\left.S_{j} y, \hat{\Omega}\right)=\int d x f_{j}^{*}(x) s(x, y, \hat{\Omega}) \\
T_{j k}(y, \hat{\Omega})=\int d x f_{j}^{*}(x) T(x, y, \hat{\Omega}) f_{k}(x) \\
S_{k}^{*}(y, \hat{\Omega})=\int d x S^{*}(x, y, \hat{\Omega}) f_{k}(x)
\end{gathered}
$$

To obtain equations governing the mixing functions $\phi_{k}(y, \hat{\Omega})$, the variational principle is imposed on the reduced functional by requiring the variational derivative with respect to the adjoint mixing functions, $\phi_{j}^{*}(y, \hat{\Omega})$, to vanish. If the reduced functional is to be stationary, then the variational derivative with respect to the $\phi_{k}(y, \hat{\Omega})$ must also vanish, and a set of equations determining the $\phi_{j}^{*}(y, \hat{\Omega})$ will result. Thus, imposing the condition

$$
\delta \mathscr{F}_{\phi_{j}^{*}}=0, \quad j=1,2
$$

yields the Euler equations determining the $\phi_{\mathbf{k}}$ as 


$$
\mathrm{s}_{j}-\sum_{\mathbf{k}=1}^{2} \mathrm{~T}_{\mathbf{j k}} \phi_{\mathbf{k}}=0, \quad j=1,2
$$

and requiring that

$$
{ }_{\phi_{k}}=0, \quad \mathrm{k}=1,2
$$

yields the Euler equations determining the $\phi_{j}^{*}$ as

$$
S_{k}^{*}-\sum_{j=I}^{2} T_{k_{j}}^{*} \phi_{j}^{*}=0, \quad k=I, 2
$$

These equations may be written compactly using matrix notation

as

$$
\mathrm{T}(\mathrm{y}, \hat{\Omega}) \phi(\mathrm{y}, \hat{\Omega})=\mathrm{s}(\mathrm{y}, \hat{\Omega})
$$

and

$$
\mathrm{T}^{*}(\mathrm{y}, \hat{\Omega}) \phi^{*}(\mathrm{y}, \hat{\Omega})=\mathrm{S}^{*}(\mathrm{y}, \hat{\Omega})
$$

where the elements of the matrix operator $\mathrm{T}$ are the reduced operators $\mathrm{T}_{j \mathrm{k}}$ and those of the source vectors are $s_{j}$ and $\mathrm{S}_{\mathrm{k}}^{*}$ as given in Equations 2.9 and 2.11. The unknowns are thus column vectors whose elements are the mixing functions $\phi_{\mathbf{k}}$ and $\phi_{j}^{*}$. The approximate solution $\phi(x, y, \hat{\Omega})$ is then obtained by solving the matrix Equation 2.16 for the mixing coefficients and combining these with the base functions by use of the trial function expression of Equation 2.6. If an approximation for the adjoint state function is also desired, it is obtained in a similar manner by solving Equation 2.17. 
In this example, the variational synthesis method has provided a means of removing the independent variable $\mathrm{x}$ from the system equations by the incorporation of some a priori knowledge of the dependence of the true solution on $x$. This a priori knowledge is, of course, contained in the base functions $f_{k}(x)$ and $f_{j}^{*}(x)$, and the reduced Equation 2.16, is a set of two coupled equations containinis only the independent variables $\mathrm{y}$ and $\hat{\Omega}$. In the more general case where the trial functions of Equations 2.6 and 2.7 contain $K$ base functions, the resultant approximate equations for the forward and adjoint mixing functions will each form a set of $k$ coupled equations. Note that the solution for the adjoint mixing functions is not required in approximating the state function $\Psi$, but that adjoint base functions $f_{j}^{*}(x)$ must be utilized.

Selection of Base Functions

The accuracy of the approximate solution $\phi$, obtained by the variational synthesis method, is strongly dependent on the choice of base functions $f_{k}(x)$. If the choice of base functions is such that the true solution can be expressed exactly as some combination of the base functions, as in Equation 2.6, then the resultant synthesized solution will be exact. If, however, the base functions bear little resemblance to the dependence of the true solution on the independent variables they are chosen to represent, then the synthesized solution can be highly inaccurate. If an approximation to the forward solution is of primary interest, the choice of adjoint base functions is less critical since they enter into the approximation of $\Psi$ as weight functions. Similarly, if an approximate adjoint solution is of primary 
interest, the choice of adjoint base functions must be well made, and choice of forward base functions is less critical.

Experience in applying the synthesis method to the diffusiontheory problem has shown that if, within the set of base functions being utilized, there is contained one or more which are representative of the true $\mathrm{x}$ variation for each portion of the independent variables which are not removed, then an adequate approximate solution can be obtained. The difficulty in obtaining the synthesis solution, however, increases roughly as the square of the number of base functions included. If the number of base functions necessary to obtain an adequate approximation is very large, then the cost of obtaining the synthesis solution will approach the cost of the direct solution.

This situation, as well as the situation encountered in some problems when the choice of which base functions to use is not clearly defined, has prompted a generalization of the variational synthesis method to allow the use of discontinuous trial functions. Discontinuous Trial Functions

If the synthesis method is to provide a fast, as well as accurate approximation, it is important not only to include base functions which characterize the true solution along the variables to be removed, but also to exclude any which fail to do so. To this end, it is edvantageous to modify the method so as to allow certain of the base functions to be used only over the portions of the remaining variables where they are most representative of the true solution. The dif. ficulty of obtaining the approximation can tihereby be reduced, at the expense of introducing discontinuities in the approximate solution at points where the base functions are switched. 
This modification may be accomplished by including the boundary and continuity conditions of the system in the functional by use of Lagrange multipliers as advocated by Buslik ${ }^{37}$ and by Pomraning. 34 By using the method of Lagrange multipliers, the modified functional has as its Euler equations, the system equations and their associated boundary and continuity conditions. Details of the modification of the Roussopoulos functional to allow the extension of the domain are given in Appendix A.

If the nature of the problem to be solved by the synthesis method is such that a clear choice of base functions is indicated, then the straightforward application of the method generally yields accurate results. In obtaining approximate multidimensional solutions using base functions generated in auxiliary lower dimensional calculations, the choice of which lower dimensional calculation to perform is often not clearly defined. It is also true that a large fraction of the total cost in obtaining the synthesis solution may be due to the expense of obtaining base functions. In such situations it is advantageous to utilize the variational synthesis method in such a way that each biase function is utilized in the best possible manner. The concept of a "multichannel" synthesis, first introduced by Wachspress ${ }^{7}$ and later refined by Wachspress and Becker, ${ }^{38}$ has beer. proposed for use in applications of the synthesis method to diffusion theory to avold this type of problem. Using the multichannel idea, the variable removed by the base functions is segmented into "channels" and the synthesis method is allowed to $\mathrm{mix}$ the base functions differently within each segment so as to obtain a more accurate approximate solution. Using this idea in 
the previous example would require the trial function of Equation 2.6 to be written, for the case of $N$ channels, as

$$
\phi(x, y, \hat{\Omega})=\sum_{n=1}^{N} \Delta_{n} \sum_{k=1}^{2} \phi_{n k}(y, \hat{\Omega}) f_{k}(x)
$$

with a similar modification for the adjoint trial function. In this expression the function $\Delta_{n}$ is a step function which is unity over the nth channel and zero elsewhere. The mixing functions now have a plecewise dependence on the variable removed, as indicated by the additional subscript n. Following the same procedure as before, the resultant matrix equations determining the mixing coefficients, $\phi_{n k}(y, \hat{\Omega})$, will be identical to Equation 2.16, except that the operator $T$ is now an NK square matrix and the column vectors $\phi(y, \hat{\Omega})$ contain NK elements. The synthesis method without reference to the multichannel idea is, of course, the special case for which $\mathrm{N}=1$.

With the introduction of the multichannel concept, the number of unknown mixing functions to be determined and the difficulty of obtaining them by solution of Equation 2.16 has been increased. The sensitivity of the resulting approximate solution on the choice of base functions and on the number of base functions has been reduced. The trade-off between the cost of obtaining the base functions and the cost of solving for the mixing functions indicates that some optimum combination of the number channels and the number of trial functions exists, such that the most accurate and least expensive approximate solution results. The best combination will, of course, be problem dependent, and depend to a large extent on how clearly the choice of base functions is indicated for a particular problem. 
The Nature of the Synthesized Solution

Having obtained the approximate solution by the synthesis method, it is logical to ask in what sense does the approximate solution satisfy the system equations. This question may be answered by observing that Equation 2.16, in the example, could have been obtained by substituting the trial function $\phi$ into the system governing equation and requiring the integral of the residual error to vanish with respect to the weight functions $f_{j}^{*}(x)$. The approximate solution then satisfies the original equation, at every point along the remaining variables, in a weighted integral sense over the variables removed. Similarly, if the approximate adjoint solution were obtained by solving Equation 2.17, the resulting solution would satisfy the adjoint equation in a weighted Integral sense, the weight functions being the forward base functions $\mathbf{f}_{\mathbf{k}}(\mathrm{x})$.

In the multichannel procedure, the resulting approximate solution satisfies the system equation in a weighted integral sense over each of the channels, and can therefore be expected to more closely approximate the true solution. Indeed, as the number of channels is increased, the accuracy of the approximation can also be expected to increase.

The weighted residual nature of the synthesis solution suggests that the same approximation could be obtained from an application of the method of weighted residuals, without reference to the variational calculus. This is, in fact, the case since the indirect method of the variational calculus is a special case of the method of weighted. residuals. ${ }^{32}$ Utilization of the variational principle, however, allows in addition to the approximate solutions, a determination of the 
stationary value of the functional to a demonstrably high precision. Thus, if the functional is chosen such that its exact value is some Integral quantity of interest, then it may be approximated to "second order accuracy" when the trial functions are accurate to "first order". This is to say that if

$$
\Psi-\phi=O(\epsilon)
$$

and

$$
\Psi^{*}-\phi^{*}=O(\epsilon)
$$

then the value of the functional can be shown to be accurate to order $\epsilon^{2}, 1 . e \cdot$

$$
\mathscr{F}\left[\Psi, \Psi^{*}\right]-\mathscr{F}\left[\phi, \phi^{*}\right]=O\left(\epsilon^{2}\right)
$$

Although this fact has been the subject of some dispute in the literature, 39,40 Kaplan $^{41}$ has recently demonstrated that Equation 2.21 is indeed correct.

For linear, inhomogeneous equations of the form 2.1 , the adjoint source may be chosen as some reaction rate probability of interest, and the stationary value of the Roussopoulos functional is

$$
\tilde{K}\left[\Psi, \Psi^{*}\right]=\int \mathrm{dx} \int \mathrm{dy} \iint \mathrm{d} \hat{\Omega} \Sigma(\mathrm{x}, \mathrm{y}) \Psi(\mathrm{x}, \mathrm{y}, \hat{\Omega})
$$

This quantity may be approximated by the variational synthesis method with accuracy to second order. Note that this second order accuracy does not require that the solution for the adjoint mixing functions be obtained, but does require that the choice of adjoint base functions be 
made such that $x$ variation in the adjoint state function $\Psi^{*}$ is adequately represented. In this light, the variational synthesis method can be considered as a special case of the method of weighted residuals which specifies the choice of weight functions, 1.e., adjoint base functions, so as to give the best estimate of the quantity chosen as the stationary value of the functional.

Another great appeal of the variational synthesis method is the fact that the variational characterization of the system equations and their boundary and continuity conditions is a complete altermate description of the problem. In deriving approximate methods by the variational method, all assumptions regarding the approximation are embodied in the cholce of the form of the trial function. Once this choice is made, the approximate equations, their consistent boundary and continuity conditions, and consistent definitions of reduced system constants follow directly and unambiguously from the variational development. In obtaining approximations by simple application of the method of weighted residuals, the choice of consistent boundary and continuity conditions must be made aside from the development of the approximate equations themselves -- a fact which introduces the possibility of significant error. As a result of the completeness of the variational characterization, one finds in the literature examples where the redevelopment of classical approximations by the variational method has removed the ambiguity and arbitrariness in the choice of boundary and continuity conditions, 15,42 and has suggested alternate and possibly superior approaches to the approximation involved. 43 


\section{CHAPTER III}

\section{A VARIATIONAL SYNTHESIS APPROXIMATION FOR THE NEUTRON TRANSPORT PROBLEM}

The Boltzmann Transport Equation

The equation governing the behavior of the steady-state neutron distribution in the space-angle-energy phase space is the very general Boltzmann transport equation. It may be derived, assuming that statistical fluctuations about the average distribution are negligible, by requiring a particle balance within a differential volume element of phase space. Under the further assumptions that the presence of the neutrons does not alter the physical state of the system and that interactions between neutrons may be neglected, the equation becomes the linear Boltzmann equation and may be written as

$$
\begin{aligned}
& \hat{\Omega} \cdot \nabla \Psi(\vec{r}, \hat{\Omega}, E)+\Sigma_{t}(\vec{r}, E) \Psi(\vec{r}, \hat{\Omega}, E)=S(\vec{r}, \hat{\Omega}, E) \\
& +\iint_{4 \pi} \mathrm{d} \hat{\Omega}^{\prime} \int_{0}^{\infty} \mathrm{dE}^{\prime} \Sigma_{s}\left(\vec{r}, \hat{\Omega}, \hat{\Omega}^{\prime}, E, E^{\prime}\right) \Psi\left(\vec{r}, \hat{\Omega}^{\prime}, E^{\prime}\right)
\end{aligned}
$$

where

$$
\begin{aligned}
& \vec{r}=\text { the spatial position vector, } \\
& E=\text { the neutron energy, } \\
& \hat{\Omega}=\text { a unit vector in the direction of neutron motion, } \\
& \nabla=\text { the gradient operator, }
\end{aligned}
$$


$S(\vec{r}, \hat{\Omega}, E)=$ the source density, or rate per unit phase space volume at which neutrons are introduced into the system,

$\Psi(\vec{r}, \hat{\Omega}, E)=$ the neutron flux per unit energy and solid angle, $\Sigma_{t}(\vec{r}, E)=$ the macroscopic total interaction cross section at position $\vec{r}$ and energy $E$,

$\Sigma_{\mathbf{S}}\left(\vec{r}, \hat{\Omega}, \hat{\Omega}^{\prime}, E, E^{\prime}\right)=$ the macroscopic differential scattering cross section at position $\overrightarrow{\mathfrak{r}}$, for scattering events of neutrons in $\mathrm{dE} \mathrm{E}^{\prime}$ and $\mathrm{d} \hat{\Omega}^{\prime}$ which result in neutrons in $\mathrm{dE}$ and $\mathrm{d} \Omega$ per unit energy and solid angle.

Considering a volumetric region, $\mathfrak{R}$, enclosed by a convex surface, $s$, and composed of a finite number, $\Lambda$, of contiguous subregions $\Re_{\lambda}, \lambda=1,2, \ldots, \Lambda$, the boundary and continuity conditions necessary to complete the specification of the neutron distribution are:

a) Neutrons crossing the external surface are lost to the system, and

b) The neutron flux is spatially continuous across any internal interface between contiguous subregions.

These conditions may be expressed as

$$
\Psi(\vec{R}, \hat{\Omega}, \mathrm{E})=0, \hat{\Omega} \cdot \hat{\mathrm{s}}<0
$$

and

$$
\Psi\left(\vec{r}_{\lambda, \nu(\lambda)}, \hat{\Omega}, E\right)=\Psi\left(\vec{r}_{\nu(\lambda), \lambda}, \hat{\Omega}, E\right)
$$

with the notational conventions that: 


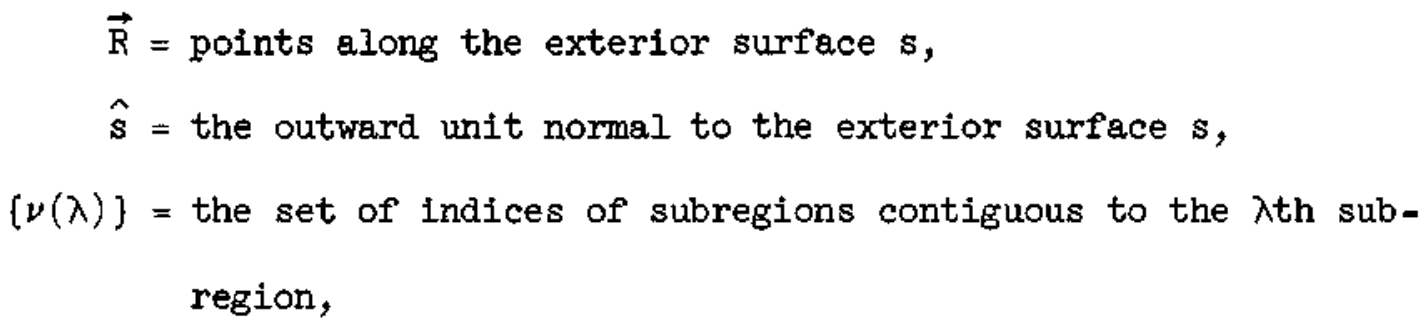

and

$\vec{r}_{\lambda ; \nu(\lambda)}=$ points along the interior surface between subregions $R_{\lambda}$ and $R_{\nu(\lambda)}$ as approached from within subregion $R_{\lambda} \cdot \vec{r}_{\nu(\lambda), \lambda}$ are the same points as approached within subregion ${ }^{R}(\lambda)$. With the further notational convention that $\sigma_{\lambda, \nu(\lambda)}=$ the interior surface between subregion $R_{\lambda}$ and $R_{\nu}(\lambda)$, $\hat{\sigma}_{\lambda}=$ the outward unit normal to interior surface of $R_{\lambda}$, $s_{\lambda}=$ that part of the exterior surface, $s$, belonging to subregion $R$,

and

$\mathrm{H}=$ the Heaviside step function defined such that

$$
H(t)=\left\{\begin{array}{l}
0, t<0 \\
I, t>0
\end{array}\right\}
$$

a complete variational characterization of the transport problem may be expressed as

$$
\delta \%=0
$$

where is the modified Roussopoulos functional: 


$$
\begin{aligned}
& \mathscr{Y}\left[\phi, \phi^{*}\right]=\sum_{\lambda=1}^{\Lambda}\left(\iiint_{\mathcal{R}} \mathrm{d} \vec{r} \iint_{4 \pi} \mathrm{d} \hat{\Omega} \int_{0}^{\infty} \mathrm{dE}\left\{\phi^{*}[S-\mathrm{T} \phi]+\mathrm{S}^{*} \phi\right\}\right. \\
& +\sum_{\nu(\lambda) \sigma_{\lambda, \nu(\lambda)}} \iint_{4 \pi} d \sigma \iint_{\pi} d \hat{\Omega} \int_{0}^{\infty} \mathrm{dE}\left\{\mathrm { H } ( - \hat { \Omega } \cdot \sigma _ { \lambda } ) ( \hat { \Omega } \cdot \sigma _ { \lambda } ) \phi ^ { * } ( \vec { r } _ { \lambda , \nu ( \lambda ) } , \hat { \Omega } , E ) \left[\phi\left(\vec{r}_{\lambda, \nu(\lambda)}, \hat{\Omega}, E\right)\right.\right. \\
& \left.\left.-\phi\left(\vec{r}_{\nu(\lambda), \lambda}, \hat{\Omega}, E\right)\right]\right\}+\iint_{s_{\lambda}} d s \iint_{4 \pi} d \hat{\Omega} \int_{0}^{\infty} d E\left\{H\left(-\hat{\Omega} \cdot \hat{s}(\hat{\Omega} \cdot \hat{s})\left(\phi^{*}(\vec{R}, \hat{\Omega}, E) \phi(\vec{R}, \hat{\Omega}, E)\right\}\right)\right.
\end{aligned}
$$

In this expression, the first integral corresponds to the transport equation itself, where $T(\vec{r}, \hat{\Omega}, E)$ is the linear transport operator and the arguments of the functions in the integral are assumed understood. The second integral accounts for the continuity of the angular flux at the interfaces between contiguous subregions and the third term corresponds to the external vacuum boundary condition. Thus constructed, the functional admlts as arguments functions $\phi$ and $\phi^{*}$ which are piecewise continuous functions of the variables $\vec{r}, \hat{\Omega}$ and $E$, and. which do not satisfy the boundary conditions imposed on the true forward and adjoint angular fluxes $\Psi$ and $\Psi *$. As shown in the development of this functional in Appendix A, the vanishing of the variational derivation of $F$ with respect to $\phi^{*}$ yields, as Euler equations deter mining the function $\phi(r, \hat{\Omega}, E)$, the Boltzmann Iquation 3.1 and the conditions of Equations 3.2 and 3.3. Vanishing of the variational derivative with respect to $\phi$ then yields the adjoint Boltzmann equation and its consistent boundary and continuity conditions. Thus, witrin 
the very general class of piecewise continuous functions of $\vec{r}, \hat{\Omega}$ and $E$, the modified Roussopoulos functional as given by Equation 3.6 , has, as Its stationary functions, the continuous solutions to the forward and adjoint Boltzmann equations.

\section{The Energy Approximation}

The Multigroup Energy Approximation

In this approximation the energy variable will be treated in the standard multigroup energy method. This approximation, developed originally from physical arguments, segments the continuous energy range into energy groups and determines the energy dependence of the neutron distribution in a groupwise manner. The following redevelopment will serve to illustrate how historical approximations, their consistent boundary and continuity conditions, and consistent definitions of the approximate system constants may be obtained clearly and systematically by use of the variational method.

Given an energy spectrum, $X(E)$, which will serve to approximate the energy dependence of the angular neutron flux density $\Psi(\vec{r}, \hat{\Omega}, E)$, and an associated adjoint energy spectrum, $X^{*}(E)$, to approximate the energy dependence of the adjoint flux density $\Psi^{*}(\vec{r}, \hat{\Omega}, E)$, trial functions for the approximate forward and adjoint fluxes are formed as

$$
\phi(\overrightarrow{\mathbf{r}}, \hat{\Omega}, \mathrm{E})=\sum_{\mathrm{g}=1}^{\mathrm{G}} \phi_{\mathrm{g}}(\overrightarrow{\mathrm{r}}, \hat{\Omega}) \Delta_{\mathrm{g}} X(\mathrm{E})
$$

and 


$$
\phi^{*}(\vec{r}, \hat{\Omega}, E)=\sum_{g=I}^{G} \phi_{g}^{*}(\vec{r}, \hat{\Omega}) \Delta_{g} X^{*}(E)
$$

Here $G$ is the total number of energy groups or segments into which the continuous energy range is divided and $\Delta_{\mathrm{g}}$ is a step function which is unity within the gth energy segment and zero elsewhere. Denoting by $\mathrm{E}_{\mathrm{g}}$ the upper energy of the gth segment and adopting the standard notation that group numbers increase with decreasing energy, the functions $\triangle_{\mathrm{g}}$ may be expressed as

$$
\Delta_{g}=\left\{\begin{array}{ll}
1, & E_{g+I}<E<E_{g} \\
0, & \text { otherwise }
\end{array}\right\}
$$

The energy spectra $X(E)$ and $X^{*}(E)$ are the forward and adjoint base function for this approximation and the unknown mixing functions $\phi_{g}$ and $\phi_{\mathrm{g}}^{*}$ will be determined by imposing the variational principle on the reduced functional.

Inserting the trial functions 3.7 and 3.8 into the functional of Equation 3.6 and integrating over energy yields the reduced functional

$$
\begin{gathered}
\mathscr{F}_{\mathrm{g}}\left[\phi_{\mathrm{g}}, \phi_{\mathrm{g}}^{*}\right]=\sum_{\mathrm{g}=1}^{\mathrm{G}} \mathrm{w}_{\mathrm{g}} \sum_{\lambda=1}^{\Lambda}\left(\int \int \int _ { \mathcal { R } _ { \lambda } } d \vec { \mathrm { r } } \int \int _ { 4 \pi } d \hat { \Omega } \left\{\phi_{\mathrm{g}}^{*}(\overrightarrow{\mathrm{r}}, \hat{\Omega})\left[\mathrm{s}_{\mathrm{g}}(\overrightarrow{\mathrm{r}}, \hat{\Omega})-\mathrm{T}_{\mathrm{g}}(\overrightarrow{\mathrm{r}}, \hat{\Omega}) \phi_{\mathrm{g}}(\overrightarrow{\mathrm{r}}, \hat{\Omega})\right]\right.\right. \\
\left.+\mathrm{s}_{\mathrm{g}}^{*}(\overrightarrow{\mathrm{r}}, \hat{\Omega}) \phi_{\mathrm{g}}(\overrightarrow{\mathrm{r}}, \hat{\Omega})\right\}
\end{gathered}
$$




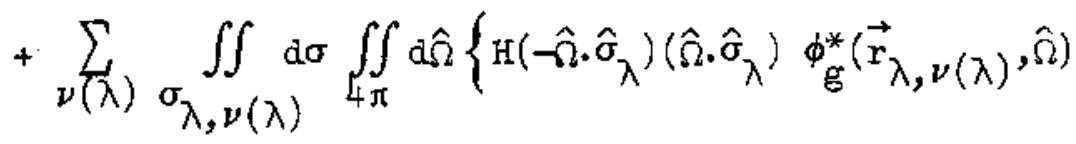

$$
\begin{aligned}
& \left.\times\left[\phi_{\mathrm{g}}\left(\overrightarrow{\mathrm{r}}_{\lambda, \nu(\lambda)}, \hat{\Omega}\right)-\phi_{\mathrm{g}}\left(\overrightarrow{\mathrm{r}}_{\nu}(\lambda), \lambda, \hat{\Omega}\right)\right]\right\} \\
& \left.+\iint_{\hat{s}} \mathrm{ds} \iint_{4 \pi} \mathrm{d} \hat{\Omega}\left\{\mathrm{H}(-\hat{\Omega} \cdot \hat{\mathrm{s}})(\hat{\Omega} \cdot \hat{\mathrm{s}}) \phi_{\mathrm{g}}^{*}(\vec{R}, \hat{\Omega}) \phi_{g}(\vec{R}, \hat{\Omega})\right\}\right)
\end{aligned}
$$

This is the multigroup functional where

$$
\begin{gathered}
w_{g}=\int_{E_{g+1}}^{E} x^{*}(E) \chi(E) d E \\
S_{g}(\vec{r}, \hat{\Omega})=\frac{1}{w_{g}} \int_{E}^{E} \chi_{g+1}^{E}(E) S(\vec{r}, \hat{\Omega}, E) d E \\
S_{g}^{*}(\vec{r}, \hat{\Omega})=\frac{1}{w_{g}} \int_{E g+1}^{E} S_{g *(\vec{r}, \hat{\Omega}, E) X(E) d E}
\end{gathered}
$$

and $\mathrm{T}_{\mathrm{g}}(\vec{r}, \hat{\Omega})$ is the multigroup form of the linear transport operator.

Imposing the variational principle on the multigroup functional by requiring it to be stationary with respect to arbitrary variations in the adjoint mixing functions $\phi_{\mathrm{g}}^{*}$ results in a set of equations which must be satisfied by the $\phi_{\mathrm{g}}$. Writing the multigroup transport operator $\mathrm{T}_{\mathrm{g}}(\vec{r}, \hat{\Omega})$ explicitly these equations become 


$$
\begin{gathered}
\hat{\Omega} \cdot \nabla \phi_{g}(\vec{r}, \hat{\Omega})+\Sigma_{t_{g}}(\vec{r}) \phi_{g}(\vec{r}, \hat{\Omega}) \\
=S_{g}(\vec{r}, \hat{\Omega}) \frac{1}{w_{g}} \sum_{g^{\prime}=1}^{G} \iint_{\pi} d \hat{\Omega}^{\prime} \Sigma_{s_{g g^{\prime}}}\left(\vec{r}, \hat{\Omega}, \hat{\Omega}^{\prime}\right) \phi_{g^{\prime}}\left(\vec{r}, \hat{\Omega}^{\prime}\right) w_{g^{\prime}} \\
\phi_{g}(\vec{R}, \hat{\Omega})=0, \hat{\Omega} \cdot \hat{s}<0 \\
\phi_{g}\left(\vec{r}_{\lambda, \nu(\lambda)}, \hat{\Omega}\right)=\phi_{g}\left(\vec{r}_{\nu(\lambda), \lambda}, \hat{\Omega}\right)
\end{gathered}
$$

The consistent definitions of the multigroup total and scattering cross sections resulting from the variational development are

$$
\Sigma_{t g}(\vec{r})=\frac{1}{w_{g}} \int_{E_{g+1}}^{E} X^{*}(E) \Sigma_{t}(\vec{r}, E) X(E) d E
$$

and

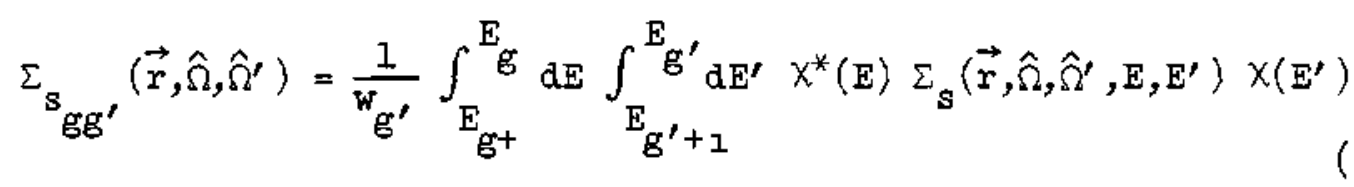

Recognizing Equation 3.14 as the multigroup form of the transport equation, the mixing functions $\phi_{g}(\vec{r}, \hat{\Omega})$ can be interpreted physically as the average angular neutron flux per unit energy over energy group g. Equations 3.15 and 3.16 are, respectively, the consistent multigroup boundary condition requiring no incoming neutrons through the external surface $s$ and the continuity condition requiring spatial continuity of the multigroup angular fluxes for each energy group. 
Historically the multigroup equations have been written in terms of the group integrated flux rather than the group average flux. By renormalizing the energy spectrum functions $X^{*}(E)$ and $X(E)$ such that

$$
\int_{E_{g+1}}^{E_{g}} x^{*}(E) d E=1
$$

and

$$
\int_{E_{g+1}}^{E_{g}} \chi^{*}(E) \chi(E) d E=w_{g}=1
$$

Equation 3.14 takes on the classical multigroup form and the mixing functions $\phi_{\mathrm{g}}(\vec{r}, \hat{\Omega})$ may be interpreted as the usual multigroup angular flux.

Requiring the multigroup functional to be stationary with respect to arbitrary variations in the $\phi_{\mathrm{g}}$ yields equations determining the $\phi_{g}^{*}$, which are recognized as the multigroup adjoint transport equation and consistent boundary and continuity conditions. The mixing functions $\phi_{\mathrm{g}}^{*}(\vec{r}, \hat{\Omega})$ may thus be interpreted as the usual multigroup adjoint angular flux.

Other Energy Approximations. Other approximate treatments of the continuous energy variable utilized in the reactor field may also be derived by the variational method, and could have been chosen for the method being developed in this work. For the sake of generality, the trial function choice leading to several of the more common treatments will be discussed.

The overlapping group, or spectral synthesis, method may be obtained by utilizing a set of energy base functions $X_{k}(E)$ and $\chi_{k}^{*}(E)$, 
$k=1,2, \ldots, k$ and forming the trial functions

$$
\phi(\vec{r}, \hat{\Omega}, E)=\sum_{k=1}^{K} \phi_{k}(\vec{r}, \hat{\Omega}) x_{k}(E)
$$

and

$$
\phi *(\vec{r}, \hat{\Omega}, E)=\sum_{j=1}^{K} \phi(\vec{j}, \vec{r}, \hat{\Omega}) x_{j}^{*}(E)
$$

The usual reduction procedure then yields equations determining the mixing functions $\phi_{k}(\vec{r}, \hat{\Omega})$. One notes here that no groupwise energy segmentation has been utilized, since the step functions $\Delta_{g}$ have not been included in the trial functions, and as a result the reduced total cross section $\Sigma_{t}$ will be a full square matrix of order $K$. The multigroup total cross section, when written as a square matrix of order G, is a diagonal matrix as a result of the use of the step functions $\Delta_{g}$. The multigroup energy approximation with regionwise spectrum averaged constants may be developed by utilizing a forward and adjoint energy spectrum, $x_{\lambda}(E)$ and $x_{\lambda}^{*}(E)$, for each of the subregions $R_{\lambda}$. The trial functions are then

$$
\phi(\vec{r}, \hat{\Omega}, E)=\sum_{\lambda=1}^{\Lambda} \sum_{g=1}^{G} \phi_{g}(\vec{r}, \hat{\Omega}) \Delta_{g} x_{\lambda}(E) \Delta_{\lambda}
$$

and

$$
\phi^{*}(\overrightarrow{\mathbf{r}}, \hat{\Omega}, E)=\sum_{\lambda=1}^{\Lambda} \sum_{\mathrm{g}=1}^{G} \phi_{\mathrm{g}}^{*}(\overrightarrow{\mathbf{r}}, \hat{\Omega}) \Delta_{g} \chi_{\lambda}^{*}(E) \Delta_{\lambda}
$$

where $\Delta_{\lambda}$ is a step function of position, $\vec{r}$, which is unity the subregion $R_{\lambda}$ and zero over all other subregions. Equations resulting for 
the mixing functions $\phi_{\mathrm{g}}(\vec{r}, \hat{\Omega})$ in this approximation will be the same as Equations 3.14 through 3.16 if each of the pairs of base functions $x_{\lambda}(E)$ and $x_{\lambda}^{*}(E)$ are normalized according to Equations 3.19 and 3.20 . The multigroup constants appearing in these equations will then be reglonwise dependent in that they were obtained by averaging over regionwise dependent spectra $X_{\lambda}(E)$ and $X_{\lambda}^{*}(E)$, using the formulas in Equations 3.17 and 3.18 .

The multigroup lethargy approximation is obtained in a manner analogous to the multigroup energy approximation by introducing the change of variable in the functional

$$
u=\ln \left(\frac{E_{O}^{\prime}}{E}\right)
$$

where $u$ is the lethargy variable and $E_{0}$ is an arbitrarily chosen energy at which the neutron lethargy is defined to be zero. Choosing the spectrum functions $X(u)$ and $x^{*}(u)$, the trial functions are written as

$$
\phi(\vec{r}, \hat{\Omega}, \mathrm{u})=\sum_{\mathrm{g}=1}^{\mathrm{G}} \phi_{\mathrm{g}}(\overrightarrow{\mathrm{r}}, \hat{\Omega}) \Delta_{\mathrm{g}} \mathrm{x}(\mathrm{u})
$$

and

$$
\phi^{*}(\overrightarrow{\mathbf{r}}, \hat{\Omega}, \mathrm{u})=\sum_{\mathbf{g}=1}^{\mathrm{G}} \phi_{\mathrm{g}}^{*}(\overrightarrow{\mathrm{r}}, \hat{\Omega}) \Delta_{\mathrm{g}} x^{*}(\mathrm{u})
$$

The function $\Delta_{g}$ must now be defined as

$$
\Delta_{g}=\left\{\begin{array}{ll}
1 & u_{g+1} \leqslant u<u_{g} \\
0 & \text { otherwise }
\end{array}\right\} ; g=1,2, \ldots, g
$$


where $u_{g}$ is the upper lethargy boundary of group $g$. With no normalization of the spectrum functions, the mixing functions $\phi_{\mathrm{g}}(\overrightarrow{\mathbf{r}}, \hat{\Omega})$ and $\phi_{\mathrm{G}}^{*}(\vec{r}, \hat{\Omega})$ may be interpreted as the average angular fluxes per unit lethargy over lethargy group $g$.

\section{The Spatial Synthesis Approximation}

The variational synthesis method will now be applied to the multigroup transport problem to effectively remove one or more of the spatial variables from consideration. In that the gradient term in the transport operator is dependent upon the coordinate system chosen, only the case of rectangular geometry will be considered here. If, for the case of three-dimensional systems, two-dimensional transport solutions for several related problems may be obtained, then the method to be developed will specify a manner in which these two-dimensional solutions may be combined to form an approximate three-dimensional solution. Similarly, for the case of two-dimensional systems, the method will specify the manner in which solutions to related one-dimensional problems may be combined to obtain approximate two-dimensional solutions.

This approximation will be developed in detail for the twodimensional case, since the geometric and notational complexity is much less than for the three-dimensional case. The development for the three-dimensional case follows precisely along the same lines, yielding reduced equations very similar to those of the two-dimensional case. The equations for the three-dimensional case will be given in Appendix B. 
The Two-Dimensional Case

Consider a typical rectangular two-dimensional reactor as shown in Figure 1. The reactor is assumed to be composed of a number of rectangular subregions, as indicated, over which the macroscopic material properties are constent. Placement of the rectangular subregions is seen to be such that a natural segmentation of the $\mathrm{y}$-axis is indicated. These segments along the y-axis are termed "axial zones", and as seen in the figure, there is no y-variation of the macroscopic properties within an axial zone. Notice, however, that within an axial zone the macroscopic properties do vary with $\mathrm{x}$ in a plecewise constant manner.

To construct an approximate two-dimensional solution to the transport equation by the variational synthesis method, base functions $f_{k}(x)$ and $f_{j}^{*}(x)$, which are representative of the $x$-variation of the true forward and adjoint solutions, must be obtained by solving related one-dimensional problems. The natural segmentation of the $y$-axis into axial zones suggests that if a one-dimensional calculation along the $\mathrm{x}$-axis were performed for each axial zone considexed separately, the resultant $x$-variations of the one-dimensional angular fluxes could be combined to give a good approximation to the $x$-variation of the true two-dimensional solution.

Noting that if the number of axial zones, $M$, is large the total number of base functions obtained in this manner may be quite large, it is advantageous to allow the trial functions to be discontinuous. In doing so, the base functions from the one-dimensional calculation of a particular zone may be used in the trial function only at points 


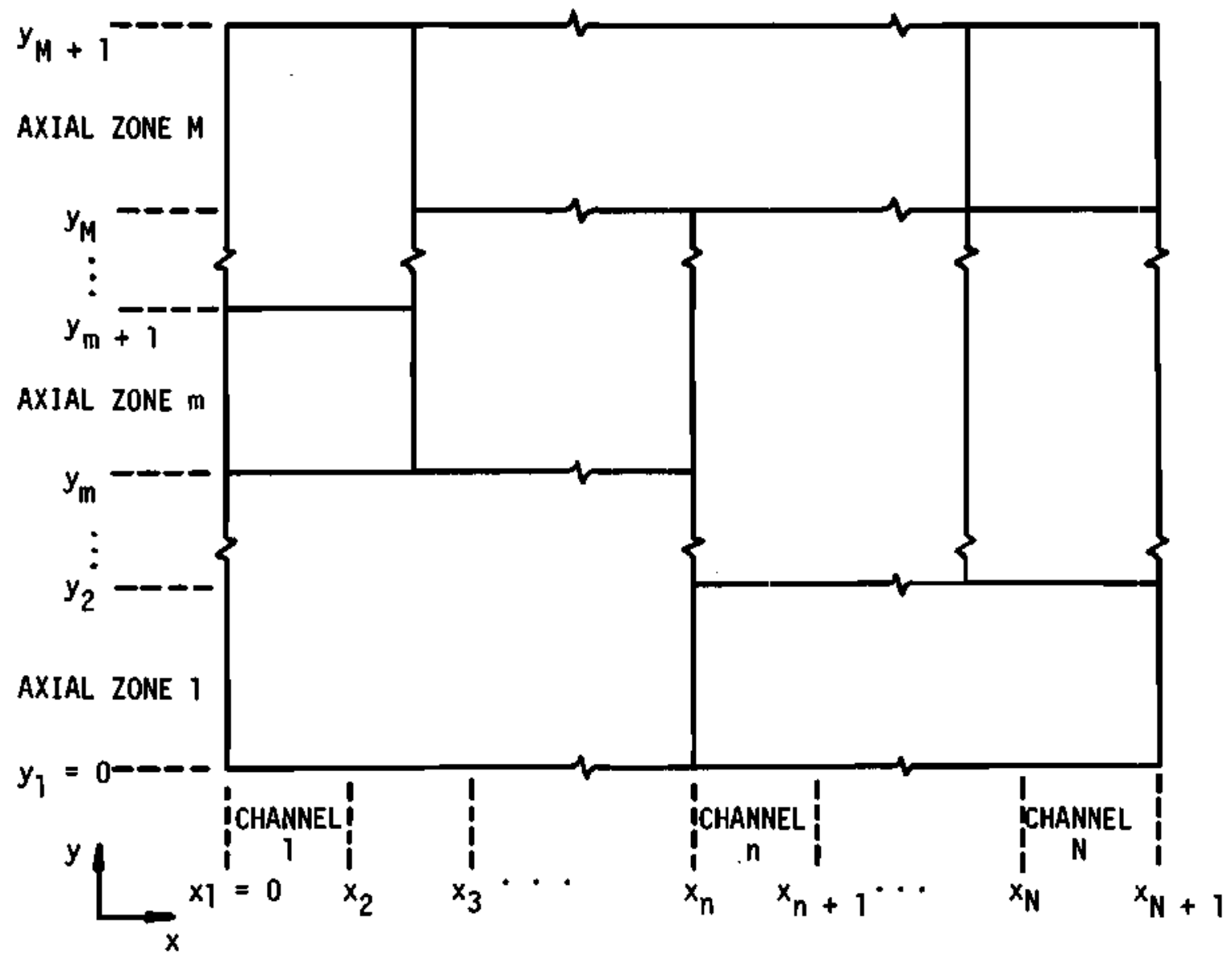

Figure 1. Geometry for Typical Two-Dimensional Rectangular Reactor 
on the $y$-axis within and near that particular zone. Then, for extmple, base functions obtained from one-dimensional calculations of axial zones near the top of the reactor and which may bear little resemblance to the true solution near the bottom of the reactor, need not be j.ncluded in the trial function in axial zones near the bottom. Anticipating more difficult situations where the choice of the base function is not so clearly defined, the base function axis, $x$, is segmented into channels. Such situations might arise, for example, where base functions from two adjacent axial zones are radically clifferent or where the heights of several adjacent axial zones are so small that the $x$-variation of the true solution is not characteristic only of those zones.

One further observation about the nature of the transport problem may be effectively incorporated into the choice of the form of the trial function. This is the observation of the similarity of the $x-$ variation of the angular flux for all angles with $\Omega_{x}<0$, where $\Omega_{x}$ is the $x$-direction cosine, and the similarity of the $x$-variation for all angles with $\Omega_{x}>0$. There is, however, little similarity between the $x$-variations of the angular fluxes belonging to different ranges of the $x$-direction cosine. Since the base functions from the one-dimensional calculations also share this characteristic, it is advantageous to utilize the base functions only over the range of $\Omega_{x}$ where they are, in fact, most representative.

With the above considerations, the trial functions for this approximation have been chosen for the forward multigroup angular flux as 


$$
\begin{aligned}
\phi_{\mathrm{g}}(\mathrm{x}, \mathrm{y}, \hat{\Omega}) & =\sum_{\mathrm{n}=1}^{\mathrm{N}} \Delta_{\mathrm{n}} \sum_{\mathrm{k}=1}^{\mathrm{K}} \mathrm{H}\left(\Omega_{\mathrm{x}}\right) \phi_{\mathrm{gnk}}^{-}(\mathrm{y}, \hat{\Omega}) \mathrm{f}_{\mathrm{gk}(\mathrm{m})}^{-}(\mathrm{x}) \\
& +\mathrm{H}\left(\Omega_{\mathrm{x}}\right) \phi_{\mathrm{gnk}}^{+}(\mathrm{y}, \hat{\Omega}) \mathrm{f}_{\mathrm{gk}(\mathrm{m})}^{+}(\mathrm{x})
\end{aligned}
$$

and for the adjoint, as

$$
\begin{aligned}
\phi_{\mathrm{g}}^{*}(\mathrm{x}, \mathrm{y}, \hat{\Omega}) & =\sum_{\mathrm{n}=1}^{\mathrm{N}} \Delta_{\mathrm{n}} \sum_{\hat{j}=1}^{\mathrm{K}} \mathrm{H}\left(-\Omega_{\mathrm{x}}\right) \phi_{\mathrm{gn} \bar{n}_{j}}^{*}(\mathrm{y}, \hat{\Omega}) \mathrm{f}_{\mathrm{gj}}^{*-}(\mathrm{m}) \\
& +\mathrm{H}\left(\Omega_{\mathrm{x}}\right) \phi_{\mathrm{gnj}}^{*+}(\mathrm{y}, \hat{\Omega}) \mathrm{f}_{\mathrm{gj}(\mathrm{m})}^{*+}(\mathrm{x})
\end{aligned}
$$

where $\mathrm{g}$ is the energy group index, $\mathrm{n}$ is the channel index, and $\mathrm{j}$ and $k$ are the indices for the forward and adjoint base functions respectively. The index $m$ refers to the axial zone and the notation $j(n)$ and $k(m)$ is used to indicate that the base functions may be different In different axial zones. In this expression the step functions $\Delta_{n}$ and $H$ are as previously defined, and the mixing functions and base functions now have the superscript + or - to indicate the range of $\Omega_{x}$ which they represent.

By the usual procedure, the trial functions of Equations 3.29 and 3.30 are substituted into the multigroup functional of Equation 3.10 , and the integration over the base function variable, $x$, performed. The presence of the step functions $H\left(\Omega_{x}\right)$ and $H\left(\Omega_{X}\right)$ effectively splits the angular integral in the functional into two parts and, using matrix notation, the reduced multigroup functional becomes 


$$
\begin{aligned}
& F_{[}\left[\phi_{g n}^{ \pm}, \phi_{g n}^{* \pm}\right]=\sum_{g=1}^{G} \sum_{n=1}^{N} \sum_{m=1}^{M}\left(\int _ { y _ { m } } ^ { y _ { m + 1 } } d y \int \int _ { 4 \pi } d \hat { \Omega } \left\{\sum _ { \alpha = + , - } H ( \alpha \Omega _ { x } ) \left[\phi_{g n}^{* \alpha}\left(S_{g n m}^{\alpha}-T_{g n m}^{\alpha} \phi_{g n}^{\alpha}\right)\right.\right.\right. \\
& \left.\left.+\mathrm{s}_{g n \mathrm{~m}}^{* \alpha} \phi_{\mathrm{gn}}^{\alpha}\right]\right\} \\
& +\int_{y_{m}}^{y_{I n+1}} d y \iint_{4 \pi} d \hat{\Omega}\left\{H ( \Omega _ { x } ) \phi _ { g n } ^ { * + } ( y , \hat { \Omega } ) \Omega _ { x } C _ { g n m } ^ { + } \left\langle\left(1-\delta_{n l}\right)\left[\phi_{g n-1}^{+}(y, \hat{\Omega})-\phi_{g n}^{+}(y, \hat{\Omega})\right]\right.\right. \\
& \left.+\delta_{\mathrm{n} 1} \phi_{\mathrm{gn}}^{+}(\mathrm{y}, \hat{\Omega})\right\rangle \\
& \left.+H\left(-\Omega_{x}\right) \phi_{g n}^{*}(y, \hat{\Omega}) \Omega_{x} C_{g n+1 m}^{-}\left\langle\left(1-\delta_{n N}\right)\left[\phi_{g n}^{-}(y, \hat{\Omega})-\phi_{g n+1}^{-}(y, \hat{\Omega})\right]+\delta_{n N^{\prime n}} \phi_{g}^{-}(y, \hat{\Omega})\right\rangle\right\} . \\
& +\iint_{4 \pi} \mathrm{d} \hat{\Omega}\left\{\mathrm { H } ( \Omega _ { \mathrm { y } } ) \sum _ { \alpha = + , - } \mathrm { H } ( \alpha \Omega _ { \mathrm { x } } ) \Omega _ { \mathrm { y } } \phi _ { \mathrm { gn } } ^ { * \alpha } ( \mathrm { y } _ { \mathrm { m } , \mathrm { m } - 1 } , \hat { \Omega } ) \left[\mathrm{D}_{\mathrm{gnmm-1}}^{\alpha} \phi_{\mathrm{gn}}^{\alpha}\left(\mathrm{y}_{\mathrm{m}-1, \mathrm{~m}}, \hat{\Omega}\right)\right.\right. \\
& \left.-A_{\mathrm{gnm}}^{\alpha} \phi_{\mathrm{gn}}^{\alpha}\left(\mathrm{y}_{\mathrm{m}, \mathrm{m}-1}, \hat{\Omega}\right)\right] \\
& +\mathrm{H}\left(\Omega_{\mathrm{y}}\right) \sum_{\alpha=+,-} \mathrm{H}\left(\alpha \Omega_{\mathrm{x}}\right) \Omega_{\mathrm{y}} \phi_{\mathrm{gn}}^{* \alpha}\left(\mathrm{y}_{\mathrm{m}, \mathrm{m}+1}, \hat{\Omega}\right)\left[\mathrm{A}_{\mathrm{gnm}}^{\alpha} \phi_{\mathrm{gn}}^{\alpha}\left(\mathrm{y}_{\mathrm{m}, \mathrm{m}+1}, \hat{\Omega}\right)\right. \\
& \left.\left.-D_{g n m+1}^{\alpha} \phi_{g n}^{\alpha}\left(y_{m+1, m}, \hat{\Omega}\right)\right]\right\} \\
& +\iint_{4 \pi} \mathrm{d} \hat{\Omega} \sum_{\alpha=+,-} \mathrm{H}\left(\alpha \Omega_{\mathrm{x}}\right) \Omega_{\mathrm{y}}\left[\delta_{\mathrm{mi}} \mathrm{H}\left(\Omega_{\mathrm{y}}\right) \phi_{\mathrm{gn}}^{* \alpha}\left(\mathrm{y}_{\mathrm{m}}, \hat{\Omega}\right){ }_{\mathrm{Amm}}^{\alpha} \phi_{\mathrm{gn}}^{\alpha}\left(\mathrm{y}_{\mathrm{m}}, \hat{\Omega}\right)\right. \\
& \left.\left.+\delta_{m M} H\left(-\gamma_{y}\right) \phi_{g n}^{* \alpha}\left(y_{m+1}, \hat{\Omega}\right) A_{g n m}^{\alpha} \phi_{g n}^{\alpha}\left(y_{m+1}, \hat{\Omega}\right)\right]\right)
\end{aligned}
$$

Here the reduced transport operators $\mathrm{T}_{\mathrm{gnm}}^{+}(\mathrm{y}, \hat{\Omega})$ and $\mathrm{T}_{\mathrm{gnm}}^{-}(\mathrm{y}, \hat{\Omega})$ are defined, in matrix form, such that 
39

$$
\begin{aligned}
T_{\mathrm{gnm}}^{\alpha}(\mathrm{y}, \hat{\Omega}) \phi_{\mathrm{gn}}^{\alpha}(\mathrm{y}, \hat{\Omega})=\Omega_{\mathrm{x}} \mathrm{B}_{\mathrm{gnm}}^{\alpha} \phi_{\mathrm{gn}}^{\alpha}(\mathrm{y}, \hat{\Omega})+\Omega_{\mathrm{y}} \mathrm{A}_{\mathrm{gnm}}^{\alpha} \frac{\partial}{\partial \mathrm{y}} \phi_{\mathrm{gn}}^{\alpha}(\mathrm{y}, \hat{\Omega})+\Sigma_{\mathrm{tgnm}}^{\alpha} \phi_{\mathrm{gn}}^{\alpha}(\mathrm{y}, \hat{\Omega}) \\
\quad-\sum_{\mathrm{g}^{\prime}=1}^{\mathrm{G}} \iint_{4 \pi} \mathrm{d} \hat{\Omega}^{\prime}\left[\mathrm{H}\left(-\Omega_{\mathrm{x}}^{\prime}\right) \Sigma_{\mathrm{sgg^{ \prime } \mathrm { nm }}}^{\alpha-}\left(\hat{\Omega}, \hat{\Omega}^{\prime}\right) \phi_{\mathrm{g}^{\prime} \mathrm{n}}^{-}\left(\mathrm{y}, \hat{\Omega}^{\prime}\right)\right. \\
\left.+\mathrm{H}\left(\Omega_{\mathrm{x}}^{\prime}\right) \Sigma_{\mathrm{sgg^{ \prime } \mathrm { nm }}}^{\alpha+}\left(\hat{\Omega}, \hat{\Omega}^{\prime}\right) \phi_{\mathrm{g}^{\prime} \mathrm{n}}^{+}\left(\mathrm{y}, \hat{\Omega}^{\prime}\right)\right] ; \alpha=+,-
\end{aligned}
$$

and the reduced constants are matrices whose $j, \mathrm{kth}$ elements are defined consistently as

$$
\begin{gathered}
A_{g n m}^{\alpha j k}=\int_{x_{n}}^{x_{n+1}} f_{g j(m)}^{* \alpha}(x) f_{g k(m)}^{\alpha}(x) d x ; \alpha=+,- \\
B_{g n m}^{\alpha j k}=\int_{x_{n}}^{x_{n+1}} f_{g j(m)}^{* \alpha} \frac{d}{d x} f_{g k(m)}^{\alpha}(x) d x ; \alpha=+,- \\
C_{g n m}^{\alpha j k}=f_{g j(m)}^{* \alpha}\left(x_{n}\right) f_{g k(m)}^{\alpha}\left(x_{n}\right) ; \alpha=+,-
\end{gathered}
$$

$$
\begin{aligned}
& D_{g n m m^{\prime}}^{\alpha j k}=\int_{x_{n}}^{x_{n+1}} f_{g j(m)}^{* \alpha}(x) f_{g k\left(m^{\prime}\right)}^{\alpha}(x) d x ; \alpha=+,-; m^{\prime}=m-1, m+1 \\
& \Sigma_{t g n m}^{\alpha j k}=\int_{x_{n}}^{x_{n+1}} f_{g j(m)}^{* \alpha}(x) \Sigma_{t g m}(x) f_{g k(m)}^{\alpha}(x) d x ; \alpha=+,-
\end{aligned}
$$

and

$$
\Sigma_{s g g^{\prime} n m}^{\alpha \beta j k}=\int_{x_{n}}^{x_{n+1}} f_{g j(m)}^{* \alpha}(x) \Sigma_{s g g^{\prime} m}\left(x, \hat{\Omega}, \hat{\Omega}^{\prime}\right) f_{g^{\prime} k(m)}^{\beta}(x) d x ; \alpha, \beta=+,-
$$


The unknowns $\phi_{\mathrm{gn}}^{ \pm}$and $\phi_{\mathrm{gn}}^{* \pm}$ are, of course, column vectors whose elements are the mixing functions $\phi_{\text {gnk }}^{ \pm}$and $\phi_{\text {gnj }}^{* \pm}$, and the forward and adjoint sources are column vectors whose elements are

$$
S_{g n m}^{\alpha j}(\hat{\Omega})=\int_{x_{n}}^{x} n+1 f_{g j(m)}^{* \alpha}(x) s_{g m}(x, \hat{\Omega}) d x ; \alpha=+,-
$$

and

$$
S_{g n m}^{* \alpha k}(\hat{\Omega})=\int_{x_{n}}^{x} S_{g m}^{*}(x, \hat{\Omega}) f_{g k(m)}^{\alpha}(x) d x ; \alpha=+,
$$

Note that the $\mathrm{y}$-dependence in the reduced constants and sources appears only as an index, $\mathrm{m}$, since over each axial zone these have been assumed constant. In the above expression for the reduced functional, transposition of the adjoint source and mixing function vectors necessary for proper matrix multiplication is assumed understood, as is the fact that the matrices $\mathrm{D}_{\text {gnmm }}^{ \pm}$are the null matrices for $\mathrm{m}^{\prime}<0$ and $\mathrm{m}^{\prime}>\mathrm{M}$. The notation $\mathrm{y}_{\mathrm{m}, \mathrm{m}-1}$ and $\mathrm{y}_{\mathrm{m}-1, \mathrm{~m}}$ is used to indicate the value of $\mathrm{y}_{\mathrm{m}}$ as approached from within axial zone $\mathrm{m}$ and $\mathrm{m}-1$ respectively, and the symbol $\delta$ is the Kronecker delta.

The equations determining the forward mixing functions $\phi_{\mathrm{gn}}^{+}(\mathrm{y}, \hat{\Omega})$ and $\phi_{\mathrm{gn}}^{-}(\mathrm{y}, \hat{\Omega})$ are found in the usual way by requiring the reduced f'unctional to be stationary with respect to arbitrary variations in the $\phi_{\mathrm{gn}}^{*+}(\mathrm{y}, \hat{\Omega})$ and $\phi_{\mathrm{gn}}^{*}(\mathrm{y}, \hat{\Omega})$. In order to solve the resulting equations, however, some angular approximation must be employed. This angular approximation may be obtained consistently from the variational method by choosing an appropriate trial function to approximate the angular dependent mixing functions $\phi_{\mathrm{gn}}^{ \pm}(\mathrm{y}, \hat{\Omega})$ and $\phi_{\mathrm{gn}}^{* \pm}(\mathrm{y}, \hat{\Omega})$. 
The angular approximation chosen here is the discrete ordinates approximation, which segments the continuous angular variable in a manner analogous to the multigroup energy segmentation. To accomplish this, the unit sphere is divided into D discrete segments and the angular step functions, $\Delta_{d}$, are defined such that

$$
\Delta_{d}=\left\{\begin{array}{ll}
1 & \text { for } \hat{\Omega} \text { within segment } \mathrm{d} \\
0 & \text { otherwise }
\end{array}\right\} \mathrm{d}=1,2, \ldots, D
$$

One restriction to be imposed on this segmentation 1s that, within each octant of the unit sphere, there must be an integral number of discrete segments.

The use of the Heaviside step functions $H\left(\Omega_{x}\right)$ and $H\left(\Omega_{x}\right)$ iri the multigroup trial function of Equation 3.29 has, in the reduced problem, divided the angular dependence into two ranges, $\Omega_{x}<0$ and $\Omega_{x}>0$. Without any loss of generality, the angular segments, $d=1,2, \ldots, D$, in tke discrete ordinates approximation may be ordered such that the segments, $d=1,2, \ldots, \frac{D}{2}$, lie in the range $\Omega_{x}<0$ and the segments, $d=\frac{D}{2}+1, \ldots, D$, lie in the range $\Omega_{x}>0$. With this convention, the trial functions for the discrete ordinates approximation to the angular variable may be written as

$$
\begin{aligned}
\cdot \phi_{\mathrm{gn}}^{-}(\mathrm{y}, \hat{\Omega}) & =\sum_{\mathrm{d}=1}^{\mathrm{D} / 2} \phi_{\mathrm{gnd}}(\mathrm{y}) \Delta_{\mathrm{d}} \\
\phi_{\mathrm{gn}}^{+}(\mathrm{y}, \hat{\Omega}) & =\sum_{\mathrm{d}=\frac{\mathrm{D}}{2}+{ }_{\mathrm{gnd}}}^{\mathrm{D}}(\mathrm{y}) \Delta_{\mathrm{d}}
\end{aligned}
$$




$$
\begin{gathered}
\phi_{\mathrm{gn}}^{*}-(\mathrm{y}, \hat{\Omega})=\sum_{\mathrm{d}=1}^{\mathrm{D} / 2} \phi_{\text {gnd }}(\mathrm{y}) \Delta_{\mathrm{d}} \\
\phi_{\mathrm{gn}}^{*+}(\mathrm{y}, \hat{\Omega})=\sum_{\mathrm{d}=\frac{\mathrm{D}}{2}+1}^{\mathrm{D}} \phi_{\text {gnd }}(\mathrm{y}) \Delta_{\mathrm{d}}
\end{gathered}
$$

These are matrix equations implying that the same expansion be applied to each element in the column matrices. The discrete mixing functions $\phi_{\text {gnd }}$ and $\phi_{\text {gnd }}^{*}$ are thus column vectors, each containing $K$ elements to be denoted as $\phi_{\text {gndk }}(y)$ and $\phi_{\text {gnd }}^{*}(y)$. Note that the superscript + or is not required on the discrete mixing functions since the range of $\Omega_{x}$ for each discrete segment may be inferred from the value of the subscript d.

Now, upon substitution of the trial functions into the reduced functional of Equation 3.31, the angular integration may be performed to obtain a further reduced functional depending only on the unknown discrete mixing functions $\phi_{\mathrm{gnd}}(\mathrm{y})$ and $\phi_{\mathrm{gnd}}^{*}(\mathrm{y})$. The requirement that this reduced functional be stationary with respect to arbitrary variations in the $\phi_{\text {gnd }}^{*}(y)$ then yields the following equations and conditions which must be satisfied by the $\phi_{\text {gnd }}(y)$ :

$$
\eta_{\mathrm{d}} \mathrm{A}_{\mathrm{gnm},}^{-} \frac{\mathrm{d}}{\mathrm{dy}} \phi_{\mathrm{gnd}}(\mathrm{y})+\left[\Sigma_{\text {tgnm }}^{-}+\mu_{\mathrm{d}_{\mathrm{gnm}}}\right]_{\mathrm{gnd}}^{-}(\mathrm{y})=\mathrm{s}_{\mathrm{gnmd}}^{-}
$$

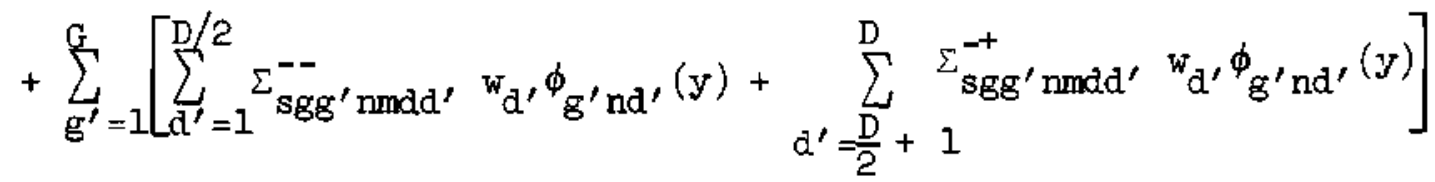

$$
+\mu_{d} C_{g n+1 m}^{-}\left\{\left(1-\delta_{n N}\right)\left[\phi_{g n d}(y)-\phi_{g n+1 d}(y)\right]+\delta_{n N^{g n d}}(y)\right\} ; \mu_{d}<0(3.46 a)
$$


43

$$
\begin{aligned}
& \eta_{d} A_{\text {gnm }}^{+} \frac{d}{d y} \phi_{\text {gnd }}(y)+\left[\Sigma_{\text {tgnm }}^{+}+\mu_{d} B_{\text {gnm }}^{+}\right] \phi_{\text {gnd }}(y)=s_{\text {gnmd }}^{+} \\
& +\sum_{g^{\prime}=1}^{G}\left[\sum_{d^{\prime}=1}^{D / 2} \Sigma^{+-} \mathrm{sgg}^{\prime} n m d d^{\prime} w_{d^{\prime}} \phi_{g^{\prime} n d^{\prime}}(y)+\sum_{d^{\prime}=\frac{D}{2}+1}^{D} \Sigma_{s g g^{\prime} n m d d^{\prime}}^{++} w_{d^{\prime}} \phi_{g^{\prime} n d^{\prime}}(y)\right] \\
& +\mu_{\mathrm{d}} \mathrm{C}_{\mathrm{gnm}}^{+}\left\{\left(1-\delta_{\mathrm{nl}}\right)\left[\phi_{\mathrm{gn}-1 \mathrm{~d}}(\mathrm{y})-\phi_{\mathrm{gnd}}(\mathrm{y})\right]-\varepsilon_{\mathrm{nl}} \phi_{\mathrm{gnd}}(\mathrm{y})\right\} ; \mu_{\mathrm{d}}>0 \quad(3.46 \mathrm{~b}) \\
& \mathrm{D}_{\mathrm{gnmm-1}}^{-} \phi_{\text {gnd }}\left(\mathrm{y}_{\mathrm{m}-1, \mathrm{~m}}\right)=\mathrm{A}_{\text {gnm }}^{-} \phi_{\text {gnd }}\left(\mathrm{y}_{\mathrm{m}, \mathrm{m}-1}\right) ; \mu_{\mathrm{d}}<0, \eta_{\mathrm{d}}>0 \\
& A_{\text {gnm }}^{-} \phi_{\text {gnd }}\left(y_{m, m+1}\right)=D_{\text {gnmm+1 }}^{-} \phi_{\text {gnd }}\left(y_{m+1, m}\right) ; \mu_{d}<0, \eta_{d}<0 \\
& \mathrm{D}_{\text {gnmm-1 }}^{+} \phi_{\text {gnd }}\left(\mathrm{y}_{\mathrm{m}-1, \mathrm{~m}}\right)=\mathrm{A}_{\mathrm{gnm}}^{+} \phi_{\text {gnd }}\left(\mathrm{y}_{\mathrm{m}, \mathrm{m}-1}\right) ; \mu_{\mathrm{d}}>0, \eta_{\mathrm{d}}>0 \\
& A_{\text {gnm }}^{+} \phi_{\text {gnd }}\left(y_{m, m+1}\right)=D_{\text {gnnm+1 }}^{+} \phi_{\text {gnd }}\left(y_{m+1, m}\right) ; \mu_{d}>0, \eta_{d}<0 \\
& \phi_{\text {gnd }}\left(y_{1}\right)=0 ; \eta_{d}>0 \\
& \left.\phi_{\text {gnd }}\left(\mathrm{y}_{\mathrm{M}+1}\right)=0 ; \eta_{\mathrm{d}}<\right)
\end{aligned}
$$

The discrete angular constants appearing in these equations are defined consistently as

$$
\begin{gathered}
w_{d}=\iint_{4 \pi} \Delta_{d} d \hat{\Omega} \\
\mu_{d}=\frac{1}{w_{d}} \iint_{4 \pi} \Omega_{x} \Delta_{d} d \hat{\Omega}
\end{gathered}
$$




$$
\eta_{\mathrm{d}}=\frac{1}{\mathrm{w}_{\mathrm{d}}} \iint_{4 \pi} \Omega_{\mathrm{y}} \Delta_{\mathrm{d}} \mathrm{d} \hat{\widehat{\Omega}}
$$

and may be interpreted as the number of steradians subtended by discrete segment $d$ and the average values of the direction cosines $\Omega_{k}$ and $\Omega_{\mathrm{y}}$ in segment $\mathrm{d}$, respectively. The discrete angular sources and scattering cross sections are now given by

$$
\begin{aligned}
& S_{\text {gnmd }}^{\alpha}=\frac{1}{w_{d}} \iint_{4 \pi} s_{\text {gnm }}^{\alpha}(\hat{\Omega}) \Delta_{d} d \hat{\Omega} ; \alpha=+,- \\
& S_{\text {gnmd }}^{* \alpha}=\frac{1}{w_{d}} \iint_{4 \pi} s_{g n m}^{* \alpha}(\hat{\Omega}) \Delta_{d} d \hat{\Omega} ; \alpha=+,-
\end{aligned}
$$

and

$$
\Sigma_{s g g^{\prime} n m d d^{\prime}}^{\alpha \beta}=\frac{1}{w_{d^{w} d^{\prime}}} \iint_{4} \mathrm{~d} \hat{\Omega} \iint_{4 \pi} \mathrm{d} \hat{\Omega}^{\prime} \Delta_{d^{2}} \Sigma_{s g g^{\prime} n m}^{\alpha \beta}\left(\hat{\Omega}, \hat{\Omega}^{\prime}\right) \Delta_{d^{\prime}} ; \alpha, \beta=+,-
$$

Equations 3.46 completely determine the unknown discrete angular mixing functions, and must be solved to obtain an approximate two-dimensional multigroup transport solution by the variational synthesis method. Several observations pertinent to these equations may now be made.

The form of Equations $3.46 \mathrm{a}$ and $3.46 \mathrm{~b}$ is seen to be similar to the direct one-dimensional discrete-ordinates equations with an angular dependent buckling term and an added source term due to coupling between the channels. This similarly suggests that the standard finitedifference treatment successful in the direct discrete-ordinates solutions would be applicable to the remaining independent variable, $\mathrm{y}$, in 
these equations. Also, the angular dependence in these equations is seen to be represented by two discrete direction cosines, $\mu_{d}$ and $\eta_{d}$. The direct one-dimensional discrete-ordinates equations differ in that symmetry arguments allow the angular variable to be represented by only one discrete direction cosine.

The nature of the added source term due to channel coupling: has strong physical appeal since each channel is coupled to the neighboring channels in a directional sense; i.e., channel $n$ is coupled to channel $\mathrm{n}+1$ through neutrons with $\mu_{d}<0$ and to channel $\mathrm{n}-1$ through neutrons with $\mu_{d}>0$. The same directional sense is evident in the coupling between axial zones as prescribed by the continuity conditions $3.46 \mathrm{c}$ through $3.46 \mathrm{f}$.

Now examine the consequences of the choice of the trial function utilizing different base functions in the positive and negative ranges of $\Omega_{x}$. The matrix constants $A_{g n m}^{+}$and $A_{\text {gnm }}^{-}$are each square matrices of order $K$, as are the matrix constants $B_{g n m}^{ \pm}, C_{g n m}^{ \pm}$, $D_{g n m m^{\prime}}^{ \pm}$and $\Sigma_{\text {tgnm }}^{ \pm}$, and the discrete mixing functions $\phi_{\text {gnd }}(y)$ are each $K$ by 1 column vectors. The number of elements associated with each pair of matrices $\left(+\right.$ and - ) is this $2 K^{2}$. If all the base functions had been used in the expansion in both ranges of $\Omega_{\mathrm{X}}$, the matrices $A_{\mathrm{gnm}}, \mathrm{B}_{\mathrm{gnm}}, \mathrm{C}_{\mathrm{gnm}}, \mathrm{D}_{\mathrm{gnnm}}$, and $\Sigma_{\text {tgnm }}$ would each be square matrices of order $2 \mathrm{~K}$ containing $4 \mathrm{~K}^{2}$ elements, and the mixing functions $\phi_{\text {gnd }}(y)$ would be $2 K$ by 1 column vectors. By utilizing the trial functions of Equations 3.29 and 3.30 , the number of matrix elements to be calculated has been significantly reduced and the number of unknown mixing functions has been halved. In that the difficulty of solving for the mixing functions varies 
roughly as the square of the number unknowns, the total cost of the approximate solution has been significantly reduced by this dual range segmentation of $\Omega_{x}$.

In that the weighted scattering cross section serves to couple the two reduced Equations $3.46 \mathrm{a}$ and $3.46 \mathrm{~b}$, no reduction in its size has been accomplished by the half range segmentation of $\Omega_{x}$. The size of the matrix may be reduced by representing the anisotropic scattering as the first few terms in a Legendre series expansion, as is stanclard practice in direct calculations. In this manner the double angular subscript $d, d^{\prime}$ mey be replaced by a single index $l$, denoting the lth term in the Legendre series. In many cases the inclusion of only two terms in the series is adequate, and the transport approximation may be used requiring only the scattering matrix $\Sigma_{s g^{\prime}}^{\alpha \beta}$, with the total cross section being replaced by the transport cross section. It should be mentioned at this point that if the discrete angular approximation had been made prior to the spatial synthesis approximation in this development, the double angular subscripts $d$ and $d^{\prime}$ could not be removed in this manner. The Three-Dimensional Case

To utilize the method to obtain approximate three-dimensional solutions, related two-dimensional problems would be solved to obtain multigroup base functions of two spatial variables, $f_{g k}(x, y)$ and $\mathrm{f}_{\mathrm{g} j}^{*}(\mathrm{x}, \mathrm{y})$, and the variational synthesis method used to obtain reduced equations for the mixing functions dependent on the remaining variables $z$ and $\hat{\Omega}$. The three-dimensional reactor will be assumed to be composed of rectangular subregions, placed so as to indicate a natural coarise 
mesh segmentation of the $z$ axis into axial zones over which no $z$ variation in macroscopic properties occurs. As in the two-dimensional case, this axial zone segmentation of the remaining spatial variable, $z$, will yield the obvious suggestion as to which related two-dimensional problems should be solved to obtain the base functions. The segmentation of the base function axes, $x$ and $y$, is accomplished by defining segments $n=1,2, \ldots, N$ along the $x$-axis and $\mathrm{m}=1,2, \ldots, \mathrm{M}$ alones the $\mathrm{y}$-axis. The term channel will then be applied to each portion of the $\mathrm{x}-\mathrm{y}$ plane possessing unique subscripts $\mathrm{n}$, $\mathrm{m}$, as formed by the $x$ and $y$ segmentation. A segmentation of the angular range, analogous to the half-range segmentation of $\Omega_{x}$ in the two-dimensional problem, is the division into four ranges defined by the combinations of positive and negative ranges of $\Omega_{x}$ and $\Omega_{y}$.

The trial function for the approximate three-dimensional solution is thus formed, for each energy groups, as

$$
\begin{aligned}
& \phi_{g}(x, y, z, \hat{\Omega})=\sum_{n=1}^{N} \Delta_{n} \sum_{m=1}^{M} \Delta_{m} \sum_{k=1}^{K}\left\{H\left(\Omega_{x}\right) H\left(\Omega_{y}\right) \phi_{g n m k}^{I}(z, \hat{\Omega}) f_{g k(l)}^{I}(x, y)\right.
\end{aligned}
$$

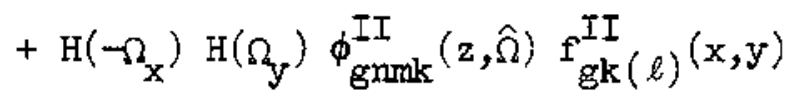

$$
\begin{aligned}
& +H\left(\Omega_{x}\right) H\left(\Omega_{y}\right) \phi_{\text {gnmk }}^{I I I}(z, \hat{\Omega}) \operatorname{fig}_{\mathrm{gk}(\ell)}^{I I I}(x, y) \\
& \left.+H\left(\Omega_{x}\right) H\left(\Omega_{y}\right) \phi_{g n m k}^{I V}(z, \hat{\Omega}) f_{g k(\ell)}^{I V}(x, y)\right\}
\end{aligned}
$$

The same form for the adjoint trial function is used, in terms of the adjoint base functions. The superscripts I, II, III and IV are used here to denote the following ranges of $\Omega_{x}$ and $\Omega_{y}$, respectively, 
I: $\Omega_{\mathrm{x}}>0, \Omega_{\mathrm{y}}>0$

II: $\Omega_{\mathrm{x}}<0, \Omega_{\mathrm{y}}>0$

III: $\Omega_{\mathrm{x}}<0, \Omega_{\mathrm{y}}<0$

IV: $\Omega_{\mathrm{x}}>0, \Omega_{\mathrm{y}}<0$

and serve to replace the superscripts + and - used in the two-dimensional case. These superseripts then denote the ranges of the variables $\Omega_{x}$ and $\Omega_{y}$ in which the base functions are to be used.

Now, by the same two step reduction procedure used previously, the base function variables are removed and the angular dependence of the mixing functions treated by the discrete-ordinates approximation. This procedure then results in equations determining the discrete angular mixing functions, $\phi_{\text {gnmd }}(z)$, and their consistent boundary and continuity conditions. By ordering the angular segments, $d=1,2, \ldots, D$, such that the first $\frac{D}{4}$ lie in the angular region $I$, and the next three sequences of $\frac{D}{4}$ segments lie in the regions II, III, and IV, as given above, the equations and conditions determining the discrete angular mixing functions are formally similar to those for the two-dimensional case. These equations and the corresponding definitions of the reduced constants will be given in Appendix B.

\section{Method of Solution}

The application of a straightforward central differencing technique to the remaining spatial variable in Equations $3.46 \mathrm{a}$ and $3.46 \mathrm{~b}$, or to the corresponding equations for the three-dimensional case, allows the determination of the discrete angular mixing function along that variable. The method of solution to be presented here is 
applicable to both cases, but will be developed particularly using the notation for the two-dimensional problem.

To obtain the finite-difference form, define a spatial mesh along the $\mathrm{y}$-axis with increments, $I=1,2, \ldots$, IMAX, such that over each axial zone, $\mathrm{m}$, the widths of the increments are constant and given by $h_{m}$. Denoting by $\phi_{\text {gndI }}$ the value of the discrete angular mixing functions at the center of increment $I$, and $\phi_{\text {gnd } 1}$ as the value at the lower edge of increment I, the derivative over increment I in Equations $3.46 \mathrm{a}$ and $3.46 \mathrm{~b}$ may be represented as

$$
\frac{\mathrm{d}}{\mathrm{dy}} \phi_{\text {gnd }}(\mathrm{y}) \cong \frac{\phi_{\text {gndi+1 }}-\phi_{\text {gndi }}}{\mathrm{h}_{\mathrm{m}}}
$$

for each increment in axial zone $m$. Further, the assumption of a linear variation over each mesh increment allows the centered values $\phi_{\text {gnaI }}$ to be expressed in terms of the edge values as

$$
\phi_{\text {gndI }}=\frac{1}{2}\left(\phi_{\text {gndi }}+\phi_{\text {gndi }+1}\right)
$$

Inserting these expressions into Equations $3.46 \mathrm{a}$ and $3.46 \mathrm{~b}$ then yields the finite difference form amenable to computer solution. For the most efficient solution it is advantageous to adopt the convention. that the discrete angular segmentation be symmetric with respect to 180 degree rotation, then each quadrant of the unit sphere contains $\frac{D}{4}$ discrete segments and for every segment with discrete cosine $\eta_{d}$ there is also a segment with the cosine $-\eta_{d}$. Without loss of generality, the discrete elements are further ordered such that for the discrete segment, $d$, having cosines $\mu_{d}, \eta_{d},\left(\eta_{d}>0\right)$, the discrete segment $d^{\prime}$ with 
cosines $\mu_{\mathrm{d}^{\prime}}=\mu_{\mathrm{d}}$ and $\eta_{\mathrm{d}^{\prime}}=-\eta_{\mathrm{d}}$ is the segment $\mathrm{d}^{\prime}=\mathrm{d}+\frac{\mathrm{D}}{4}$. With this convention, one solves the finite difference equations for $\phi_{\text {gndi+ }}$ in terms of $\phi_{\text {gndi }}$ for discrete angles with $\eta_{\mathrm{d}}>0$ and for $\phi_{\text {gndi }}$ in terms of $\phi_{\text {gndi+1 }}$ for angles with $\eta_{d}<0$. The I mesh is then swept by channel from $\mathrm{n}=\mathrm{l}$ to $\mathrm{n}=\mathrm{N}$ for discrete angles with $\mu_{\mathrm{d}}>0$ and from $\mathrm{n}=\mathrm{N}$ to $\mathrm{n}=1$ for discrete angles with $\mu_{d}<0$ for each energy group. The equations to be solved for each group at each point on the mesh, and the order of their solution, thus become

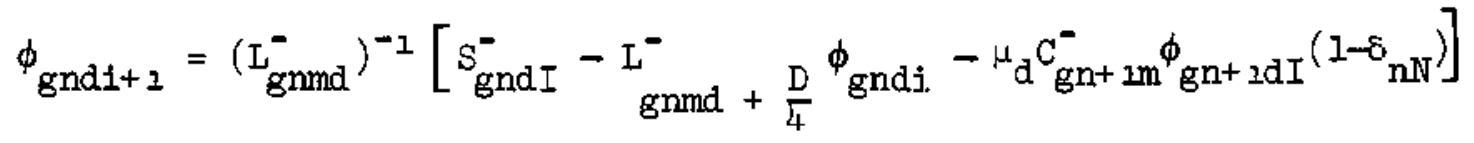

$$
\begin{aligned}
& \mathrm{d}=1,2, \ldots, \frac{\mathrm{D}}{4} ; \mathrm{n}=1,2, \ldots, \mathrm{N} ; \mathrm{i}=1,2, \ldots, \mathrm{IMAX} \\
& \phi_{\text {gnd 1+ 1 }}=\left(I_{\text {gnmd }}^{+}\right)^{-1}\left[s_{\text {gndI }}^{+}-I_{\text {gnmd }}^{+}+\frac{D}{4} \phi_{\text {gndi. }}+\mu_{d} c_{\text {gnm }}^{+} \phi_{\text {gn-1dI }}\left(1-\delta_{n j}\right)\right] \\
& \mathrm{d}=\frac{\mathrm{D}}{2}+1, \ldots, \frac{3 \mathrm{D}}{4} ; \mathrm{n}=\mathrm{N}, \mathrm{N}-1, \ldots, 1 ; \mathrm{i}=1,2, \ldots, \text { IMAX } \\
& \phi_{\text {gndi }}=\left(L_{\text {gnmd }}^{-}\right)^{-1}\left[S_{\text {gndI }}^{-}-I_{\text {gnmd }}^{-}-\frac{D}{4} \phi_{\text {gndi+1 }}-\mu_{d} C_{\text {gn+m }}^{-} \phi_{\text {gn+ } 1 d I}\left(1-\delta_{n N}\right)\right] \\
& d=\frac{D}{4}+1, \ldots, \frac{D}{2} ; n=1,2, \ldots, N ; i=\operatorname{IMAX}, \operatorname{IMAX}-1, \ldots, 1 \quad(3.56 \mathrm{c}) \\
& \phi_{\text {gndi }}=\left(\mathrm{I}_{\text {gnmd }}^{+}\right)^{-1}\left[\mathrm{~S}_{\text {gndI }}^{+}-\mathrm{L}_{\text {gnmd }}^{+}-\frac{\mathrm{D}}{4} \phi_{\text {gndi+1 }}+\mu_{\mathrm{d}} \mathrm{C}_{\text {gnm }}^{+} \phi_{\text {gn-1dI }}\left(1-\delta_{\mathrm{nI}}\right)\right] \\
& \mathrm{d}=\frac{3 \mathrm{D}}{4}+1, \ldots, \mathrm{D} ; \mathrm{n}=\mathrm{N}, \mathrm{N}-1, \ldots, \mathrm{1} ; \mathrm{i}=\operatorname{IMAX}, \operatorname{IMAX}-1, \ldots, 1 \quad(3.56 \mathrm{~d})
\end{aligned}
$$$$
\text { In these equations } S_{\text {gndI }}^{ \pm} \text {represents the total source including scatter- }
$$$$
\text { ing and the matrices } \mathrm{L}_{\text {gnmd }}^{ \pm} \text {are defined as }
$$ 


$$
\begin{aligned}
& L_{\text {gnmd }}^{+}=\frac{\mu_{d} B_{g n m}^{+}}{2}+\frac{\eta_{d} A_{g n m}^{+}}{h_{m}}+\frac{\Sigma_{\text {tgnm }}^{+}}{2}+\frac{\mu_{d} C_{g n m}^{+}}{2} \\
& L_{\text {gnmd }}^{-}=\frac{\mu_{d} B_{g n m}^{-}}{2}+\frac{\eta_{d} A_{g n m}^{-}}{h_{m}}+\frac{\Sigma_{\text {tgnm }}^{-}}{2}-\frac{\mu_{d} C_{g n+1 m}^{-}}{2}
\end{aligned}
$$

Note that the discrete segments are now ordered such that for angular segments $d$, with $\eta_{d}>0$,

$$
\mathrm{L}_{\text {gnmd }}^{\alpha}=\mathrm{L}_{\text {grmd }+\frac{\mathrm{D}}{4}}^{\alpha} ; \alpha=+,-
$$

and for segments $d$ with $\eta_{d}<0$,

$$
\mathrm{L}_{\text {gnmd }}^{\alpha}=\mathrm{L}_{\text {gnmd }}^{\alpha}-\frac{\mathrm{D}}{4} ; \alpha t=+,-
$$

so that only $\mathrm{D} / 2$ matrices for each group, channel and axial zone need be calculated and stored to obtain the approximate solution. The Iteration Procedure

Since the right hand sides of Equations 3.56a through $3.56 \mathrm{cl}$ depend on the unknowns $\phi_{\text {gndi }}$ through the scattering source term, some iteration procedure must be employed to effect the solution. The source term in these equations may be considered to be composed of three distinct parts: the contribution from the fixed source, the contribution from the scattering of neutrons into group g from al]. other groups $\mathrm{g}^{\prime}$, and the contribution from the scattering of neutrons within group g. Over most of the energy range of interest in neutron transport problems, neutrons undergoing scattering collisions are degraded in energy. Thus, if the solution is begun with the highest 
energy group and then obtained for each energy group, g, by proceeding downward in energy, the contribution to the source from scattering in higher energy groups is completely determined. Then the complete solution for a particular energy group, g, may be represented formally as

$$
\tilde{\phi}_{g}=\mathscr{L}_{\mathrm{g}}\left(\widetilde{S}_{\mathrm{g}}+\tilde{\Sigma}_{\mathrm{sg}} \tilde{\phi}_{\mathrm{g}}\right)
$$

where $\tilde{\phi}_{g}$ is the total solution for the discrete angular mixing functions in energy group $g . \mathcal{L}_{\mathrm{g}}$ is the inverse of the finite difference form of the complete reduced transport operator, which operates on the total source to group $\mathrm{g}$ and yields the solution for $\tilde{\phi}_{\mathrm{g}} \cdot \tilde{\mathrm{S}}_{\mathrm{g}}$ is the flxed source to group glus the contribution from scattering in other groups $\mathrm{g}^{\prime} \cdot \tilde{\Sigma}_{\mathrm{sg}}$ is the within-group scattering operator which operates on the discrete angular mixing functions $\tilde{\phi}_{\mathrm{g}}$ to yield the third contribution to the total source to group $g$.

As the solution proceeds through the energy groups from upper to lower energies, an iterative technique must be applied at each energy group to obtain its solution. These groupwise iterations are termed "inner iterations", and the most straightforward technique to employ is the standard Gaussian or power iteration method. 44 This procedure is begun by assuming a first guess to the solution, $\boldsymbol{\phi}_{\mathrm{g}}^{\circ}$, which leads to the first iterate solution

$$
\tilde{\phi}_{g}^{3}=\mathscr{L}_{g}\left(\tilde{S}_{g}+\tilde{\Sigma}_{s g} \tilde{\phi}_{g}^{o}\right)
$$

The first iterate solution them becomes the next guess and the procedure repeated 


$$
\begin{gathered}
\tilde{\phi}_{\mathrm{g}}^{2}=\mathcal{L}_{\mathrm{g}}\left(\tilde{\mathrm{S}}_{\mathrm{g}}+\tilde{\Sigma}_{\mathrm{sg}} \tilde{\phi}_{\mathrm{g}}^{: L}\right) \\
\vdots \\
\tilde{\phi}_{\mathrm{g}}^{\mathrm{t}}=\mathcal{L}_{\mathrm{g}}\left(\tilde{\mathrm{S}}_{\mathrm{g}}+\tilde{\Sigma}_{\mathrm{sg}} \tilde{\phi}_{\mathrm{g}}^{\mathrm{t}-1}\right)
\end{gathered}
$$

Defining the absolute error vector after $t$ iterations as

$$
\xi^{t}=\tilde{\phi}_{g}-\delta_{g}^{t}
$$

where $\tilde{\phi}_{\mathrm{g}}$ is the solution to Equation 3.61 and $\tilde{\phi}^{\mathrm{t}}$ is given by Equation 3.636 , it follows by direct substitution that

$$
\tilde{g}^{t}=\left(\mathcal{L}_{g} \tilde{\Sigma}_{s g}\right)^{t} \epsilon_{g}^{o}
$$

The inner iteration procedure for each energy group should then ccntinue through as many iterations as are required to reduce the absolute error for that group, $\varepsilon_{g}^{t}$, to an acceptably small level. The absolute error $\delta_{g}^{t}$ cannot be calculated during the iterative procedure, however, since it depends on the unknown solution $\tilde{\phi}_{g}$ and a relative error vector for each inner iteration must be used. The elements of this relative error vector may be defined in terms of the iterate solutions $t$ and $t-1$ as

$$
\epsilon_{g}^{t}=\left|\frac{\phi_{g}^{t}}{\phi_{g}^{t-1}}-1\right|
$$

where each element of the error vector is given by this equation in terms of the corresponding elements of the iterate solution vectors. 
An indication that the iterative process is convergent is that

$$
\epsilon_{g}^{t}<\epsilon_{g}^{t-1}
$$

and a useful measure by which to terminate the procedure is

$$
\max \left(\epsilon_{g}^{t}\right) \leqslant \gamma
$$

where $\max \left(\epsilon_{\mathrm{g}}^{t}\right)$ is the maximum relative error in the elements of the solution vector, and $\gamma$ is the chosen acceptable error, usually $10^{-3}$ or less. 45

Other inner iteration methods could also be employed, as are discussed by Mynatt ${ }^{44}$ in similar context, and several techniques may be applied to accelerate the convergence of the iterative process. One useful and straightforward technique by which to accelerate the convergence of the Gaussian iteration procedure is the method of over.. relazation. This procedure seeks to drive the iterative solution toward the converged solution by enhancing the calculated relative change between successive iterations. It may be applied to the iterative solution vector, $\hat{\phi}_{g}^{t}$, itself, or alternatively it may be applj.ed to the iterative values of the within-group scattering source. Thus, if the value of the within-group scattering contribution to the total source after iteration $t-1$ is given by

$$
\tilde{\Sigma}_{s g} \phi_{g}^{t-1}
$$

then the value after the next iteration, denoted now by, 


$$
\left(\tilde{\Sigma}_{S g} \oint_{g}^{t}\right)^{*}
$$

is, for a convergent process, closer to the true value of that cortribution. Then the change in value

$$
\left(\tilde{\Sigma}_{S g} \oint_{g}^{t}\right)^{*}-\tilde{\Sigma}_{S g} \oint_{g}^{t-1}
$$

represents a step in the right direction (i.e., toward the converged value), and the effectiveness of this step may be enhanced by driving the value in this direction using the relation

$$
\tilde{\Sigma}_{s g} \phi_{g}^{t}=\tilde{\Sigma}_{s g} \oint_{g}^{t-1}+\beta\left[\left(\tilde{\Sigma}_{s g} \hat{\phi}_{g}^{t}\right)^{*}-\tilde{\Sigma}_{s g} \oint_{g}^{t-I}\right]
$$

Here $\beta$ is the overrelaxation, or driving, factor and the driven value of the within-group scattering source,

$$
\tilde{\Sigma}_{s g} \tilde{\phi}_{8}^{t}
$$

replaces the starred value as the value after iteration $t$.

The optimum value of the overrelaxation factor, $\beta$, is dependent upon the nature of the combined operator, $\mathcal{L}_{\mathrm{g}} \tilde{\Sigma}_{\mathrm{sg}}$, but can be determined only for certain simplified cases. In practice, experience has shown that values in the range

$$
1.0 \leqslant \beta \leqslant 1.6
$$

may be used effectively. For the value of

$$
\beta=1.0
$$


it will be noted from Equation 3.69, that the change is not driver. at all, and

$$
\tilde{\Sigma}_{\text {sg }} \tilde{\phi}_{\mathrm{g}}^{\mathrm{t}}=\left(\tilde{\Sigma}_{\mathrm{sg}} \tilde{\phi}_{\mathrm{g}}^{\mathrm{t}}\right)^{*}
$$

During the first few iterations of the process, large changes in the values of the within-group scattering contributions usually occur, and care must be taken that the values not be driven too far by the overrelaxation process. To avoid such complications due to large changes in first few iterations, the overrelaxation process is constrained, as advocated by Vondy, ${ }^{45}$ by limiting the driven values to the range

$$
0.5\left(\tilde{\Sigma}_{s g} \tilde{\phi}_{g}^{t}\right)^{*} \leqslant \tilde{\Sigma}_{s g} \tilde{\phi}_{g}^{t} \leqslant\left[\left(\tilde{\Sigma}_{s g} \tilde{\phi}_{g}^{t}\right)^{*}+\tilde{\Sigma}_{s g} \tilde{\phi}_{g}^{t-1}\right]
$$

If all scattering events result in a degradation of neutron energy, as is the case with fast reactors, then the solution may be obtained from a single pass through all the groups, solving by inner iteration for each group vector, $\tilde{\phi}_{g}$, as described above. For thermal reactor problems, however, where neutrons may gain energy from a scattering event, the solution for some group g may depend on the solution for some lower energy group, $g^{\prime}$, which is not yet determiried when the group $g$ solution is found. In such cases it is necessary to perform "outer iterations", by passing through all the energy groups repeatedly until the relative error between passes has been reduced. to an acceptable level. The solution guess to be utilized at the beginning of each pass through the groups is, of course, the solution from the previous outer iteration. 
Summary of the Approximation Procedure

The procedure to be utilized in obtaining approximate twodimensional solutions to the neutron transport equation by the variational synthesis approximation developed in this chapter may be summarized into the following steps:

1. Examine the geometry of the reactor to be calculated, and in particular, observe the natural segmentation of the spatial variable which is not removed.

2. Perform calculations with computer codes utilizing the direct solution methods for one-dimensional problems related to the problem at hand.

3. Using the solutions to the lower dimensional problems as the base functions, calculate the synthesis matrix constants as given by Equations 3.33 through 3.38 and 3.50 through 3.52 , and utilize these to calculate the finite-difference matrix L as given by Equations 3.57 and 3.58

4. Solve Equations 3.56 a through 3.56 d for the discrete ansular mixing coefficients.

5. Using the discrete angular mixing coefficients, determine the approximate solution by recombination with the base functions using the trial function expression. The approximate multigroup angular flux for all angles within discrete segment d, obtained by combination of the spatial and angular trial functions of Equations 3.29 , 3.42 and 3.43 , is then 


$$
\begin{aligned}
& \phi_{g d}(x, y, \hat{\Omega})=\sum_{n=1}^{N} \Delta_{n} \sum_{k=1}^{K} \phi_{g n d k}(y) f_{g k}^{-}(x) ; d=1,2, \ldots, \frac{D}{2} \quad(3.74 a) \\
& \phi_{g d}(x, y, \hat{\Omega})=\sum_{n=1}^{N} \Delta_{n} \sum_{k=1}^{K} \phi_{g n d k}(y) f_{g k}^{+}(x) ; d=\frac{D}{2}+1, \ldots, D \quad(3.74 b)
\end{aligned}
$$

The approximate multigroup scalar flux, which is of primary interest in reactor problems, is then determined from

$\phi_{g}(x, y)=\sum_{n=1}^{N} \Delta_{n} \sum_{k=1}^{K}\left[\sum_{d=1}^{D / 2} \phi_{g n d k} f_{g k}^{-}(x) w_{d}+\sum_{d=\frac{D}{2}+1}^{D} \phi_{g n d k}(y) f_{g k}^{+}(x) w_{d}\right]$

F'or approximate three-dimensional solutions, the steps are analogous, with the base functions being generated from related twodimensional problems, and the weighted synthesis constants being calculated from the formulas in Appendix B. 
CHAPTER IV

COMPUTER CODES USED

In order to demonstrate that the variational synthesis method developed in Chapter III is a valid and useful technique for obtaining approximate solutions to the transport equation, an experimental computer code has been written to obtain the solutions for the twodimensional case. The two-dimensional case has been chosen to test the method primarily because direct two-dimensional solutions may be obtained to provide comparisons by which to check the accuracy of the synthesized solution. The similarities between the reduced equations for the two-dimensional case and the three-dimensional case are sufficient to suggest that if the two-dimensional application is successful, the three-dimensional application should likewise be successful.

In that the method, as developed, seeks to remove a spatial variable by a synthesis trial function chosen for each energy grous, the adequacy of the spatial nature of the approximation has been investigated by considering only a single energy group. The primary question arising in the extension from one to many energy groups is in regard to the coupling between groups through the scattering source term, which requires that the scattering cross section be weighted over the forward and adjoint base functions from different energy groups. The same type cross-weighting, over forward and adjoint base functions from the two different half-ranges of $\Omega_{x}$, must be utilized in the scattering source term for the one-group problem, however, and a demonstration of its 
applicability will be sufficient to infer the applicability of the groupwise cross-weighting necessary in the mult1group problem.

\section{The TS2 Experimental Computer Code}

The experimental computer code developed for the demonstration of the applicablilty of the transport synthesis method is the TS2 code, which is written in IBM Fortran IV, H level for the IBM 360 models 75 and 91 and requires, for the type of sample problems investigated in the work, storage capacity of $256 \mathrm{~K}$ four-byte words. The large numizer of matrix manipulations required in the solution for the mixing function has necessitated the use of double-precision arithmetic which allows a numertcal accuracy of approximately fourteen significant figures. The normal six digit accuracy of single-precision arithmetic in the IBM 360 series can be the cause of significant machine round-off errors, particularly in the operation of matrix inversion. Auxiliary equipment required by the TS2 code consists only of a card reader, printer, and one disk used for temporary storage in passing input information between the first and subsequent cases of a single run.

The TS2 code accepts, as punched card input, the normal geometric and cross-sectional description of the reactor to be calculated, p].us the base functions to be used in the synthesis approximations. The code utilizes the input information to calculate the matrix synthesis constants and the finite difference form of the reduced matrix transport operator. After inversion and storage of the reduced matrix transport operator, the code solves for the discrete angular mixing functions, by the iteration procedure described in Chapter III, and combines these with the base functions to obtain the approximate scalar flux by use of 
Equation 3.75. Only inner iterations, as described in Chapter III, are required, since the code considers a single energy group. Using the approximate scalar fluxes, the code then calculates neutron reaction rates for each material in the reactor in a form comparable to those obtained from codes used to determine the direct solution. The approximate angular fluxes as given by Equations $3.74 \mathrm{a}$ and $3.74 \mathrm{~b}$ may be obtained by input option.

The equations for the synthesis approximation have been developed assuming the existence of an inhomogeneous source term, $\mathrm{S}(\overrightarrow{\mathrm{r}}, \hat{\Omega}, \mathrm{E})$, in the transport equation. If the source term in the transport equation is homogeneous, that is, dependent on the flux $\Psi$ as in the presence of fissioning material, then a non-trivial solution can be found only if there exists an adjustable parameter, or eigenvalue, for the problem. Since in many reactor applications, the source of neutrons is due to fission reactions, the TS2 code has been programed to solve the eigenvalue problem for the special case where the ratio of scattering to fission cross section is constant over the reactor being considered. In this special case, the combined scattering and fission source term in the monoenergetic transport equation may be written as

$$
\text { Source }=\frac{1}{c} \int_{4 \pi} \int_{\pi} d \hat{\Omega}\left[\Sigma_{s}(\vec{r})+\nu \Sigma_{f}(\vec{r})\right] \Psi(\vec{r}, \hat{\Omega})
$$

where $\nu$ is the number of neutrons produced per fission, $1 / c$ is the eigenvalue and the scattering is assumed isotropic. The reduced synthesis equations to be solved are then identical to Equations $3.46 \mathrm{a}$ and $3.46 \mathrm{~b}$, with the source at mesh increment, I, now given by 
$\frac{1}{c} S_{n I}^{\alpha}=\frac{1}{c}\left\{\sum_{d^{\prime}=1}^{D / 2}\left(\Sigma_{s}+\nu \Sigma_{f}\right)_{n m}^{\alpha-} w_{d^{\prime}} \phi_{n d^{\prime} I}+\sum_{d^{\prime}=I / 2+1}^{D}\left(\Sigma_{s}+\nu \Sigma_{f}\right)_{n m}^{\alpha+} w_{d^{\prime}} \phi_{n d^{\prime} I}\right\} ;$

$$
\alpha=+,-(4.2)
$$

and the matrix constants now having elements given by

$$
\begin{array}{r}
\left(\Sigma_{\mathrm{s}}+\nu \Sigma_{\mathrm{f}}\right)_{\mathrm{nm}}^{\alpha \beta j \mathrm{k}}=\int_{\mathrm{x}_{\mathrm{n}}}^{\mathrm{x} \mathrm{n}_{\mathrm{l}}} \mathrm{f}_{\mathrm{j}(\mathrm{m})}^{\alpha}(\mathrm{x})\left[\Sigma_{\mathrm{sm}}(\mathrm{x})+\nu \Sigma_{\mathrm{fm}}(\mathrm{x})\right] \mathrm{f}_{\mathrm{k}(\mathrm{m})}^{\beta}(\mathrm{x}) \mathrm{dx} \\
\alpha, \beta=+,-\quad(4.3)
\end{array}
$$

The iteration procedure used is the same as for the fixed source calculation, beginning with a first guess for the solution and the eigenvalue, and refining the eigenvalue estimation at the end of each iteration, $t$, by the relation

$$
\left(\frac{1}{c}\right)^{t}=\left(\frac{1}{c}\right)^{t-1} \frac{\sum_{n=1}^{\mathbb{N}} \sum_{I=1}^{\text {IMAX }}\left(s_{n I}^{+}+s_{n I}^{-}\right)^{t-I}}{\sum_{n=1}^{N} \sum_{I=1}^{\text {TMAX }}\left(s_{n I}^{+}+s_{n I}^{-}\right)^{t}}
$$

Having solved by iteration for the discrete angular mixing functions and the eigenvalue, $1 / c$, the approximate scalar flux, $\phi(x, y)$, is then calculated and used by the TS2 code to determine the usual eigenvelue, the effective multiplication factor $\mathrm{k}_{\mathrm{eff}}$, through the relation 


$$
\frac{1}{k_{\text {eff }}}=\frac{1}{c}+\left(\frac{1}{c}-1\right) \frac{\int_{0}^{x_{N+1}} d x \int_{0}^{y_{M+1}} d y \Sigma_{s}(x, y) \phi(x, y)}{\int_{0}^{x_{N+1}} d x \int_{0}^{y_{M+1}} d y \Sigma_{f}(x, y) \phi(x, y)}
$$

Consideration of the eigenvalue problem only for the special case with constant ratio of scattering to fission cross section was prompted by the usefulness of the fixed source iteration procedure in that case, and does not imply a limitation on the applicability of the varfational synthesis method. Treatment of the general eigenvalue: problem for neutron production through fission reactions would require a more complicated iteration procedure, necessitating considerable programing modifications to the TS2 code.

\section{Computer Codes Used for Obtaining Direct Solutions}

Several computer codes, other than the experimental TS2 synthesis code, have been used in this investigation. These codes, to be referred to by name in subsequent discussion, were used to obtain direct solutions to the problems considered for comparison purposes and to oktain base functions for use in the synthesis approximation. Their names, descriptions, and use in this investigation are as given below.

ANI $\mathrm{SN}^{46}$-- A transport-theory code which solves the one-dimensional Boltzmann equation in the direct multigroup discrete-ordinates approximation. Its use was to obtain solutions to related one-dimensional problems and produce angular fluxes to be used as the base functions, $f_{k}^{ \pm}(x)$, in the synthesis approximation. Since, for the oneenergy-group problem, the forward and adjoint solutions of the Boltzmann 
equation are related by

$$
\Psi(\mathrm{x}, \hat{\Omega})=\Psi^{*}(\mathrm{x},-\hat{\Omega})
$$

a single ANISN calculation produces both the forward and adjoint base functions from an axial zone. For the purpose of this investigation, ANISN was modified slightly to output the angular fluxes in punched card form acceptable to the TS2 synthesis code.

$D_{0} T^{47}$-- A transport-theory code which solves the two-dimensional Boltzmann equation in the direct, multigroup discrete ordinates approximation. Its use was to obtain direct solutions to the problems considered and allow a determination of the accuracy of the approximate synthesis solution by comparison. Observation of the computer tirnes required to obtain the direct and synthesized solutions also gave a comparative value of the cost of the synthesis calculation and an indication of the feasibility of using the synthesis method as a means of reducing the cost of routine design calculations. All computing times observed are directly comparable to the synthesis computing time for fixed source calculations since the same iteration procedure is used. In solving the general elgenvalue problem, however, DOT normally uses an inner and outer iteration procedure (ever for the one-group case). For the purposes here, all DOT eigenvalue calculations were obtained first by the normal iteration procedure to determine the solution and the eigenvalue, $\mathrm{k}_{\text {eff' }}$, for comparative purposes and then redone, considering scattering and fissions as a single source and calculating the eigenvalue $1 / \mathrm{c}$. In the second of these calculations, the inner-outer iteration procedure is not required and the resultant computing time 
is directly comparable to the synthesis computing time. Both iteration procedures result, of course, in the same solution, except for a normalization factor which is automatically determined. EXTERMINATOR ${ }^{48} \rightarrow$ A code which solves the two-dimensional Boltzmann equations in the multigroup, diffusion-theory approximation. In that the diffusion-theory approximation is the most widely used method for obtaining approximate neutron distributions, diffusion solutions to the problems considered have been obtained for comparison with the direct and synthesized transport solutions. EXTERMINATOR is em order code which, by present standards, is slow and inefficient and its computing times are longer than to be expected from more modern diffusion codes. It has been used to obtain comparative solutions in the diffusion approximation for the fixed source problems considered. CITATION ${ }^{48}$-- A code which solves the multidimensional Boltzmann equation in the multigroup, diffusion-theory approximation. It is a modern and efficlent code which has been used to obtain comparative diffusion-theory solutions for the eigenvalue problems considered. Computing times required for the CITATION solutions provide a valid comparison between the cost of the direct transport solution from the code DOT, the cost of the synthesis solution and the cost of the most efficient diffusion-theory solution. No provision is currently available in CITATION for obtaining diffusion-theory solutions to fixed source problems.

Each of these computer codes was modified, for the purpose of this investigation, so that the computing time in seconds was output for each calculation. 


\section{Guidelines Used in Comparisons}

In the determination of the accuracy of the approximate synthesized solution by comparison to the direct transport and diffusion solutions, care has been taken to assure that the spatial finite difference mesh used to obtain each solution was consistent with that used for all others. This requires, in the synthesis calculation, that the mesh chosen along the $y$-axis for the determination of the mixing f'unctions be identical to mesh along the $\mathrm{y}$-axis in the direct transport and diffusion calculations. It also requires that the mesh choser. along the $x$-axis for the calculation of the base functions with the code ANISN be identical to the mesh along the $\mathrm{x}$-axis of the direct transport and diffusion calculations.

The discrete angular segmentation utilized in the synthesis solutions and the direct discrete ordinates solutions has been made consistent by employing the same discrete weights and cosines, $\mathrm{w}_{d}, \mathrm{H}_{\mathrm{d}}$ and $\eta_{d}$, in the comparison cases for like problems. This segmentation has been chosen to be symetric in all calculations by requiring the weights, $w_{d}$, of all segments to be equal. The standard notation for the angular segmentation, $S_{n}$, will therefore be used to describe the order of the angular segmentation in the synthesis calculations as well as the direct discrete ordinates calculation. In this notation the subscript $n$ of $S_{n}$ implies that the number of discrete segments per octant of the unit sphere is given by $\frac{n}{8}(n+2)$. For two-dimensional problems, the angular flux solution must be symetric about the plane being considered, hence only four octants need be considered and the 
subscript $n$ implies that the total number of discrete angular seguents required is

$$
D=\frac{n}{2}(n+2)
$$

In referring to the angular segmentation used in the code ANISN for the generation of the base functions, the standard $S_{n}$ notation. implies, by the symmetry of the one-dimensional problem, that there are $\frac{n}{2}$ segments in each of the half ranges of $\Omega_{x}$, and a combined total of $n$ segments.

In subsequent discussion, all the base functions obtained from a single ANISN calculation will be referred to as a set of base functions. Thus, for example, a set of base functions from an $\mathrm{S}_{4}$ ANISN calculation will consist of four functions, the angular flux solutions for the four discrete segments, and will be used in the synthesis calculation as two $f_{k}^{-}(x)$ and two $f_{k}^{+}(x)$.

There is no required correlation between the $S_{n}$ order of the ANISN calculation producing the base functions, and the $S_{n}$ order used in the subsequent synthesis solution for the mixing functions. The natural choice, however, of consistent angular order between the AIIISN and synthesis calculations has been used in this investigation with only one exception. It was felt that this choice will probably produce the most accurate approximate solution when the $\mathrm{n}$ discrete cosines in the ANISN calculation are the same as the discrete $x$-direction cosines in the synthesis calculation.

In the comparison diffusion-theory calculations, surfaces with vacuum boundary conditions are treated by requiring the extrapolated 
scalar flux to vanish at a distance $0.71 \lambda_{\mathrm{tr}}$ from the edge of the surface. This is the normal boundary condition imposed on the solution in the diffusion approximation, and is consistent with the transport boundary condition at a free surface of infinite extent. No modifications to the standard diffusion-theory approximation have been employed in these comparison cases.

A meaningful computing time compariscon between the various calculations for a given problem may be made only if the iterative process in each calculation has reached the same convergence level. The iterative procedures in the DOT, CITATION and EXTERMINATOR calculations were all terminated when the maximum relative pointwise error in the scalar flux had been reduced to $1.0 \times 10^{-4}$. An equivalent convergence level in TS2 calculation of the discrete angular mixing functions is obtained by terminating the iterative procedure when the maximum relative error in the elements of the matrix quantity

$$
\sum_{d=1}^{D} \phi_{n d-I^{w}}
$$

for any $n$ and $I$, is reduced to $1.0 \times 10^{-4}$. The ANISN calculations for the determination of the base functions were converged to a relative pointwise scalar flux error of $1.0 \times 10^{-5}$ to assure that any inaccuracies in the synthesized solution were not the result of poorly converged base functions.

Since the synthesis approximation requires, in addition to the solution for the mixing functions, the solutions to related one-diraensional problems for obtaining base functions and the calculation of 
the matrix synthesis constants, the total computing time for each synthesis calculation is considered to include the time required to generate the base functions, the time required to calculate the synthesis constants, and the iteration time required to solve for the mixing functions. For the direct transport and diffusion solutions, the computing time is considered to be only the time required to converge the iteration procedure. The time required for input and output operations is considered incidental, and not included in the computing time por any of the calculations. 


\section{CHAPTER V}

\section{DISCUSSION OF RESULTS}

\section{Types of Problems Considered}

The variational synthesis method has been used to calculate approximate solutions to the Boltzmann equation for several test problems characteristic of the type encountered in the reactor field for which the diffusion approximation is often inadequate. These are small assemblies with vacuum boundary conditions representative of fast critical assemblies often used in experimental reactor physics studies, and reflected cell problems representative of periodic lattices in large reactor cores. The consistent variational synthesis treatment of reflected boundary conditions, not considered in the development of Chapter III, is the subject of Appendix C. One further problem, representative of the type of problems encountered in radiation shielding work, was considered in this investigation in connection with the examination of the adequacy of the discrete segmentation treatment of the angular variable.

Several approximate synthesis solutions to each of the problems considered have been obtained with the TS2 code by using different numbers and choices of base functions, different channel segmentation of the base function axis, $x$, and different angular segmentation. These various solutions were obtained in order to observe the sensitivity of the accuracy and computing time of the synthesis method to 
the amount and use of the a priori knowledge on which the approximation is based.

The sensitivity to the choice of adjoint base functions, ors weight functions, is of considerable interest since, for the multigroup problem, the best choice would require that both forward and adjoint ANISN calculations be performed. In large scale problems, the cost of computing the base functions may be a large fraction of the total cost of the synthesis solution and the adjoint ANISN calculations may be avoided by using the Galerkin weighting method. 32 In this method the adjoint base functions are chosen to be the same as the forward base functions. If the accuracy of the solutions from the Galerkin weighting method is close to that of the variational weighting method re:quiring the adjoint ANISN calculation, the cost of an adequate approximate solution may be significantly reduced. In the application of the synthesis method to diffusion-theory calculations, experience has shown that use of the Galerkin method yields results with accuracy very close to that of the variational weighting method.

The accuracy of these various synthesis calculations for each problem has been determined by comparisons to the results of calculations with the direct finite-difference $S_{n}$ code DOT. The comparative accuracy of the diffusion-theory approximation has also been determined by use of the diffusion codes EXTERMINATOR or CITATION.

Test Problem Results

Problem One

The first problem chosen for calculation by the transport synthesis method is the small ten centimeter square assembly with vacuum 
boundary conditions and macroscopic constants as shown in Figure 2. Diffusion theory is known to be inaccurate for small systems of this type, however this problem is simple enough to allow some basic characteristics of the symthesis method to be observed. For example, the true scalar flux is symmetric with respect to a 90-degree rotation, but the nature of the synthesis trial function expansion tends to destroy the symmetry. Consequently, an observation of the asymmetry in the synthesized scalar $f l u x$, such as the ratio of the top-center to left-center edge flux, is an indication of the error inherent to the method itself. Also, this problem has been extensively used to detect programing errors in the TS2 code, since the first iteration of the fixed source symthesis calculation can be obtained analytically if analytic expressions are available for the base functions.

The first series of cases run for this problem were fixed source calculations utilizing a single set of base functions generated from an $\mathrm{S}_{4}$ ANISN fixed source calculation. The angular quadrature consisted of 12 equal segments, corresponding to an $S_{4}$ quadrature in standard discrete-ordinates terminology. Cases were run using a single channel with variational and Galerkin weighting to observe the effects of different weighting functions.

Results of these runs are given in Table 1 and Figure 3 . It will be observed that both the variational and Galerkin weighting methods appear to give accurate results for integral quantities. The results using variational weighting are seen to be slightly superior in both the integral quantities and the symmetry of the scalar flux. The pointwise scalar fluxes from both methods are very near those of 


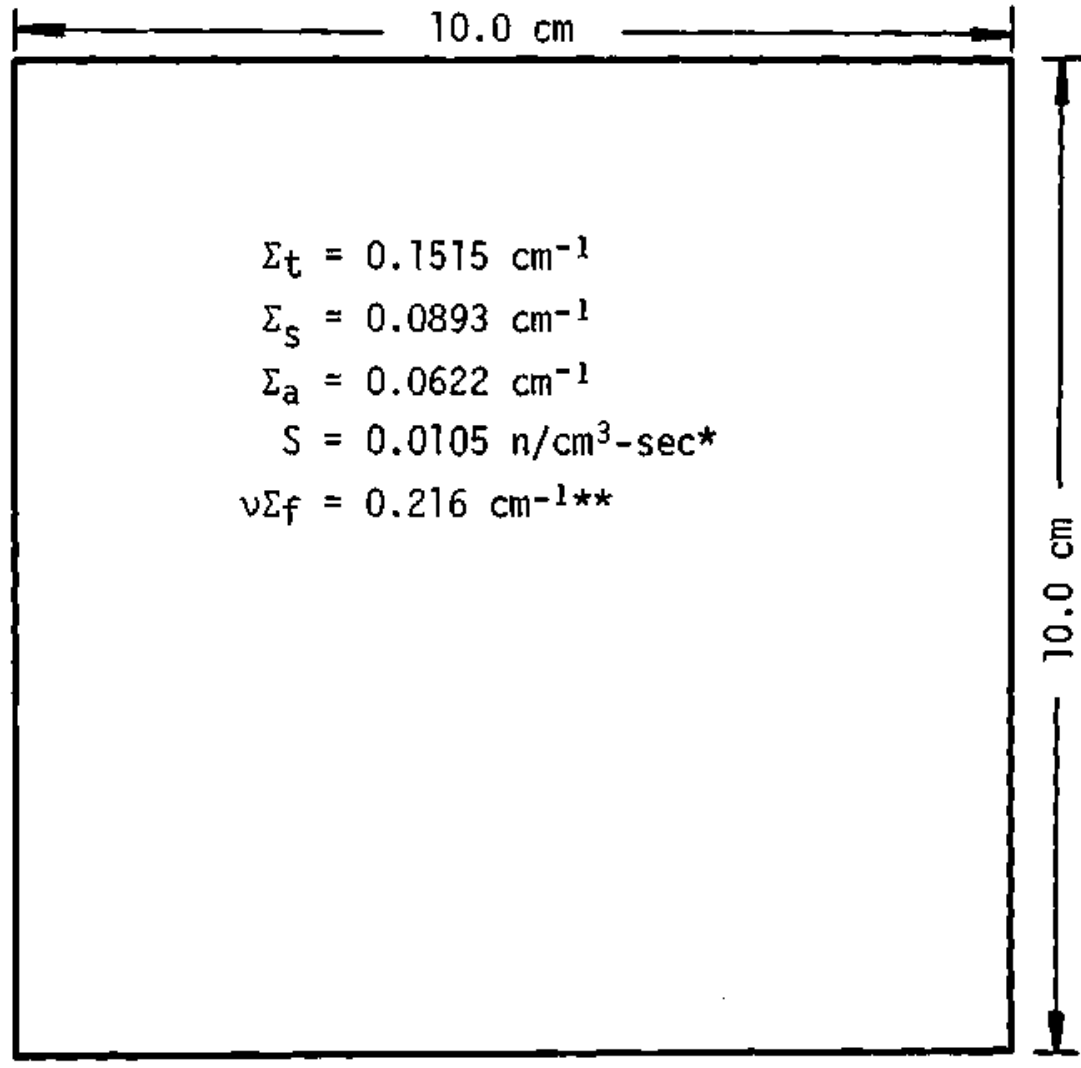

\footnotetext{
*
}

* FIXED SOURCE CALCULATIONS ONLY

EIGENVALUE CALCULATIONS ONLY

Figure 2. Geometry and Macroscopic Constants for Problem One 
Table 1. Results for Fixed Source Problem one with $S_{4}$ and $S_{6}$ Angular Quadrature

\begin{tabular}{|c|c|c|c|c|c|c|c|c|}
\hline & \multicolumn{4}{|c|}{$\mathrm{S}_{4}$ Angular Quadrature } & \multicolumn{4}{|c|}{$S_{6}$ Angular Quadrature } \\
\hline & \multirow[b]{2}{*}{$\begin{array}{c}\text { DOT } \\
\text { Results }\end{array}$} & \multicolumn{3}{|c|}{ Percentage Deviation from DOT Results } & \multirow[b]{2}{*}{$\begin{array}{c}\text { Dor } \\
\text { Results }\end{array}$} & \multicolumn{3}{|c|}{ Percentage Deviation from DOT Results } \\
\hline & & $\begin{array}{l}\text { Synthesis, } \\
\text { Variational } \\
\text { Weighting }\end{array}$ & $\begin{array}{l}\text { Synthesis, } \\
\text { Galerkin } \\
\text { Weighting }\end{array}$ & $\begin{array}{l}\text { Diffusion } \\
\text { Theory }\end{array}$ & & $\begin{array}{l}\text { Synthesis, } \\
\text { Variational } \\
\text { Weighting }\end{array}$ & $\begin{array}{l}\text { Synthesis, } \\
\text { Galerkin } \\
\text { Weighting }\end{array}$ & $\begin{array}{l}\text { Diffusion } \\
\text { Theory }\end{array}$ \\
\hline Absorption Rate & 0.3425 & $+0.03 \%$ & $-0.40 \%$ & $-6.55 \%$ & 0.3431 & $-0.09 \%$ & $+0.10 \%$ & $-6.73 \%$ \\
\hline Leakage Rate & 0.7075 & $-0.01 \%$ & $+0.20 \%$ & $+3.08 \%$ & 0.7069 & $+0.09 \%$ & $-0.20 \%$ & $+3.27 \%$ \\
\hline Sywmetry Ratio* & 1.00 & $+0.10 \%$ & $+1.70 \%$ & $0.00 \%$ & $1 . \infty$ & $+0.36 \%$ & $+0.37 \%$ & $0.00 \%$ \\
\hline $\begin{array}{l}\text { Computing Time } \\
\text { (seconds) }\end{array}$ & 9.22 & 2.02 & 1.84 & 14.58 & 16.99 & 3.42 & 3.44 & 14.58 \\
\hline
\end{tabular}

* Ratio of left edge center to top center scaler flux. 


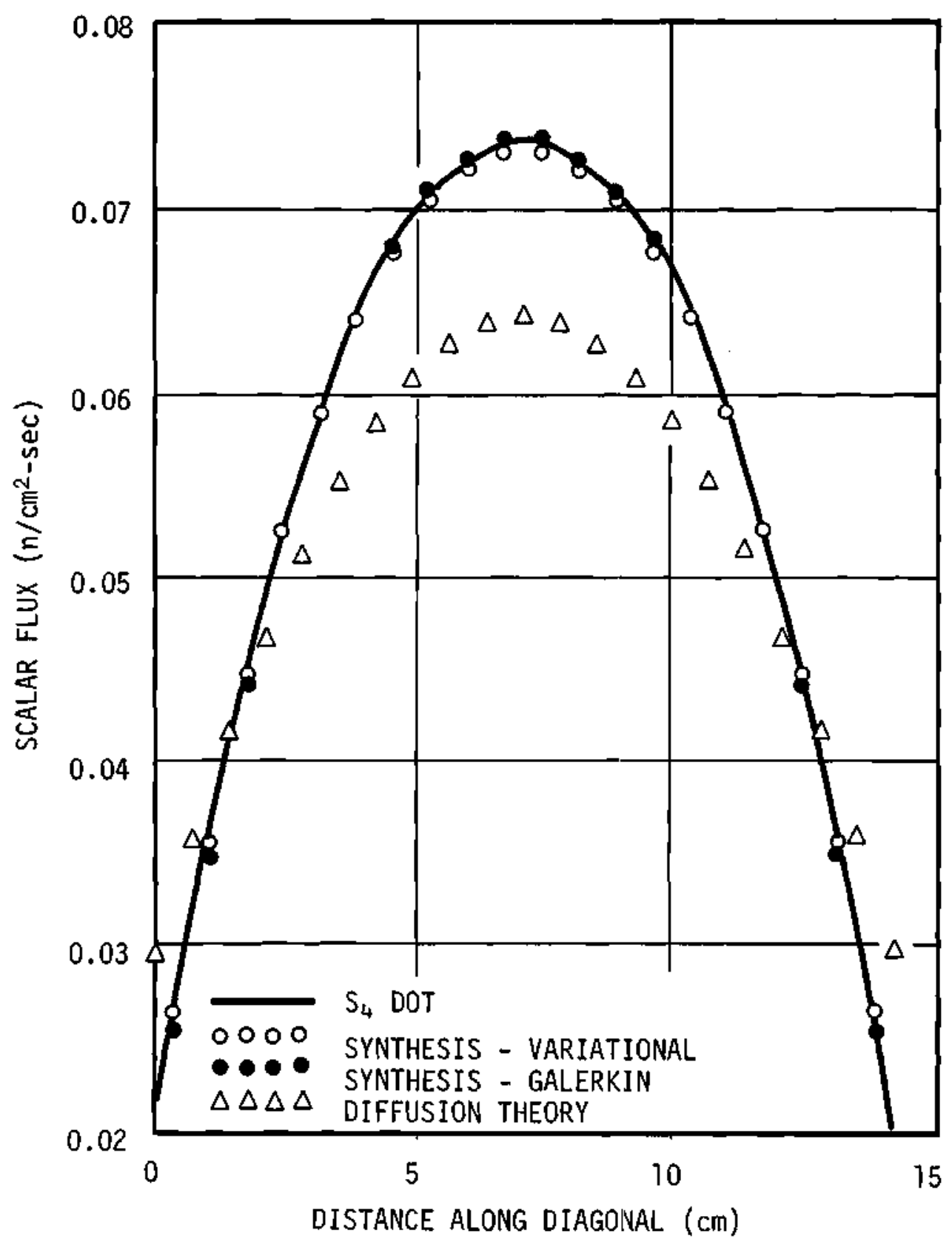

Figure 3. Scalar Flux Along Diagonal for Fixed Source Problem One 
the direct fintte-difference results, and are superior to those calculated from diffusion theory. For this case the synthesis method enjoys a computing time advantage over the direct $S_{n}$ method, but use of the code EXT'ERMINATOR precludes a meaningful computing time comparison for the diffusion-theory calculation.

To demonstrate that the method is also applicable to higherorder angular approximations, the same cases were run using 24 equal angular segments, corresponding to an $\mathrm{S}_{6}$ angular approximation in standard notation. These runs utilized a single channel with one set of $S_{6}$ base functions and the same two weighting methods. Integral. results, also shown in Table 1 , indicate that the synthesis method is also useful for higher-order angular quadrature.

To demonstrate the ability of the multichannel synthesis method to produce accurate results when a poor cholce of base functions is made, another series of fixed source $S_{4}$ calculations for this problem has been performed using base functions of the following analytic form:

$$
\begin{gathered}
f_{1}^{+}(x)=1-e^{-\Sigma_{t} x / 0.7071} \\
f_{1}^{-}(x)=1-e^{-\Sigma_{t}(10-x) / 0.7071}
\end{gathered}
$$

where the value of $\Sigma_{t}$ is as given in Figure 2. For these cases the base functions were hand calculated but could have been obtained, for example, from an $S_{2}$ ANISN calculation for a ten centimeter purely $a b-$ sorbing slab with discrete directions at 45 and 135 degrees from the positive $x$-axis. The channel structures utilized for this series of calculations consisted of five, four, three, two, and one segment of 
the $x$-axis. For each of the runs the channel widths were equal except for the three channel case where the first and third channels were three centimeters wide and the second channel was four centimeters wide. Integral results for this series of calculations are shown in Table 2. It will be observed that while the single channel results are relatively poor, the results approach the direct finite difference $S_{4}$ results with increasing numbers of channels. It is also observed that the computational cost of the synthesis calculation increases with increasing channels and at some point the cost of synthesis calculation will approach that of the direct finite-difference calculation. Ihis clearly indicates that the most accurate and least expensive synthesis solutions will be obtained by using base functions chosen as carefully as possible.

Figure 4 shows a plot of the percentage deviations from the DOT results in the one and five channel synthesis and the diffusion-theory scalar fluxes along the diagonal. For the five channel symthesis, scalar fluxes are in error by less than one per cent except at the corners. Even for the one channel case the results are seen to be supertor to the diffusion-theory results.

Calculations of the eigenvalue for Problem one were performed in two series of $S_{4}$ synthesis calculations. The first series utilized a single set of base functions from an $S_{4}$ ANLSN eigenvalue calculation. Runs were made using one, two, three, and four channels with variational weighting and the same channel widths as were used for the fixed-source calculations of this problem. For the second eigenvalue series, the same calculations were made using base functions from the 
Table 2. Results for Fixed Source Problem One with Analytic Base Functions

\begin{tabular}{|c|c|c|c|c|c|c|c|}
\hline & \multirow{3}{*}{$\begin{array}{c}\text { DOT } \\
\text { Results }\end{array}$} & \multicolumn{6}{|c|}{ Percentage Deviation from DOT Results } \\
\hline & & \multicolumn{5}{|c|}{$\mathrm{S}_{4}$ Synthesis, Variational Weighting, N Channels } & \multirow{2}{*}{$\begin{array}{l}\text { Diffusion } \\
\text { Theory }\end{array}$} \\
\hline & & $N=1$ & $N=2$ & $\mathrm{~N}=3$ & $N=4$ & $\mathrm{~N}=5$ & \\
\hline Absorption Rate & 0.3425 & $+5.15 \%$ & $+2.65 \%$ & $+1.89 \%$ & $+1.31 \%$ & $+1.05 \%$ & $-6.55 \%$ \\
\hline Leakage Rate & 0.7075 & $2.55 \%$ & $-1.30 \%$ & $-0.92 \%$ & $-0.64 \%$ & $-0.51 \%$ & $+3.08 \%$ \\
\hline Symmetry Ratio* & 1.00 & $+21.0 \%$ & $+12.0 \%$ & $+11.0 \%$ & $+8.0 \%$ & $+6.0 \%$ & $0.00 \%$ \\
\hline $\begin{array}{l}\text { Computing Time } \\
\text { (seconds) }\end{array}$ & 9.22 & 0.99 & 2.38 & 3.80 & 5.14 & 6.39 & 14.58 \\
\hline
\end{tabular}

\footnotetext{
* Ratio of left edge center to top center scalar flux.
} 


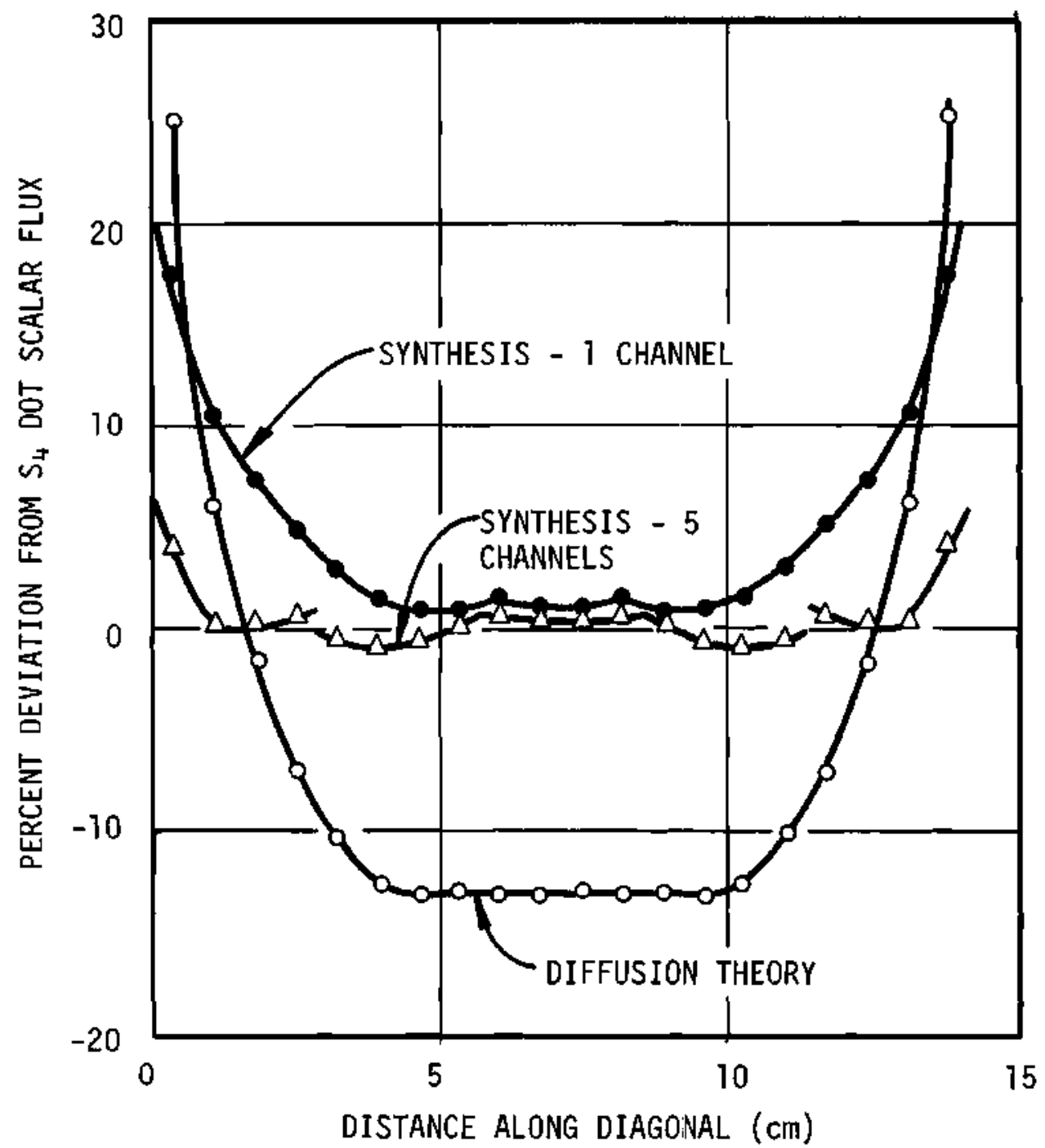

Figure 4. Percentage Deviations in Scalar Flux Along Diagonal 
fixed-source $\mathrm{S}_{4}$ ANISN calculations obtained previously. These base functions are, of course, expected to be relatively poor in comparison to those of the first series.

The results of these two eigenvalue series are shown in Table 3 as percentage deviations from the $S_{4}$ DOT results. It is observed from the first series results that more than a single channel is not necessary to achieve accurate results if the base functions are well chosen. It is also observed that the multichannel synthesis method is captiole of reproducing integral quantities from the direct finite difference DOT calculations to a very high degree. In fact, errors as small as those from the four channel synthesis calculation could be attribuited to differences in convergence levels. For the second series, where a poor choice of base functions was made, it is observed that the results of the single channel synthesis are marginal because of large errors in the peak and mintmum scalar flux values. Using two channels al. Low significant improvement and, as previously observed, increasing the number of channels leads to increased accuracy at the expense of computing time.

The computing times shown here allow a valid comparison betwreen the synthesis calculations and the direct transport and diffusiontheory calculations. It is observed that for the single-channel case, the synthesis calculation is roughly eight times faster than the DOT calculation and slightly faster than the diffusion-theory calculation. Even for the four channel calculation, where the deviations in integral results are practically zero and in pointwise results are less than onehalf per cent, the synthesis calculation is twice as fast as the direct transport calculation. 
Table 3. Results for Eigenvalue Problem One

\begin{tabular}{|c|c|c|c|c|c|c|c|c|c|c|}
\hline & \multirow{4}{*}{$\begin{array}{l}\text { DOT } S_{4} \\
\text { Results }\end{array}$} & \multicolumn{8}{|c|}{ Percentage Devietion from DOT Results } & \multirow{4}{*}{$\begin{array}{l}\text { Diffusion } \\
\text { Theory }\end{array}$} \\
\hline & & \multicolumn{8}{|c|}{ S $_{4}$ Synthesis, Variational Weighting, N Channels } & \\
\hline & & \multicolumn{4}{|c|}{ Series 1} & \multicolumn{4}{|c|}{ Series 2} & \\
\hline & & $\mathrm{N}=1$ & $\mathrm{~N}=2$ & $N=3$ & $N=4$ & $\mathbf{N}=1$ & $\mathbf{N}=2$ & $\mathbf{N}=3$ & $N=4$ & \\
\hline Absorption Rate & 0.3477 & $+0.0584 \phi$ & $-0.0141 \%$ & $+0.0004 g$ & $-0.0006 \%$ & $-0.506 \%$ & $-0.0357 \%$ & $-0.0374 x$ & $-0.0086 \%$ & $-17 \cdot 20$ \\
\hline Leakege Rate & 0.6523 & $-0.0342 \%$ & $+0.0044 \%$ & $-0.0009 \%$ & $-0.0003 \%$ & $+0.265 \%$ & $+0.0159 \%$ & $+0.0168 \%$ & $+0.0015 \%$ & $+9.16 \%$ \\
\hline Eigenvalue, $k_{\text {eff }}$ & 1.2076 & $+0.0613 \%$ & $-0.0108 \%$ & $-0.0008 \%$ & $+0.0003 \%$ & $-0.504 \%$ & $-0.0323 \%$ & $-0.0306 \%$ & $-0.0050 \%$ & $-9.90 \%$ \\
\hline Peak Flwx & 0.08609 & $+0.316 \%$ & $+0.151 \%$ & $+0.029 \%$ & $-0.030 \%$ & $-6.488 \%$ & $+2.880 \%$ & $-1.645 \%$ & $+0.321 x$ & $-30.10 \%$ \\
\hline Mintmun Flux & 0.02155 & $+0.329 \%$ & $-1.113 \%$ & $-0.869 \%$ & $-0.488 \%$ & $+10.682 \%$ & $+2.607 \%$ & $-0.575 \%$ & $+0.252 \%$ & $+25.9 \alpha_{3}$ \\
\hline $\begin{array}{l}\text { Computing Time } \\
\text { (seconds) }\end{array}$ & 20.40 & 2.27 & 5.55 & 7.02 & 9.02 & 2.29 & 4.35 & $7 \cdot 71$ & 11.13 & 3.12 \\
\hline
\end{tabular}


Problem Two

The second problem used to test the transport synthesis method is the small assembly shown in Figure 5. This problem is the same as Problem one with a strong absorber placed in the lower left-hand corner. Diffusion theory is inadequate for this problem for two reasons. First, because the high leakage causes the angular flux to be significantly anisotropic over a large portion of the total area, and second, because the strong absorber causes large flux gradients which diffusion theory cannot calculate correctly. It will be noted that the scalar flux for this problem does not exhibit the same symmetry as for Problem one.

A series of $\mathrm{S}_{4}$ fixed-source symthesis calculations was performed for this problem which utilized two sets of base functions, one set: from a fixed-source $\mathrm{S}_{4}$ ANISN calculation for each of the two axial zones. Both sets were used in the trial function expansion for each zone. These calculations were performed using variational and Galerkin welghting, first with a single channel and then with two channels having channel widths of four centimeters and six centimeters. Integral results for these calculations and the comparison diffusion-theory calculation are given in Table 4 as percentage deviations from the direct finite difference $S_{4}$ results. Scalar flux plots along the diagonal through the absorber are shown in Figure 6 .

It is observed that the synthesis results using a single channel are quite accurate, since the integral quantities and material reaction rates for both weighting methods differ by less than one per cent from the $\mathrm{S}_{4}$ DOT results. By contrast, the diffusion theory results show deviations as high as 28 per cent in the reaction rates for the absorber 


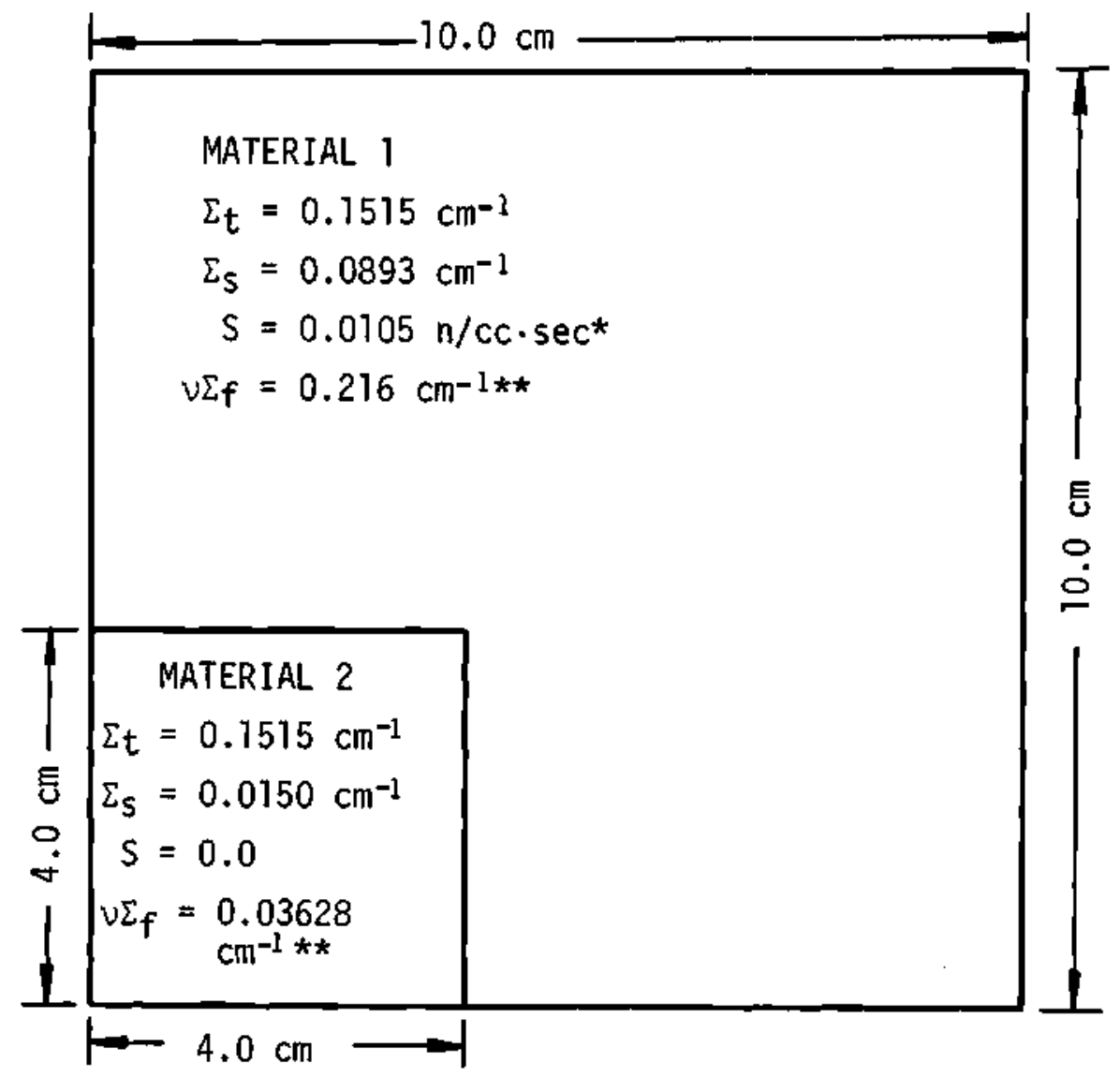

${ }^{*}$ FIXED SOURCE CALCULATIONS ONLY ${ }^{* *}$ EIGENVALUE CALCULATIONS ONLY

Figure 5. Geometry and Macroscopic Constants for Problem Two 
Table 4. Results for Fixed Source Problem Two

\begin{tabular}{|c|c|c|c|c|c|c|}
\hline & \multirow{4}{*}{$\begin{array}{l}\text { DOT } S_{4} \\
\text { Results }\end{array}$} & \multicolumn{5}{|c|}{ Percentage Deviation from DOT Results } \\
\hline & & \multicolumn{4}{|c|}{$\mathrm{S}_{4}$ Synthesis, $\mathrm{N}$ Channels } & \multirow{3}{*}{$\begin{array}{l}\text { Diffusion } \\
\text { Theory }\end{array}$} \\
\hline & & \multicolumn{2}{|c|}{ Variational Weighting } & \multicolumn{2}{|c|}{ Galerkin Weighting } & \\
\hline & & $\mathrm{N}=1$ & $\mathrm{~N}=2$ & $\mathrm{~N}=1$ & $N=2$ & \\
\hline \multicolumn{7}{|l|}{ Totals } \\
\hline $\begin{array}{l}\text { Absorption Rate } \\
\text { Leakage Rate }\end{array}$ & $\begin{array}{l}0.3080 \\
0.5740\end{array}$ & $\begin{array}{l}-0.18 \% \\
+0.10 \%\end{array}$ & $\begin{array}{l}+0.011 \% \\
-0.003 \%\end{array}$ & $\begin{array}{l}-0.03 \% \\
+0.02 \%\end{array}$ & $\begin{array}{l}+0.03 \% \\
-0.014 \%\end{array}$ & $\begin{array}{l}-4.84 \% \\
+2.48 \%\end{array}$ \\
\hline \multicolumn{7}{|l|}{ Material 1} \\
\hline $\begin{array}{l}\text { Absorption Rate } \\
\text { Ieakage Rate }\end{array}$ & $\begin{array}{l}0.2629 \\
0.6191\end{array}$ & $\begin{array}{l}-0.06 \% \\
+0.03 \%\end{array}$ & $\begin{array}{l}+0.014 \% \\
-0.006 \%\end{array}$ & $\begin{array}{l}-0.13 \% \\
+0.05 \%\end{array}$ & $\begin{array}{l}+0.037 \% \\
-0.015 \%\end{array}$ & $\begin{array}{l}-11.58 \% \\
+4.41 \%\end{array}$ \\
\hline \multicolumn{7}{|l|}{ Material 2} \\
\hline $\begin{array}{l}\text { Absorption Rate } \\
\text { Leakage Rate }\end{array}$ & $\begin{array}{r}0.0451 \\
-0.0451\end{array}$ & $\begin{array}{l}-0.90 \% \\
+0.90 \%\end{array}$ & $\begin{array}{l}-0.004 \% \\
+0.004 \%\end{array}$ & $\begin{array}{l}+0.53 \% \\
-0.53 \%\end{array}$ & $\begin{array}{l}+0.006 \% \\
-0.006 \%\end{array}$ & $\begin{array}{l}+28.98 \% \\
-28.98 \%\end{array}$ \\
\hline $\begin{array}{l}\text { Computing Time } \\
\text { (seconds) }\end{array}$ & 3.03 & 0.51 & 1.28 & 0.51 & 1.31 & 4.09 \\
\hline
\end{tabular}




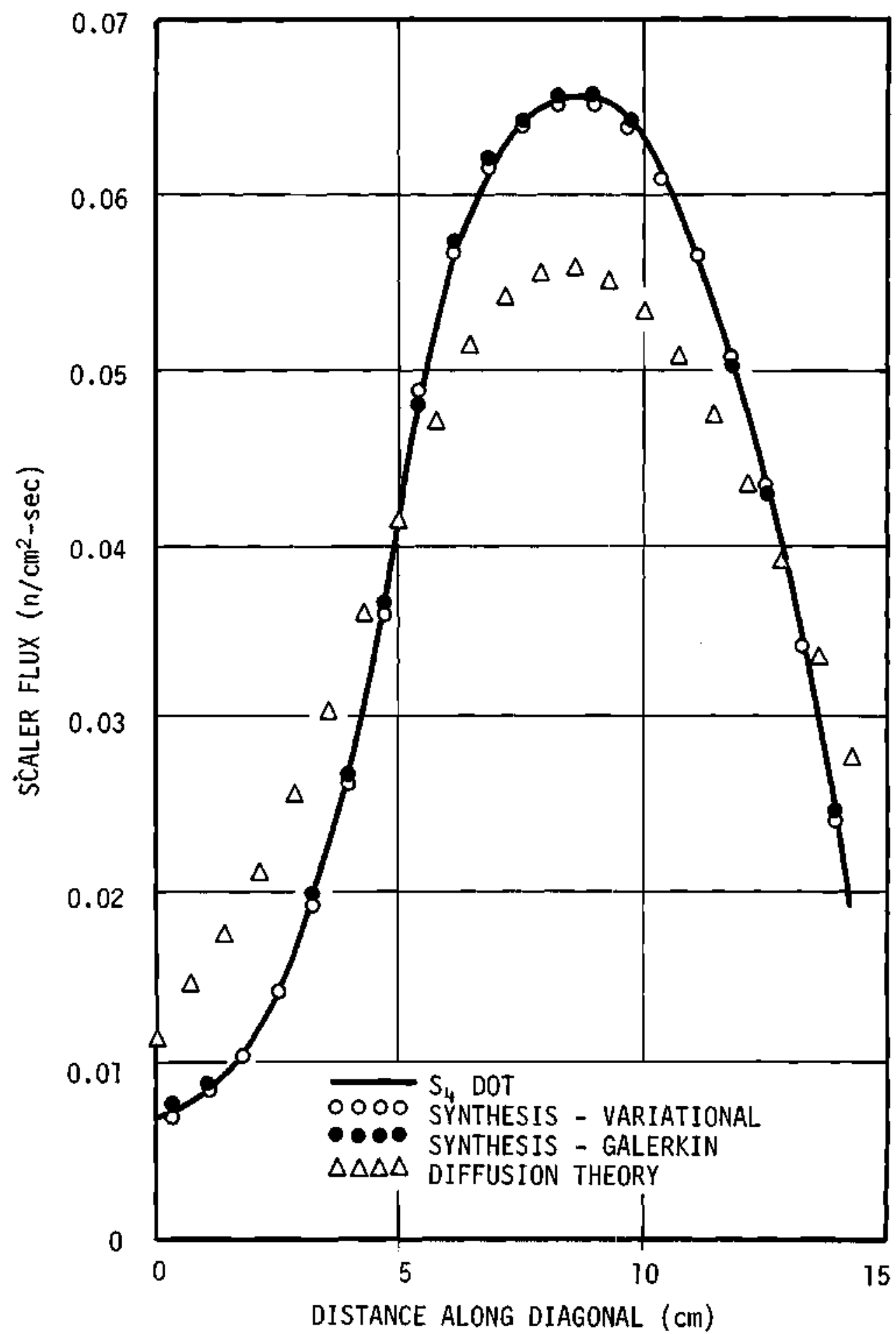

Figure 6. Scalar Flux Along Diagonal for Fixed Source Problem Two 
material. Similar observations can be made about the accuracy of the pointwise scalar fluxes in Figure 6 , which shows that the flux gradients are accurately approximated by the symthesis solution where the diffusion approximation is inadequate. Comparisons of the percentage deviations for the one and two channel synthesis results in Table if shows a significant increase in accuracy by using two channels, particularly for the reaction rates in the absorber material. As observed previously, the comparative accuracies of the variational and Galerkin weighting methods suggest that either method may be used effectively. The computing times given in Table 4 indicate that the sing.le channel synthesis method is roughly six times faster than the direct finite-difference $S_{n}$ method, while the two channel calculation is roughly 2.5 times as fast. Again the use of the code EXTERMINATOR precludes a meaningful computing time comparison between the synthesis method and diffusion theory for fixed source calculations.

Eigenvalue calculations for this problem have also been periormed for the case when the ratio of $\nu \Sigma_{f}$ to $\Sigma_{s}$ is the same in both materials. The first series of eigenvalue calculations was performed using two sets of base functions and 12 angular segments. Base functions were obtained from $\mathrm{S}_{4}$ ANISN eigenvalue calculations for each of the two axial zones. Both sets of base functions were used in each axial zone and variational weighting was used in one, two, three and four channel calculations. The second series was identical with the first except the base functions were used only in their respective axial zones. For the second series, of course, the base functions were switched at the axial zone interface and the resulting scalar flux is discontinuous there. 
Table 5 shows the integral results for these two series of calculations as percentage deviations from the direct finite-difference $S_{4}$ results. Percentage deviations in the diffusion-theory results are also given for comparison. For the first sexies, with no base function switch, the errors in the total integral results for the single channel case are less than one per cent, but in the absorber material the errors are of the order of three per cent. By using two channels, all erurors are reduced below one per cent, and increasing the number of channels to four reduces all errors to less than 0.10 per cent. For the diffusion-theory case, however, errors in the absorber material are very large -- over 40 per cent for the fission source. The scalar flux along the diagonal through the absorber is shown for this series ir. Figure 7. It is observed that the single channel results are far superior to the diffusion results, and that for the four channel case, the pointwise scalar fluxes are very close to those from the direct $\mathrm{S}_{4}$ DOT calculation. Comparison of the computing times indicates that the single channel synthesis is roughly 6.5 times faster than the finitedifference transport calculation and 40 per cent faster than the diffusion-theory calculation. Even when four channels are used the direct calculation requires 40 per cent more computing time than the synthesis calculation

For the second series, where base functions were switched at the axial zone interface, a single channel yields integral results which are in error by roughly ten per cent in the absorber region. Increasing the number of channels reduces these errors, as observed previously, at the expense of running time. In the axial flux plot 
Table 5. Results for Bugenvalue Problen Tro

\begin{tabular}{|c|c|c|c|c|c|c|c|c|c|c|}
\hline & \multirow{4}{*}{$\begin{array}{l}\text { DOT } s_{4} \\
\text { Results }\end{array}$} & \multicolumn{9}{|c|}{ Percentage Deviation from Dor Reaults } \\
\hline & & \multicolumn{8}{|c|}{ 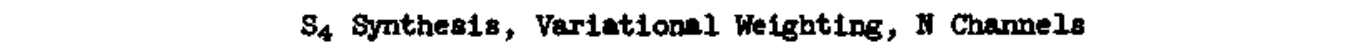 } & \multirow{3}{*}{$\begin{array}{l}\text { Diffusion } \\
\text { Theory }\end{array}$} \\
\hline & & \multicolumn{4}{|c|}{ Serieg 1} & \multicolumn{4}{|c|}{ Serles 2} & \\
\hline & & $\mathrm{N}=1$ & $\mathbf{N}=2$ & $\mathbf{H}=3$ & $\mathbb{N}=4$ & $\mathrm{H}=\mathbf{1}$ & $\mathrm{H}=\mathbf{2}$ & $\mathrm{H}=3$ & $\mathbb{N}=4$ & \\
\hline \multicolumn{11}{|l|}{ Totel } \\
\hline $\begin{array}{l}\text { Absorption Rate } \\
\text { Ieakege Rate } \\
\text { Fission Source }\end{array}$ & $\begin{array}{l}0.37060 \\
0.62940 \\
1.0\end{array}$ & $\begin{array}{l}+0.111 \% \\
-0.065 \% \\
0.0 \%\end{array}$ & $\begin{array}{l}-0.067 \% \\
+0.039 \% \\
0.0 \%\end{array}$ & $\begin{array}{l}+0.040 \% \\
+0.041 \% \\
0.0 \%\end{array}$ & $\begin{array}{l}+0.002 \% \\
-0.001 \% \\
0.0 \%\end{array}$ & $\begin{array}{l}+0.634 \% \\
-0.373 \% \\
0.0 \%\end{array}$ & $\begin{array}{l}+0.050 \% \\
-0.029 \% \\
0.0 \%\end{array}$ & $\begin{array}{l}+0.188 \% \\
-0.11 \% \\
0.0 \%\end{array}$ & $\begin{array}{l}+0.152 \% \\
-0.089 \% \\
0.0 \%\end{array}$ & $\begin{array}{r}-4.55 \% \\
+2.68 \% \\
0.0 \%\end{array}$ \\
\hline \multicolumn{11}{|l|}{ Naterial 1} \\
\hline $\begin{array}{l}\text { Abaorption Rate } \\
\text { Lealage Rate } \\
\text { Flasion Source }\end{array}$ & $\begin{array}{l}0.31449 \\
0.67207 \\
0.98656\end{array}$ & $\begin{array}{l}-0.456 \% \\
+0.135 \% \\
-0.054 \%\end{array}$ & $\begin{array}{l}-0.017 \% \\
+0.010 \% \\
+0.001 \%\end{array}$ & $\begin{array}{l}+0.022 \% \\
-0.003 \% \\
+0.005 \%\end{array}$ & $\begin{array}{l}-0.008 \% \\
-0.002 \% \\
-0.004 \%\end{array}$ & $\begin{array}{l}-1.23 \% \\
+0.326 \% \\
-0.171 \%\end{array}$ & $\begin{array}{l}-0.341 \% \\
+0.103 \% \\
-0.038 \%\end{array}$ & $\begin{array}{l}+0.028 \% \\
-0.039 \% \\
-0.017 \%\end{array}$ & $\begin{array}{l}+0.063 \% \\
-0.045 \% \\
-0.011 \%\end{array}$ & $\begin{array}{l}-10.2 \% \\
+3.94 \% \\
-0.556 \%\end{array}$ \\
\hline \multicolumn{11}{|l|}{ Materiel 2} \\
\hline $\begin{array}{l}\text { Absorption Rate } \\
\text { Leakege Rate } \\
\text { Flssion Source }\end{array}$ & $\begin{array}{r}0.05612 \\
-0.04265 \\
0.01347\end{array}$ & $\begin{array}{l}+3.28 \% \\
-3.15 \% \\
+3.70 \%\end{array}$ & $\begin{array}{l}-0.364 \% \\
+0.370 \% \\
-0.345 \%\end{array}$ & $\begin{array}{l}-0.602 \% \\
+0.598 \% \\
-0.62 \%\end{array}$ & $\begin{array}{l}+0.040 \% \\
-0.043 \% \\
+0.047 \%\end{array}$ & $\begin{array}{r}+11.1 \% \\
-10.7 \% \\
+12.3 \%\end{array}$ & $\begin{array}{r}+2.22 \% \\
-2.12 \% \\
+2.54 \%\end{array}$ & $\begin{array}{l}+1.08 \% \\
+1.09 \% \\
+1.03 \%\end{array}$ & $\begin{array}{l}+0.645 \% \\
-0.667 \% \\
+0.567 \%\end{array}$ & $\begin{array}{r}+26.9 \% \\
+22.6 \% \\
+40.5 \%\end{array}$ \\
\hline Bigenvalue, keft & 1.10699 & $-0.404 \%$ & $-0.019 \%$ & $+0.017 \%$ & $-0.004 \%$ & $-1.06 \%$ & $-0.304 \%$ & +0.0458 & $+0.007 \%$ & $-12.85 \%$ \\
\hline $\begin{array}{l}\text { Conputing Time } \\
\text { (seconds) }\end{array}$ & 18.03 & 2.70 & 5.94 & 9.33 & 12.84 & 3.03 & 6.94 & 10.82 & 14.72 & 3.84 \\
\hline
\end{tabular}




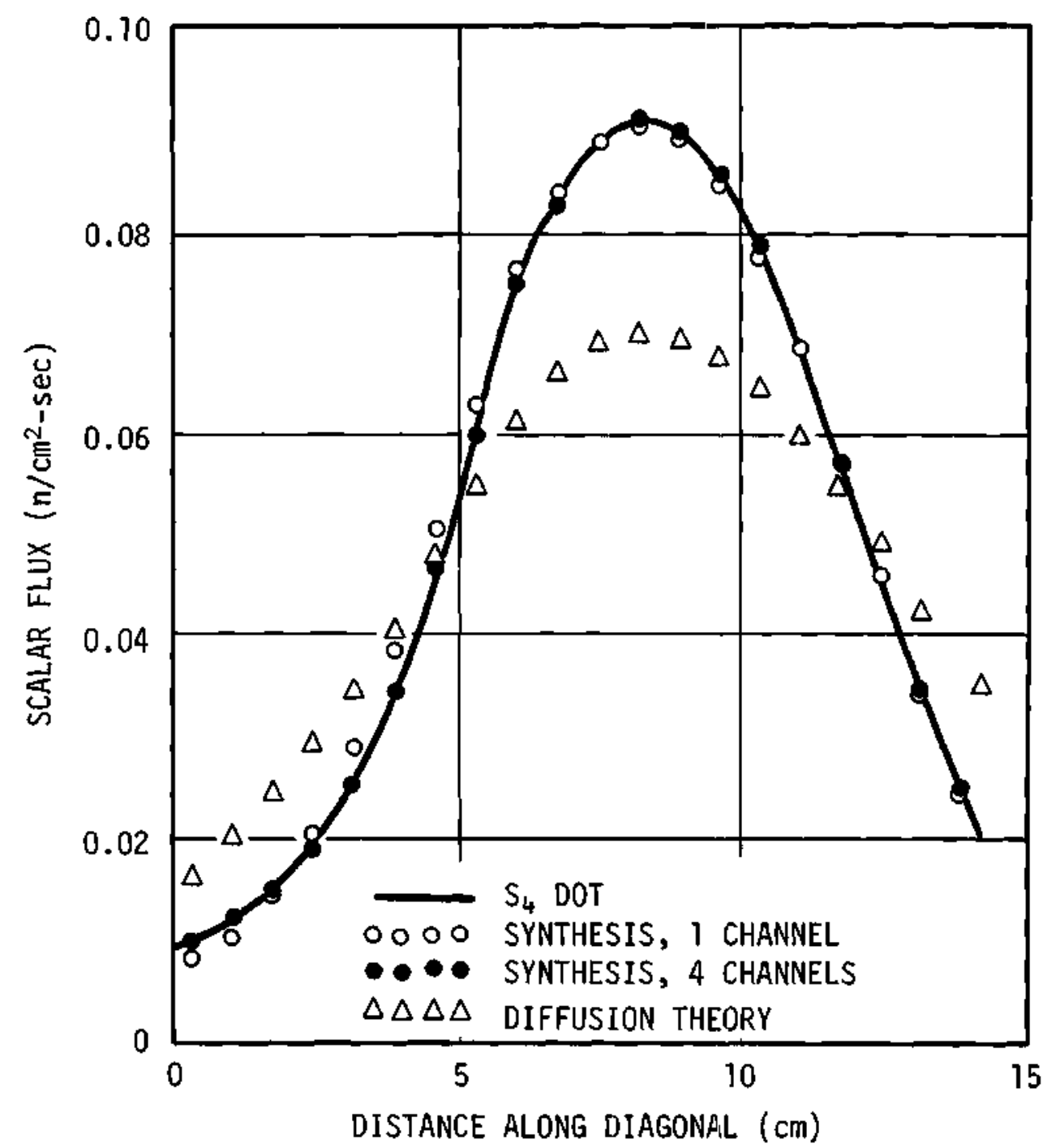

Figure 7. Scalar Flax Along Diagonal for Eigenvalue Problem Two 
through the center of the absorber region, as shown in Figure 8 , the discontinuity at the zone interface is seen to be quite pronounced in the single-channel case, but for the four channel case the discontinulty is barely discernible. This serves to demonstrate again the ability of the multichannel procedure to give pointwise fluxes superior to the single-channel results.

Problem Three

The third problem chosen for calculation by the transport synthesis method is a fixed source problem extracted from the thermal group of a four-group cell problem reported by Wachspress. 49 Although this problem can be calculated adequately by diffusion theory, it is used here because the geometry involved (Figure 9) makes it particlilarly difficult for synthesis approximations removing a spatial variable to produce accurate results.

For simple geometries like that of Problem one, the diffusiontheory solution is separable, and in attempting to synthesize a diffusion-theory solution, a trial function expansion with only one base function can give the exact results. For a geometry as in Figure 9, the diffusion solution is no longer separable, and an accurate diffusion-theory synthesis approximation is more difficult to obtain. This problem is particularly difficult since the asymptotic horizontal fiux shapes for the two axial zones are quite different. Wachspress has thus chosen this problem to demonstrate that his diffusion-theory synthesis method is capable of producing good results when the base functions from two adjacent axial zones are very different. 


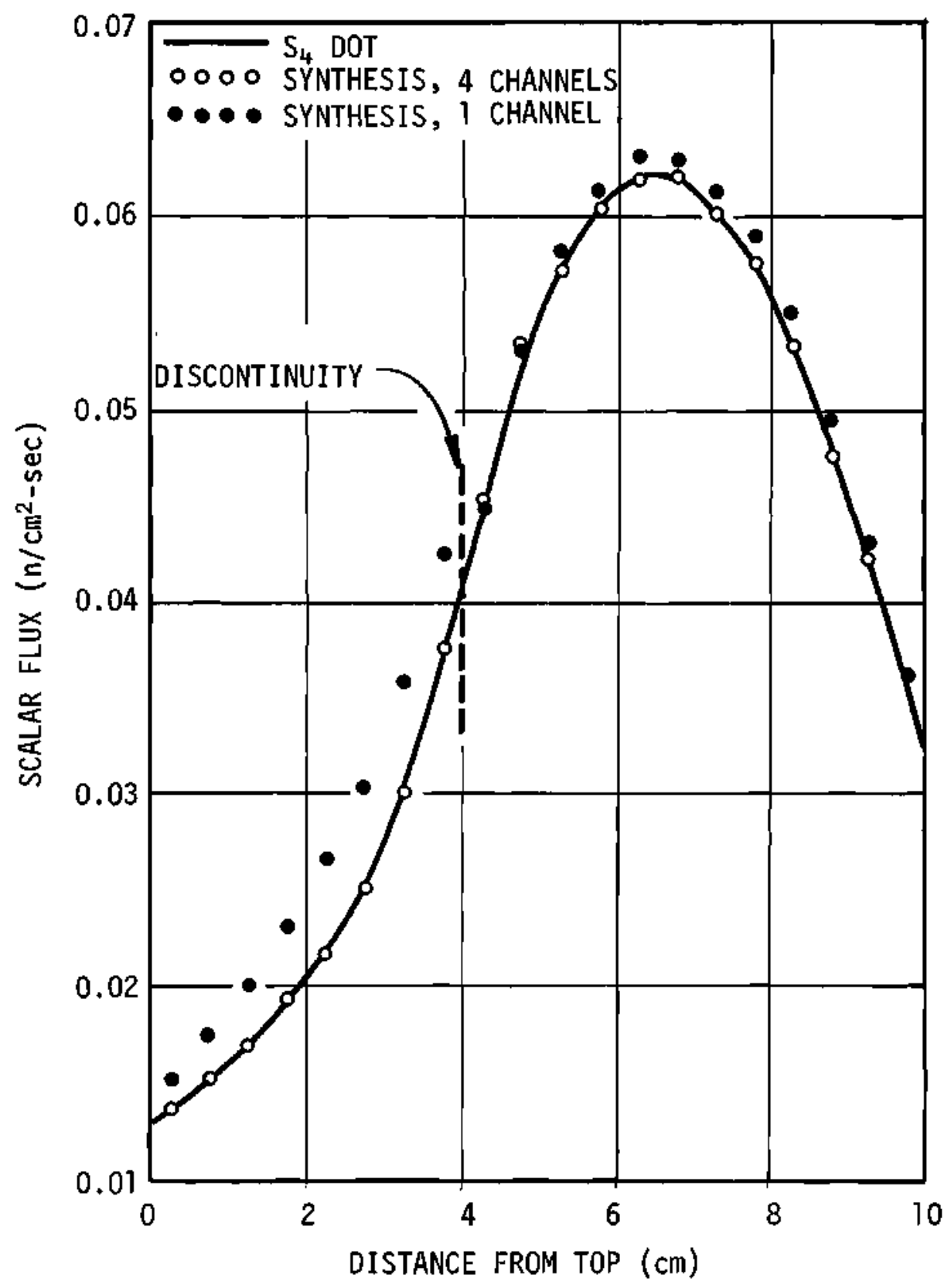

Figure 8. Axial Scalar Flux Through the Center of the Absorber Region 


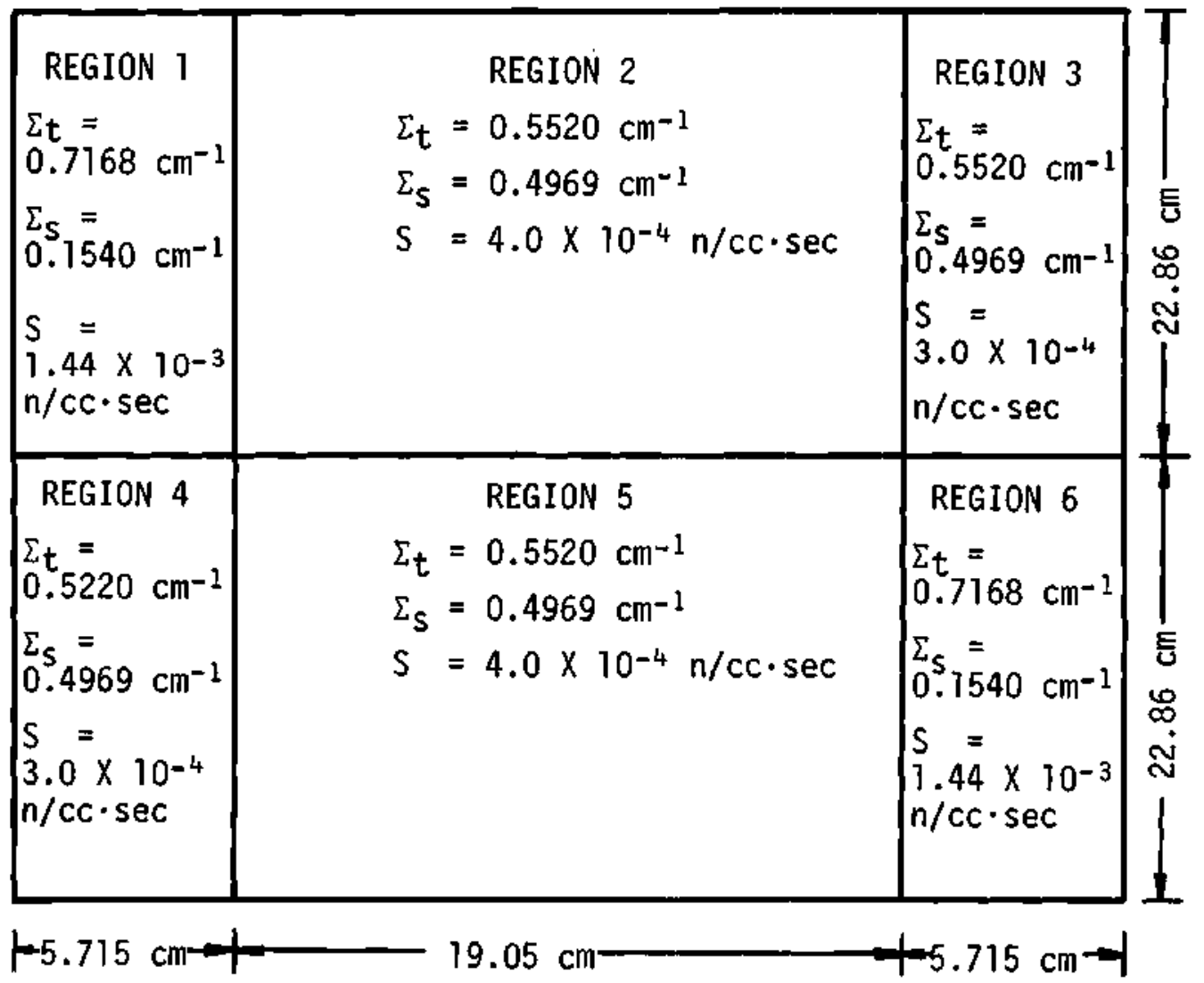

Figure 9. Geometry and Macroscopic Constants for Problem Three 
While the transport-theory solution is not separable even in the simple geometry of Problem one, similarities in the spatial synthesis trial functions make this problem a difficult one for the transport synthesis method also. Three series of $S_{4}$ fixed-source synthesis calculations have therefore been performed to observe the accuracy of the transport symthesis method under conditions where base functions from two adjacent axial zones are very different. For these calculations, three sets of base functions were obtained from $\mathrm{S}_{4}$ ANISN calculations; one set from each of the two axial zones and a third set from an axial homogenization of the two zones.

The first series of calculations used the first two sets of base functions in both axial zones. Runs were made using one, two, three and four channels with channel widths as given in Table 6 . The second series was identical to the first except that each set of base functions was used only in its respective axial zone. For the third series, all three sets of base functions were used in a calculation with only one channel. Here the first two sets of base functions were used in their respective axial zones, along with the third set which was used in both zones. In all three series, the Galerkin weighting method was employed. Integral absorption rates and computing times for these three series of calculations are shown in Table 7. For the first series, deviations in the absorptions by region are seen to be as large as 1.5 per cent for the single channel calculation. Increasing the number of channels improves the accuracy at the expense of increasing the computing time. For the second series, where base functions were useci only in their respective zones, a single channel appears inadequate since 
Table 6. Channel Widths for Calculations of Problem Three

\begin{tabular}{ccccc}
\hline \multirow{2}{*}{$\begin{array}{l}\text { Number of } \\
\text { Channels }\end{array}$} & \multicolumn{3}{c}{ Channel widths } & \multicolumn{2}{l}{ (centimeters) } \\
\cline { 2 - 5 } & $\mathrm{N}=1$ & $\mathrm{~N}=2$ & $\mathrm{~N}=3$ & $\mathrm{~N}=4$ \\
\hline 1 & 30.48 & -- & -- & -- \\
2 & 15.24 & 15.24 & -- & -- \\
3 & 5.715 & 19.05 & 5.715 & -- \\
4 & 5.715 & 9.525 & 9.525 & 5.715 \\
\hline
\end{tabular}


Table 7. Results for Problem Three

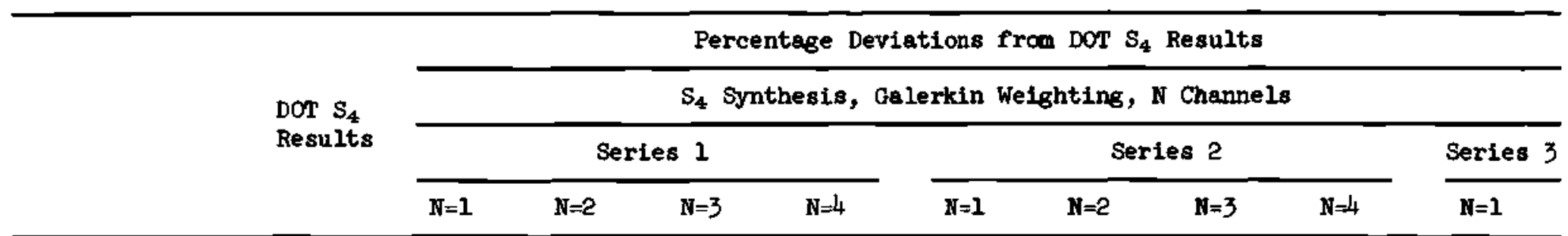

\section{Absorption Rate}

\section{Region}

1

2

3

4

5

6

0.2046
0.2562
0.0403
0.0405
0.1562
0.2046

$+0.73 \%$

-


errors are as large as three per cent. This might be expected, however, since near the zone interface the true flux bears resemblance to both the zones and yet the trial function expansion utilized the base function from one zone only. This is a clear case of inadequate base function selection and, as observed in the previous problems, increasing the number of channels improves the accuracy of the integral results to an acceptable level.

Since the base functions from the two zones are so different and either set alone is inadequate to represent the true flux shape near the zone interface, it is reasonable to ask whether the use of an additional set of base functions, having in some way the characteristics of both zones, would improve the synthesis results. The third set of base functions, obtained from the $S_{4}$ ANISN calculation of an axial homogenization of the two zones, is characteristic of both zones and was used in the third series to facilitate the transition between the two zones. As observed in Table 7, the results for the single channel calculation of the third series are superior to the single channel results for either of the first two series, and are comparable to the four channel results.

Figure 10 shows plots of the horizontal scalar flux near the center of the upper axial zone for the direct DOT calculation and the one and four channel synthesis calculations of series one. Small deviations in the single channel fluxes are noted, but the fluxes from the four channel calculation are practically indistinguishable from the DOT results. Fluxes from series two and three show similar accuracy, as would be expected since at this point on the axis the horizontal 


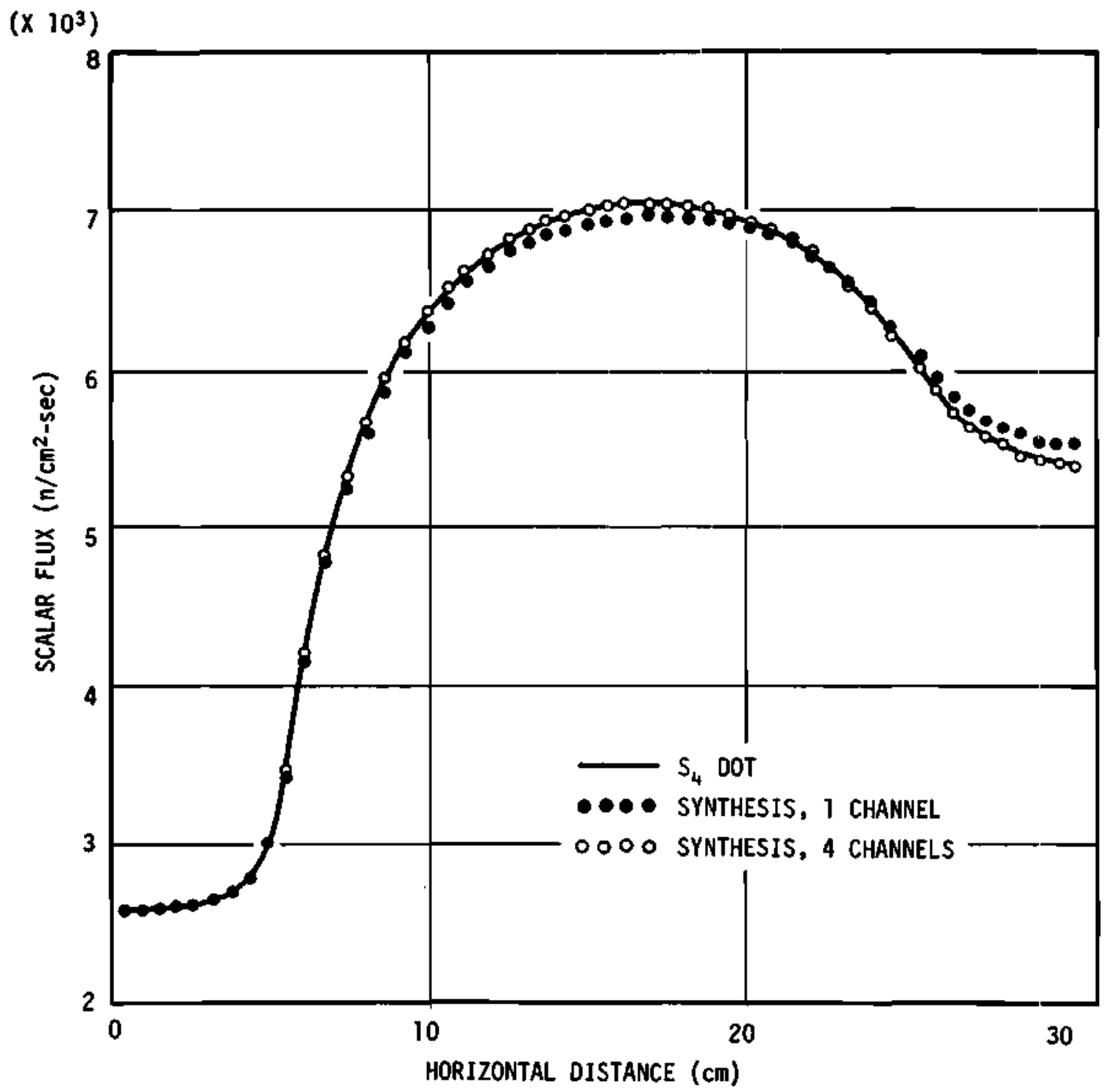

Figure 10. Horizontal Scalar Flux Through the Upper Axial Zone 
scalar flux shape is close to the asymptotic flux shape for the upper zone, and both series includes a set of base functions appropriate to represent that shape.

Near the axial zone interface, however, the horizontal flux shapes are not as accurate. Figure 11 shows a plot of the horizontal fluxes just above the axial zone interface from the DOT calculation, from the one and four channel synthesis calculations of series one and the single channel calculation of series three. The single channel results from series one are seen to be highly inaccurate, but as before, the four channel results are practically indistinguishable from the DOT results. The single channel results from series three, where the "transition" set of base functions was used, are seen to be much more accurate than the single channel results from series one. These results clearly indicate that the use of such a transition set of base functions will allow more accurate results with fewer channels in the synthesis calculation.

Figure 12 shows plots of the horizontal scalar fluxes just above the axial zone interface from the calculations of series two, along with the flux from the DOT calculation. Here the base functions from the upper axial zone are used, and as seen in the single channel plot, are totally inadequate to represent the time flux shape near the interface. Even the four channel plot shows appreciable error in the pointwise results, although the integral results for the four channel case were less than 0.2 per cent in error. Still, however, increasing the number of channels has progressively decreased the pointwise error, at the expense of introducing large discontinuities at the channel 


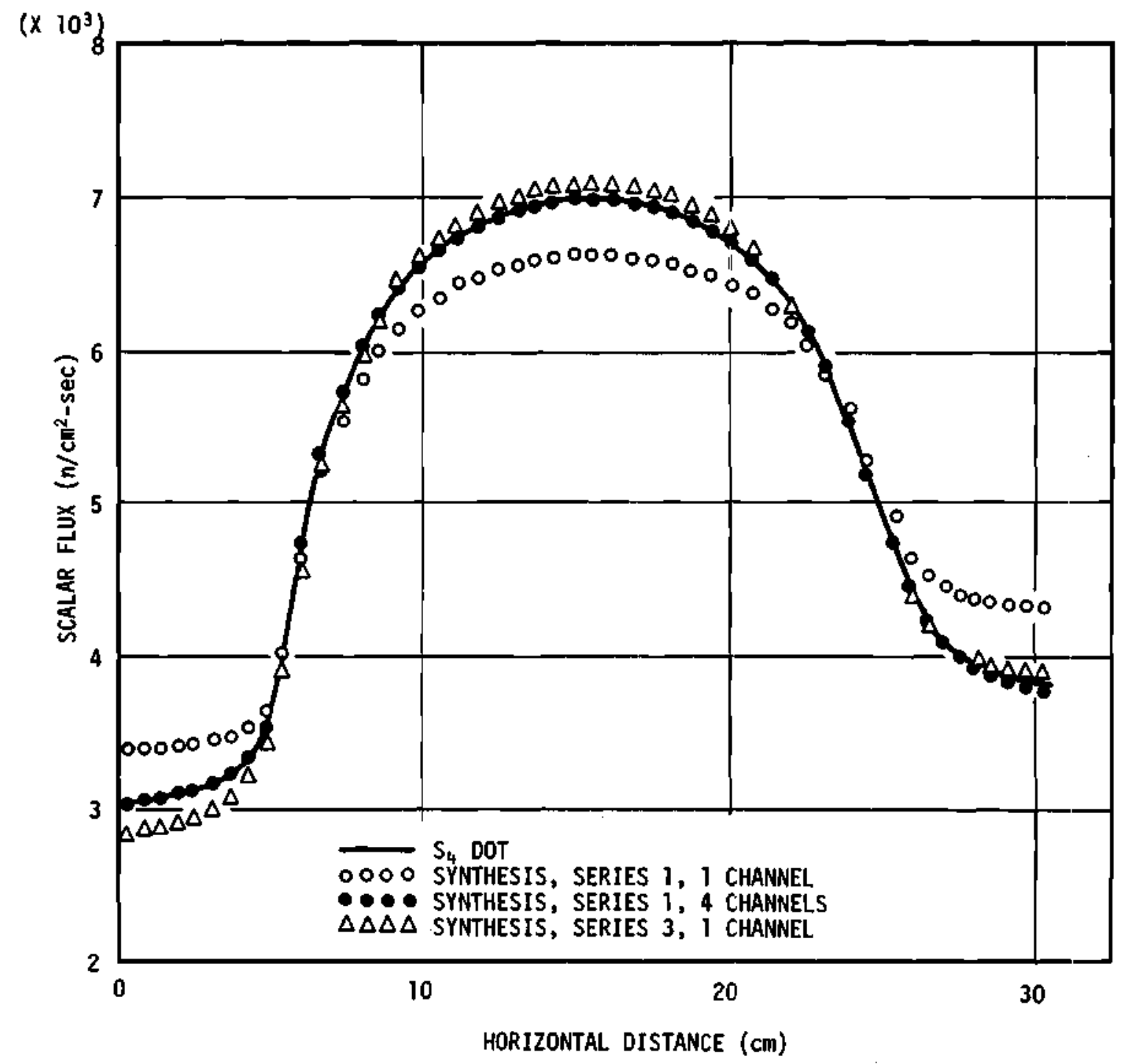

Figure 11. Horizontal Scalar Flux at the Axial Zone Interface, Series One and Three 
(X $\left.10^{3}\right)$

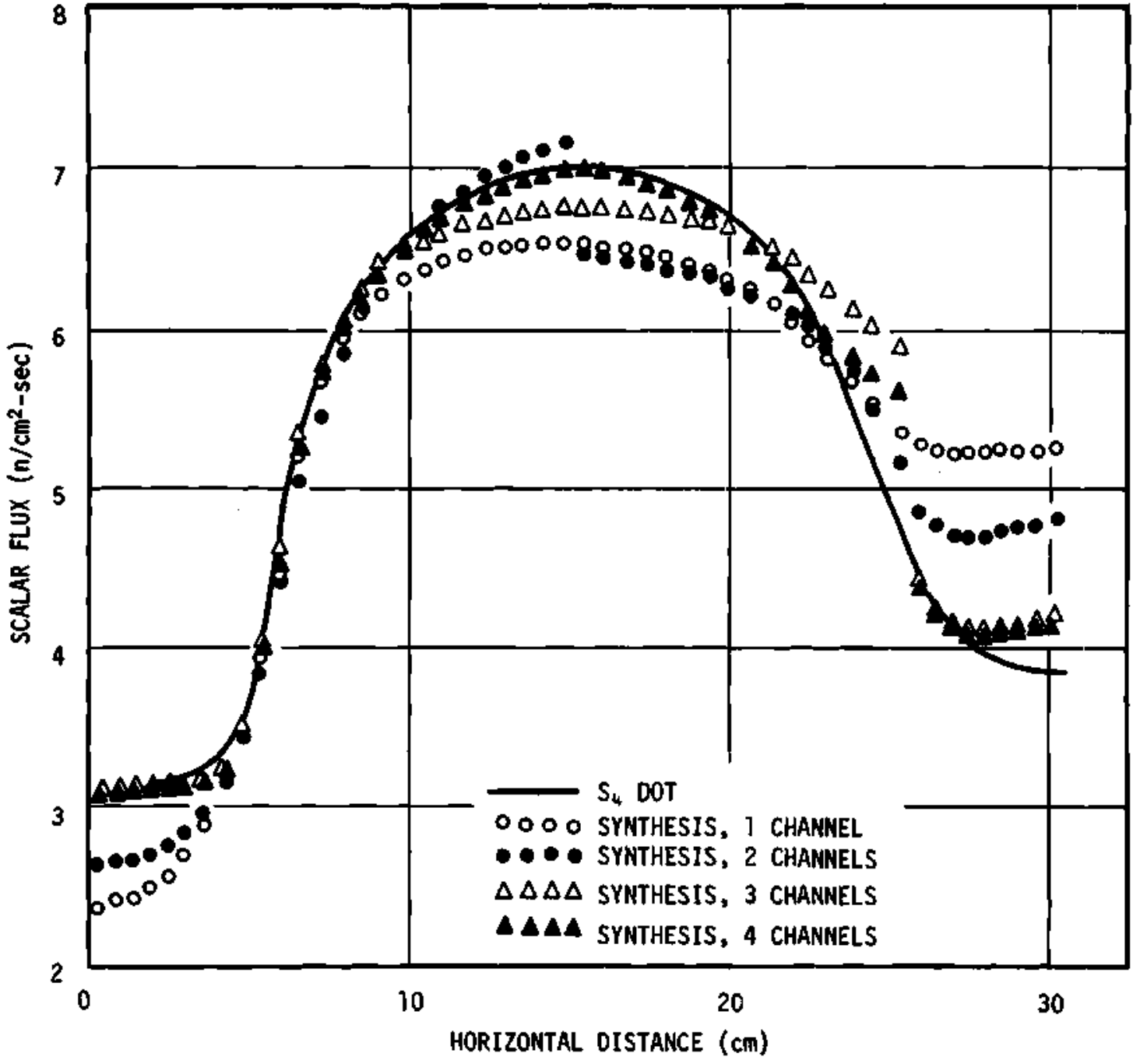

Figure 12. Horizontal Scalar Flux at the Axial Zone Interface, Series Two 
interfaces. Observing this behavior of the synthesized solution, it is not unreasonable to assert that the magnitude of the channel discontinuities may be a useful indication of the adequacy of the base functions and the accuracy of the pointwise results.

The computing times given in Table 7 again show that the synthesis method is capable of producing accurate approximate solutions in less computing time than is required for the direct calculation. Clearly indicated by the comparison of computing times from the three series of synthesis calculations is the fact that the use of a "transition" set of base functions may serve to significantly reduce the cost of an adequate approximate solution.

Problem Four

The fourth problem chosen for calculation by the transport synthesis method is a one-group fixed source problem extracted from the fast group of a four-group cell calculation as shown in Figure 13 . It has been widely studied because of anomalous results observed in the direct finite-difference $S_{n}$ solution to the problem.50,51,23 These anomalous results, called "ray effects", appear as waves in the calculated scalar flux and are a manifestation of the discrete approximation to the continuous angular variable. The nature of the ray effects in general has been studied extensively by Lathrop 51 and several methods have been proposed to alleviate them.43,44 since the synthesis method treats the angular variable in a discrete ordinates approximation, it is reasonable to ask if the ray effects are present in the synthesized solution, and if so, are they similar in nature to those present in the direct discrete ordinates solutions. The variational synthesis 


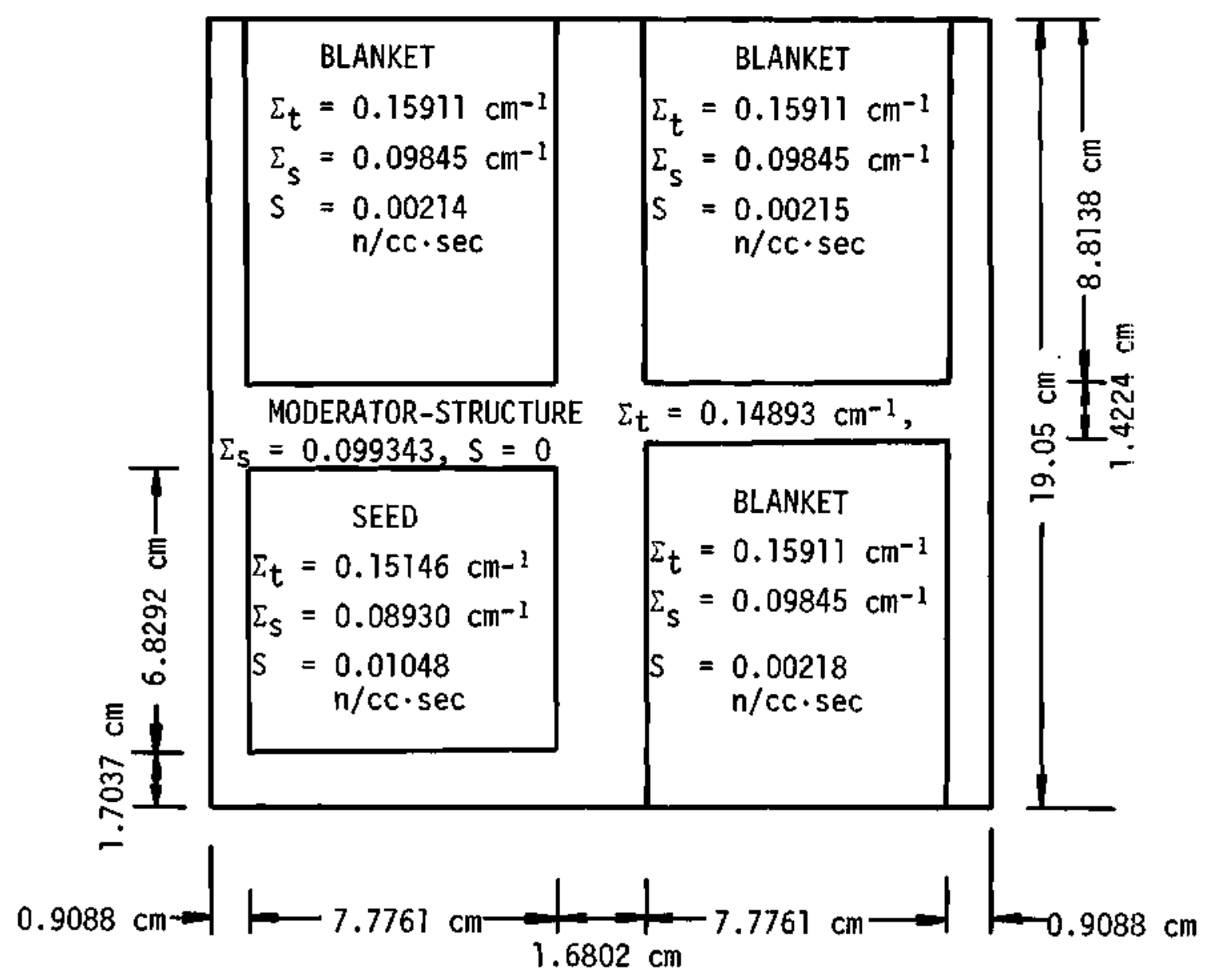

Figure 13. Geometry and Macroscopic Constants for Problem Four 
method has thus been applied to this problem for two reasons: to again demonstrate that it is capable of producing accurate approximate transport solutions when diffusion theory is highly inaccurate and to determine if the ray effects are present in the synthesized solution. To compare the accuracy of the variational synthesis and diffusion-theory solutions, synthesis calculations were made using 12 angular segments to correspond to an $\mathrm{S}_{4}$ angular quadrature. Three sets of base functions were obtained from $S_{4}$ ANISN calculations for use in these synthesis calculations: one from the upper axial zone containing the two blanket regions, one from the axial zone containing the seed region and one blanket region, and one from an axial homogenization of the se two zones.

Table 8 shows the percentage deviations from the DOT resultis in the absorptions by material for three different calculations using the base functions in different ways. Also given for comparison aj:e the percentage deviations in the diffusion-theory results. The first synthesis calculation utilized the first two sets of base functions in all axial zones and a single channel. The integral results are seen to be in excellent agreement with the $S_{4}$ DOT results, while the diffusiontheory results are in error by as much as nine per cent. The running time for the synthesis calculation is seen to be roughly one-seventih that of the finite-difference calculation. The second calculation utilized only one set of base functions in each axial zone. The first set was used in the upper axtal zone and the second set in the other zones. The results for a single channel were poor, since the asymptotic horizontal flux shapes in the two predominant zones are widely different, 
Table 8. Results for Problem Four

\begin{tabular}{|c|c|c|c|c|c|}
\hline & \multirow{3}{*}{$\begin{array}{l}\text { DOT } \mathrm{S}_{4} \\
\text { Results }\end{array}$} & \multicolumn{4}{|c|}{ Percentage Deviations from DOT Results } \\
\hline & & \multicolumn{3}{|c|}{$\mathrm{S}_{4}$ Synthesis, Variational Weighting, N Channels } & \multirow{2}{*}{$\begin{array}{l}\text { Diffusion } \\
\text { Theory }\end{array}$} \\
\hline & & $\underset{N=1}{\text { Calculation } 1}$ & $\underset{N=5}{\text { Calculation } 2}$ & $\underset{N=1}{\text { Calculation } 3}$ & \\
\hline \multicolumn{6}{|l|}{ Absorption Rate } \\
\hline Seed & 0.2592 & $-0.02 \%$ & $-0.38 \%$ & $+0.02 \%$ & $-9.42 \%$ \\
\hline Blanket & 0.4956 & $-0.30 \%$ & $-0.63 \%$ & $-0.24 \%$ & $+3.68 \%$ \\
\hline Moderator-Structure & 0.2451 & $+0.45 \%$ & $+0.22 \%$ & $+0.46 \%$ & $+2 \cdot 50 \%$ \\
\hline $\begin{array}{l}\text { Computing Time } \\
\text { (seconds) }\end{array}$ & 101.0 & $12 \cdot 7$ & 76.3 & 13.9 & 118.0 \\
\hline
\end{tabular}


but increasing the number of channels to five produced acceptable results, as observed in previous problems, at the expense of the running time. The third calculation utilized the third, or "transition," set of base functions in all zones along with the first set in the upper zone and the second set in the lower zones. Again it is seen that the results are quite accurate for a single channel and the running time roughly seven times faster.

Figure 14 shows an axial scalar flux plot through the flux peak in the seed from the $S_{4}$ DOT calculation, the diffusion-theory calculation, and the first synthesis calculation. It is observed that while the synthesis results are very close to the DOT results, the diffusiontheory results are highly inaccurate, particularly near the flux peak. The second and third synthesis calculation produced fluxes with accuracy comparable to the first.

The manifestation of the ray effects for this problem has been studied in detail by Lathrop ${ }^{51}$ and are best seen in his projective plot of the scalar flux from a direct $S_{2}$ discrete ordinates calculation, reproduced in Figure 15. Clearly visible in this plot are ridges in the scalar flux running at 45 degrees through each of the seed and blanket regions. For this particular geometry, the ridges intercept the cell edges at the corners and at the moderator-structure channel and cause the presence of localized maxima. These ridges and localized maxima are the ray effects. For increasing order $S_{n}$ solutions, the prominence of the ridges decreases, as well as the local maxima along the cell edges, and the flux assumes a more smoothly varying shape as would be expected intuitively. For $S_{4}$ or higher quadrature the integral 


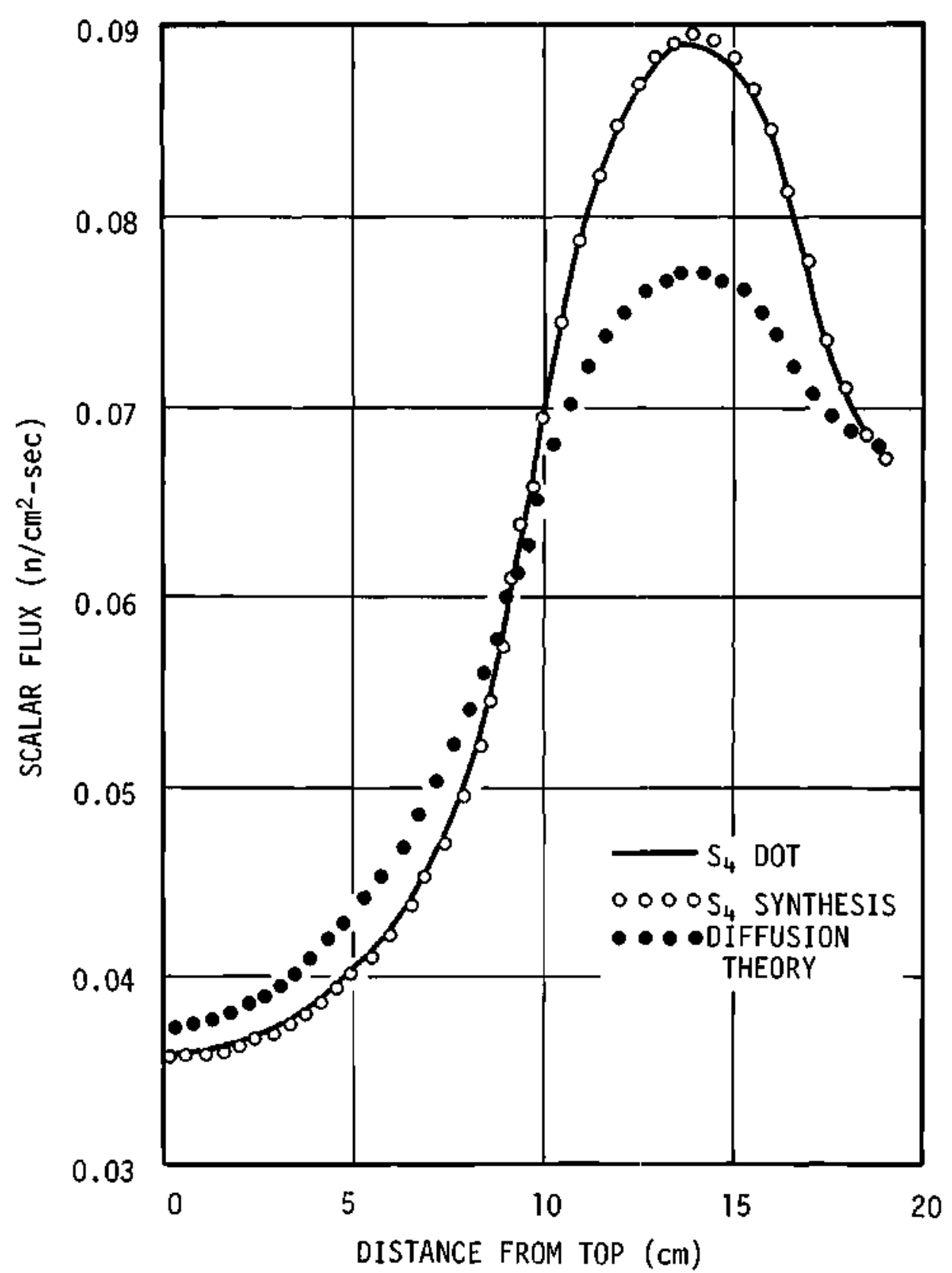

Figure 14. Axial Scalar Flux Through the Seed Region. 


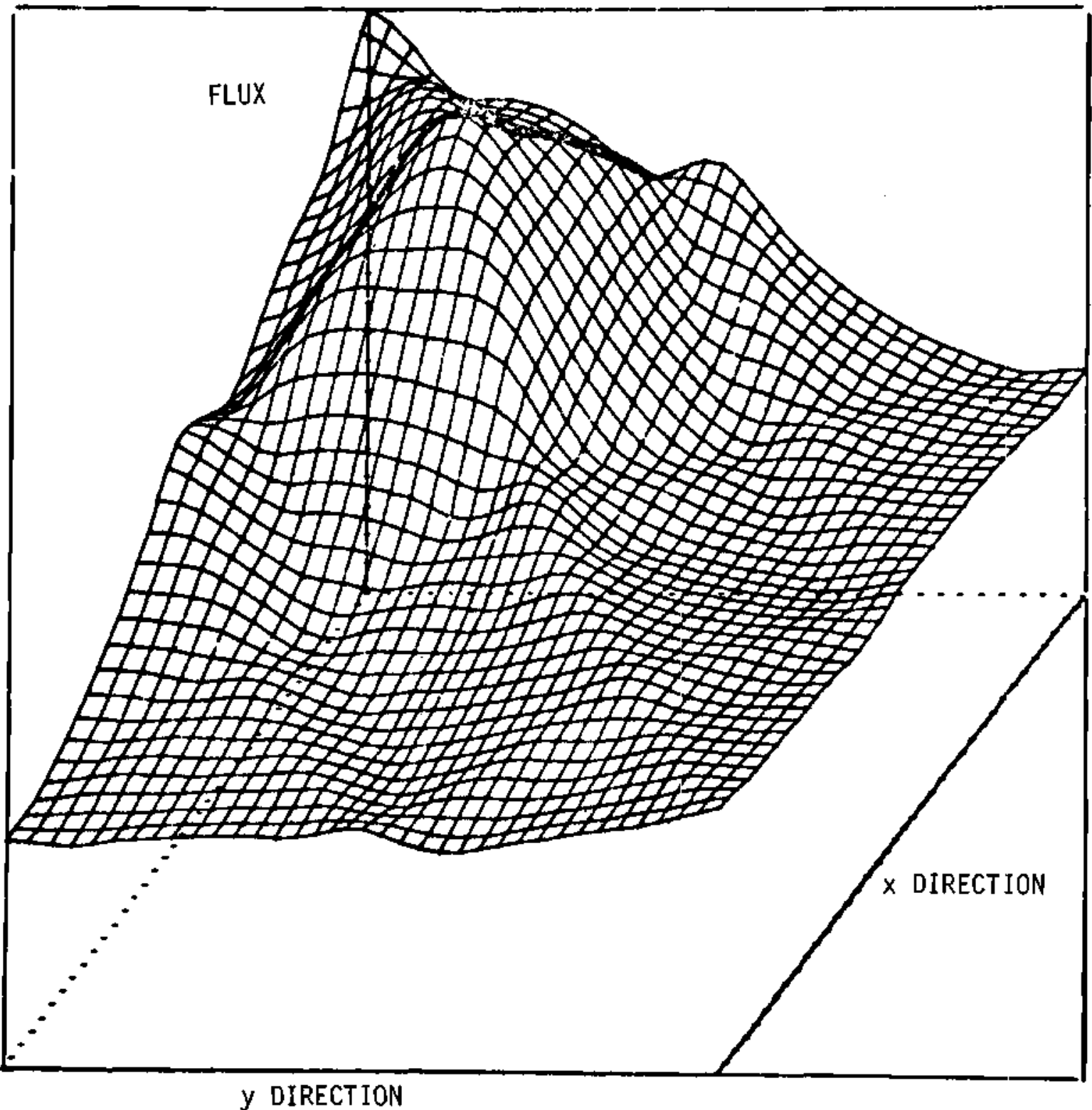

Figure 15. Projective Plot of $S_{2}$ Scalar Flux 
results are only slightly affected by the presence of these local maxima. In fact the $\mathrm{S}_{4}$ integral results differ from the $\mathrm{S}_{10}$ integral results by less than one per cent. 23

Since the ray effects are most severe in the lowest order angular quadrature, the synthesis method has also been used to calculate the problem using four discrete angles, corresponding to an $S_{2}$ angular approximation. Two sets of base functions were obtained from $S_{2} A N I S N$ calculations for each of the two largest axial zones in the cell and both sets were used in all the zones.

Figures 16 and 17 show plots of the soalar flux along the bottom and left edge of the cell for the $S_{2}$ and $S_{4}$ symthesis and Dot calculations. (These correspond, respectively, to the left and back edge fluxes as shown in the projective plot of Figure 15.) The $\mathrm{S}_{4}$ synthesis results given here are from the first $S_{4}$ synthesis calculation described previously.

Along the bottom of the cell, the local maxima observed in the $S_{2}$ and $S_{4}$ DOT results are absent in the $S_{2}$ and $S_{4}$ synthesized fluxes. Along the left edge of the cell, the $S_{2}$ and $S_{4}$ DOT results again exhibit ray effects, while the synthesis results are smoothly varying. In the $\mathrm{S}_{4}$ synthesis results of Figure 17 , a slight wave is seen to be present near the center of the plot. This wave was observed in all the $\mathrm{S}_{4}$ synthesis calculations but is too small to be attributed specifically to ray effects. Comparison of the $s_{2}$ and $s_{4}$ DOT results in this figure also serves to show how increasing the order of angular quadrature decreases the magnitude of the ray effects in the direct solution. 


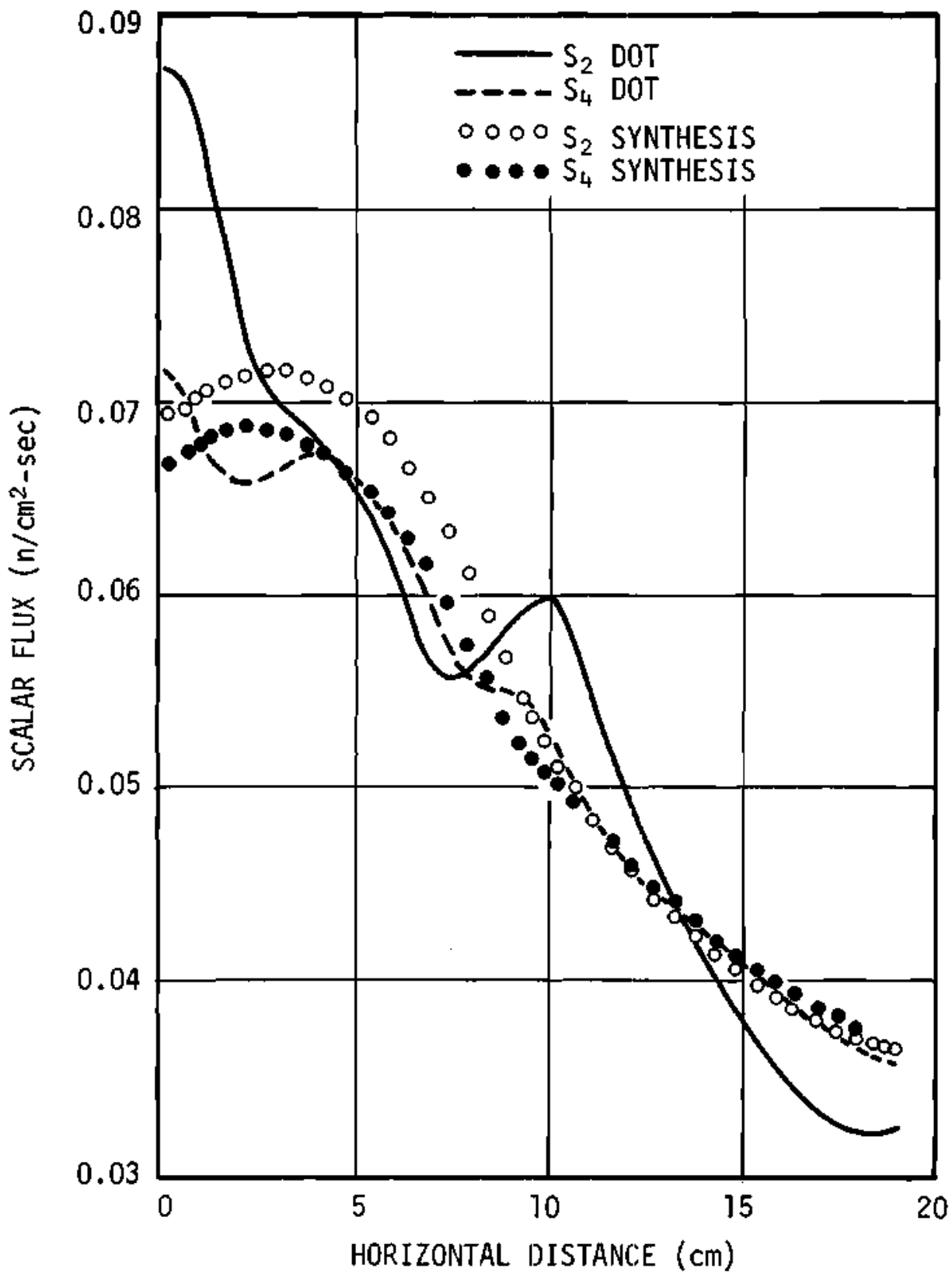

Figure 16. Scalar Fluxes Along Bottom Edge, Problem Four 


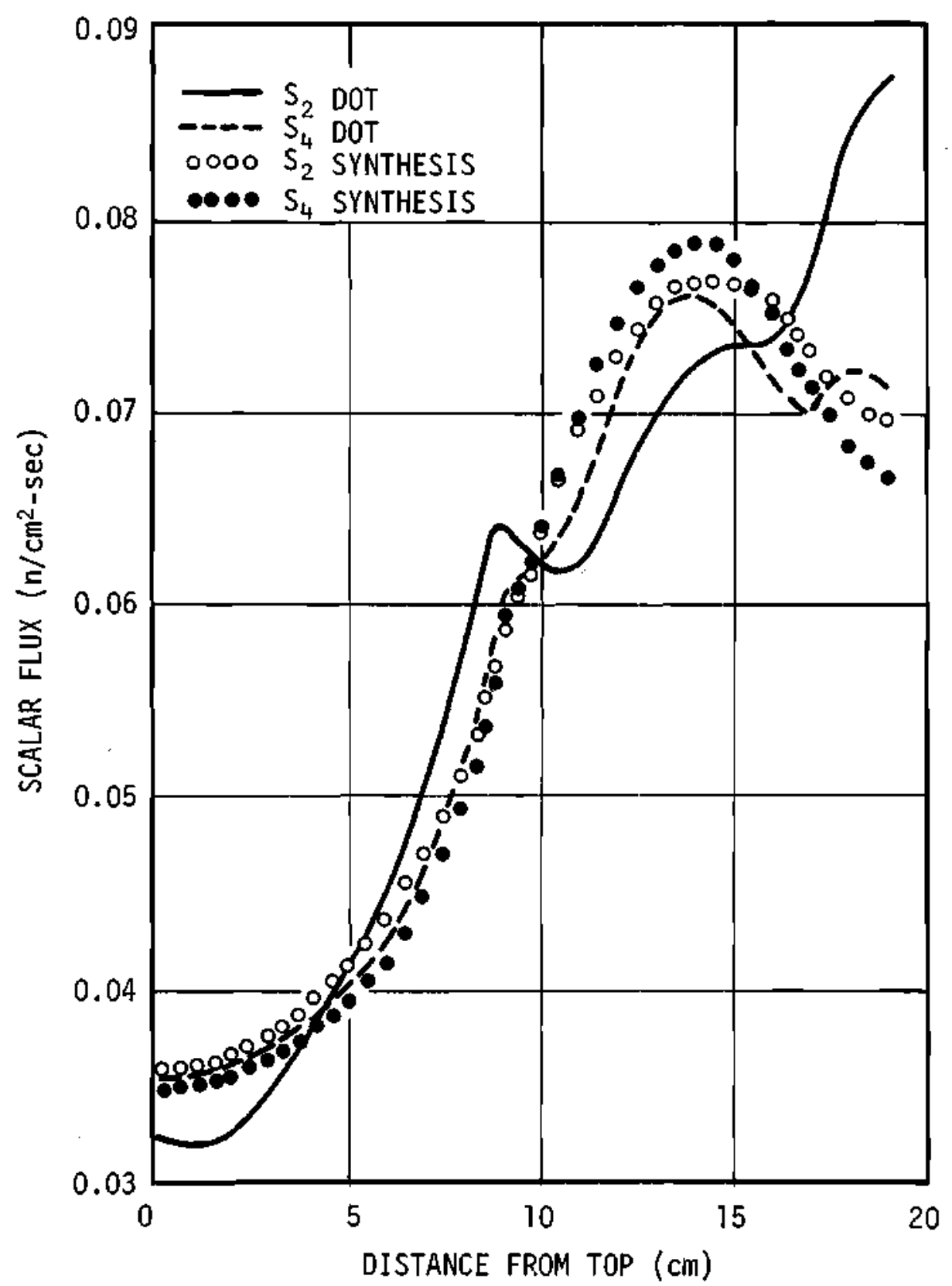

Figure 17. Scalar Fluxes Along Left Edge, Problem Four 
It is important to point out that the results of the synthesis calculations presented here for this problem are similar to results obtained for the same problem by Davis and Kaplan ${ }^{23}$ from the application of a synthesis technique to the discrete ordinates equations. These authors noted from their results that the choice of base functions was not clearly defined and that their approximate solution did not accurately reproduce the ray effects inherent in the direct solution to the discrete ordinates equations which they chose to approkimate. The first point noted has been relieved in the method developed in this work by the inclusion of the multichannel, discontinuous trial function capability. The second point has been clarified in this work through the application of the spatial synthesis approximation to the angular dependent transport equation, whose solution does not exhibit ray effects. The subsequent utilization of a discrete angular segmentation method for obtaining the angular dependent mixing functions in this method has been shown to produce an approximate solution less sensitive to the ray effects. Problem Five

The apparent absence of the appreciable ray effects in the synthesis solution to Problem four has prompted the application of the symthesis method to one further problem. This problem consists of an isolated source in a pure absorber as shown in Figure 18.51 superimposed on the absorber are dashed lines indicating the angles at which the discrete directions in the range $\Omega_{x}<0$ intercept the source edges. In the discrete ordinates approximation, all neutrons moving in directions within an angular segment are approximated as neutrons which 


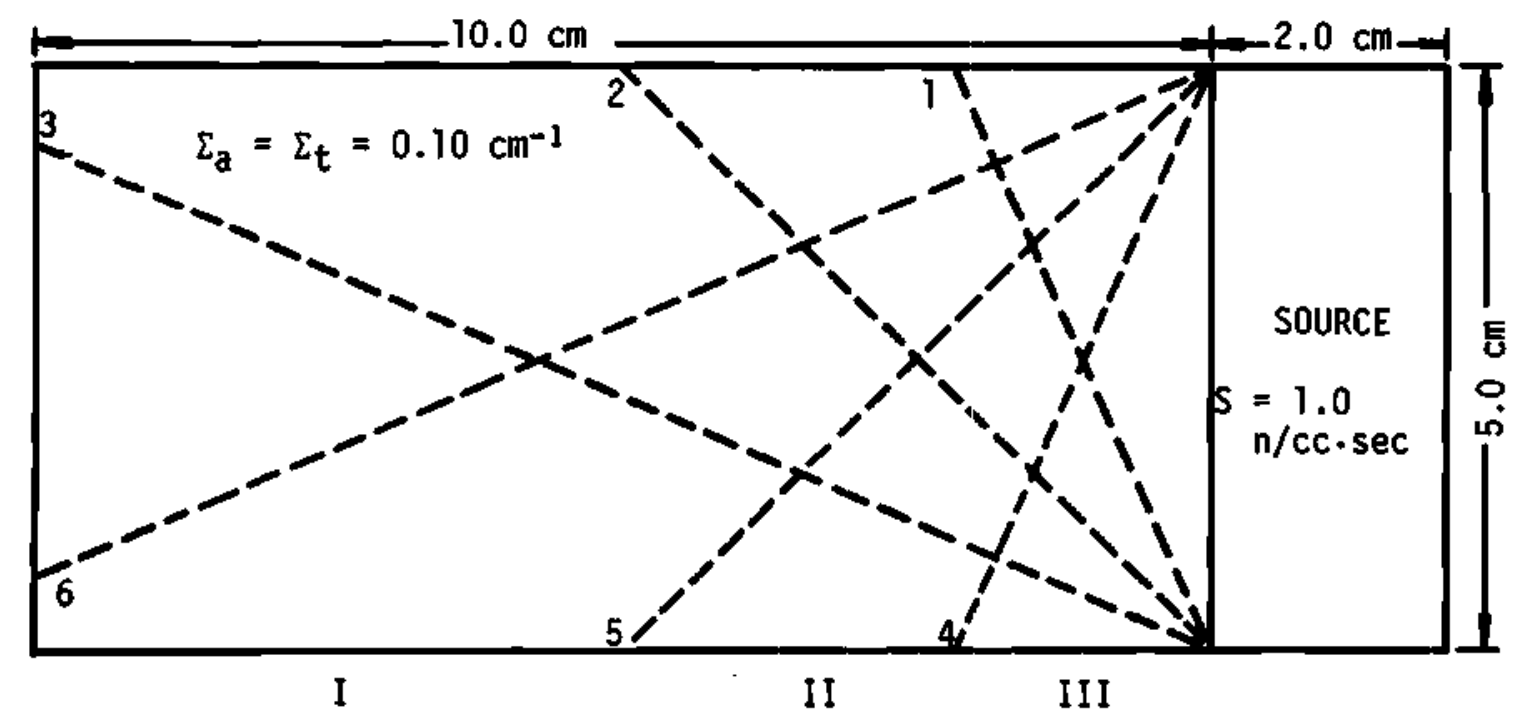

Figure 18. Geometry and Macroscopic Constants for Problem Five 
move in the discrete direction for that segment. Hence it is possible, in situations with isolated sources and no scattering, that neutrons described by a single angle within some segment may fail to "see" an isolated source as a result of the discrete approximation. In the region of the absorber to the left of the dashed line labeled four, for example, the source is not seen by discrete segment four and the angular flux within this segment does not contribute to the scalar flux. At the left edge of the absorber, in the triangular region formed by the lines labeled three and six the source cannot be seen by any directions in the $S_{A}$ quadrature set, and the scalar flux from a direct $S_{4}$ calculation would be expected to be zero within this region. This is an extreme case of inaccuracies due to ray effects, which in the absence of scattering sources is primarily a geometrical effect.

The variational synthesis method has been used to calculate this problem in an $S_{4}$ angular approximation using base functions from $\mathrm{S}_{4}$ ANISN calculation. An $\mathrm{S}_{4}$ DOT calculation wes also performed for comparison. Figure 19 shows a plot of the scalar flux along the left edge of the absorber from the DOT and synthesis calculations. It is seen that the scalar flux from the DOT calculation fall rapidly to zero within the triangular region and that the same type behavior is present in the synthesized solution. For the synthesized solution, however, the flux dip is less evere.

Figure 20 shows the scalar fluxes along the bottom edge of the absorber. The DOT solution is seen to exhibit waves corresponding to the three regions indicated along the $x$-axis and on the geometry in Figure 18. In region one the source is seen by only one direction; in 


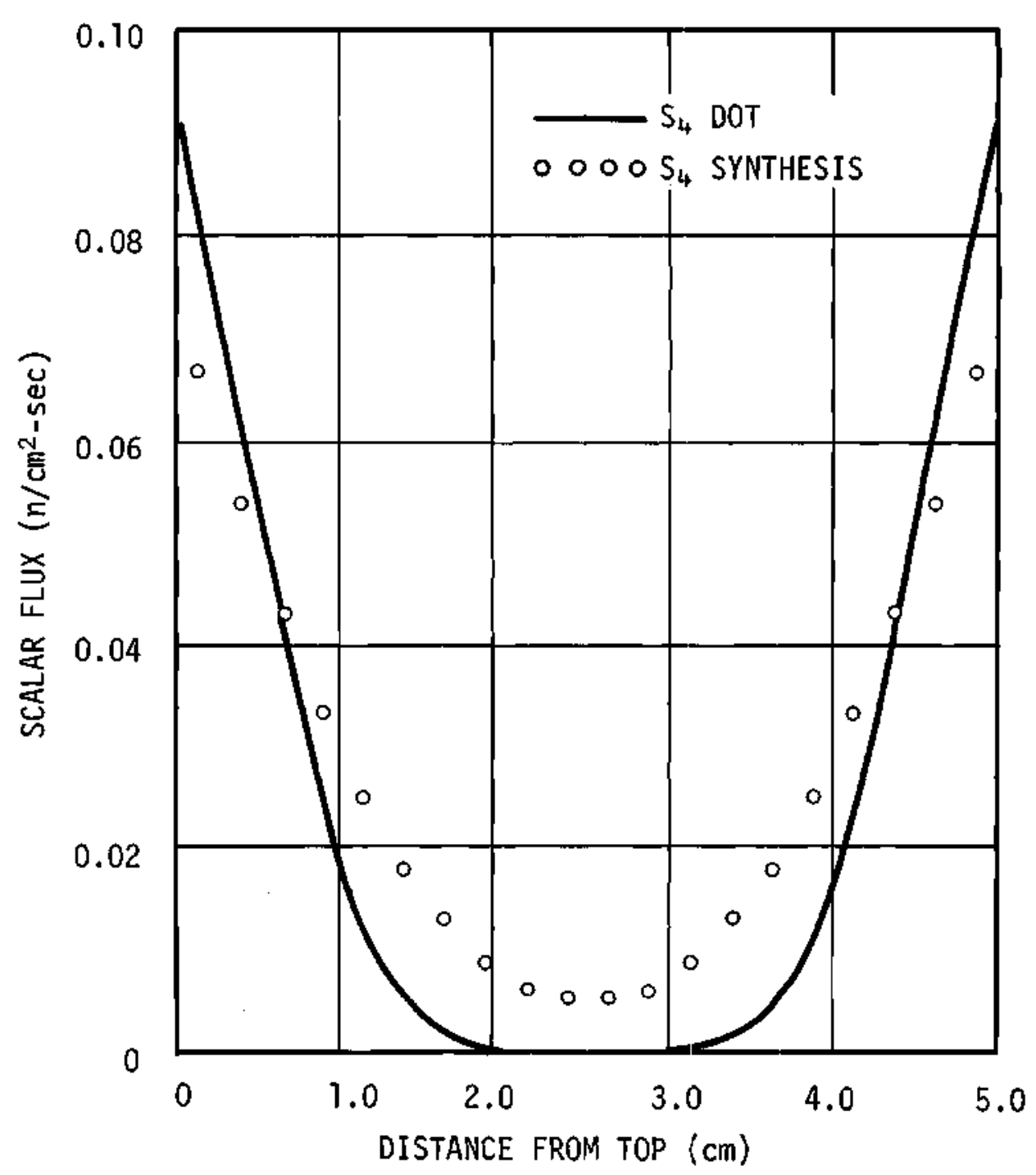

Figure 19. Scalar Flux Along Left Edge, Problem Five 


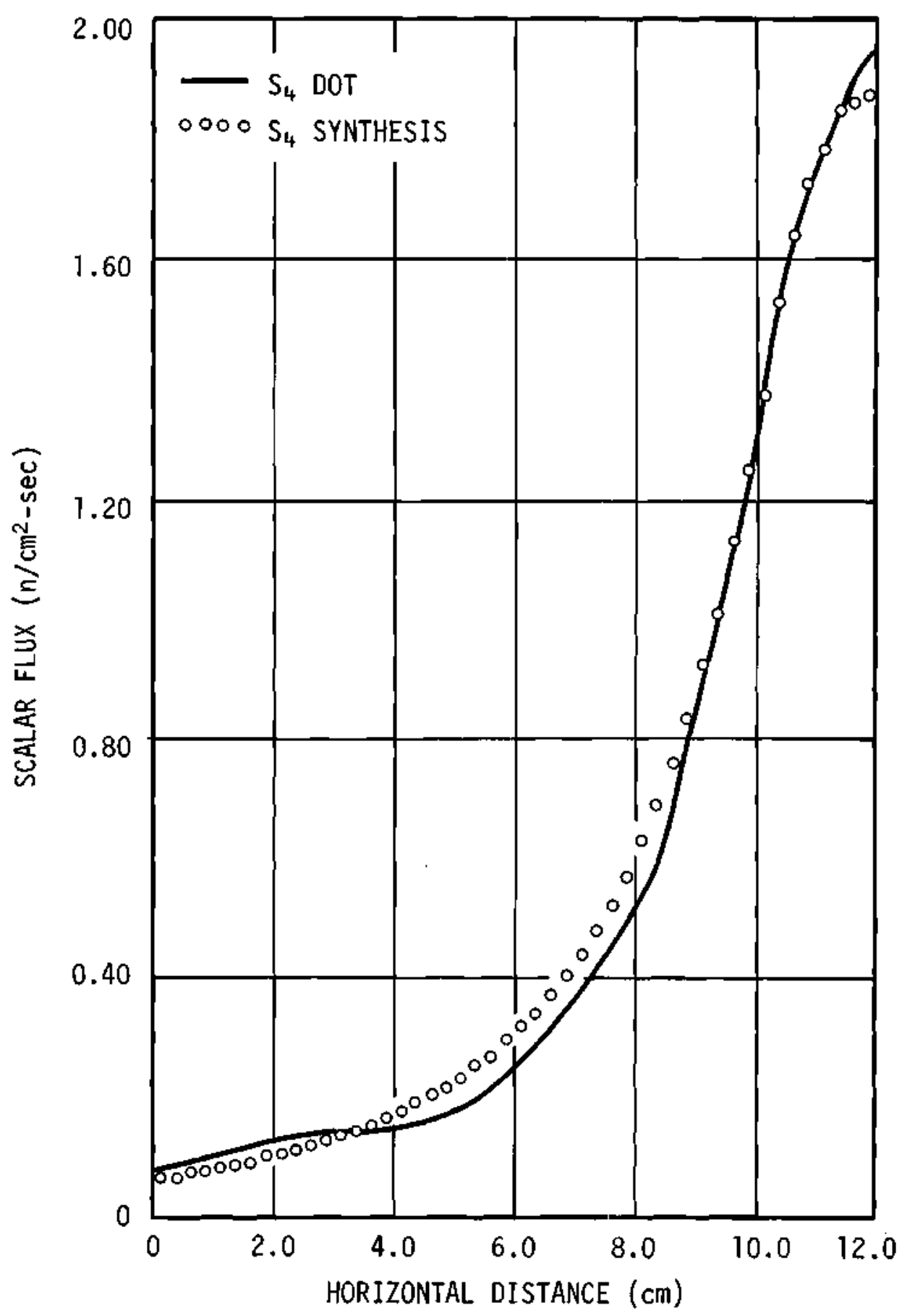

Figure 20. Scalar Flux Along Bottom Edge, Problem Five 
region two by two directions and in region three by three directions. These, too, are the ray effects. The synthesis solution, however, does not exhibit these waves and is a smoothly varying curve as would be expected since trial function expansion has assumed the $\mathrm{x}$-variation of the flux to be the sum of base functions which are smooth exponentials. This problem thus serves to indicate that, in the extreme case, the synthesis solution will exhibit ray effects along the remaining spatial variable which are similar to, but less severe than, those present in the direct discrete ordinates solution. Along the base function axis, however, the shape of the synthesized solution has been assumed in the trial function and the ray effects will not appear. 


\section{CHAPTER VI}

\section{CONCLUSIONS}

The variational synthesis method has been applied to the steadystate Boltzmann neutron transport equation in two- and three-dimensional rectangular geometry. In this application the energy dependence has been treated in the standard multigroup energy approximation, the dependence along all but a single remaining spatial variable has been treated by a multichannel discontinuous spatial synthesis approximation and the angular dependence has been treated by a discrete ordinates approximation. All approximate treatments of these variables have been developed consistently from a variational principle by the standard trial function reduction procedure, applied sequentially to the energy variable, the spatial variables and the angular variable. The approximate method as developed in this application allows the user to obtain approximate solutions to the transport equation by combination of the solutions to related problems of lower dimensionality.

An experimental computer code, the TS2 code, has been written by the author for use in obtaining approximate solution to the twodimensional, one-energy-group problem with isotropic scattering. The TS2 code has been used to obtain approximate solutions to several test problems of the type encountered in the reactor field and the accuracy and comparative cost of these approximate solutions has been determined by comparison to consistently obtained direct solutions to the same: 
problems. Both fixed source and eigenvalue problems, as well as problems with both vacuum and reflected boundary conditions, have been investigated.

The variational synthesis method utilizing a continuous trial function approximation and a well chosen set of base functions has been shown capable of producing integral results within one per cent of the results of direct discrete ordinates calculations in situations where diffusion theory results are in error by 25 per cent or more. The ability of the synthesis method to accurately approximate the steep scalar flux gradients in situations where the diffusion approximation is invalid has also been demonstrated. The comparative cost of the approximate continuous synthesis solution has been shown to be of the same order as the cost of the diffusion theory solution and as little as one-sixth of the cost of the direct discrete ordinates transport solution.

The variational synthesis method utilizing the multichannel discontinuous trial function approximation, which has proven useful in applications of the symthesis method to diffusion theory problems, has been included in this application. The usefulness of the multichannel technique in situations where the choice of base functions is not clearly defined has been demonstrated by obtaining approximate synthesis solutions using base functions which were poorly chosen. In these solutions, where a continuous single channel symthesis technique produced errors in integral results by as much as ten per cent, the iniroduction of the multichannel technique has been shown to increase the accuracy of the approximate solution as the number of channels in 
increased, yielding errors as small as one-half per cent, or less. The cost of this increased accuracy is an associated increase in the computing time required to obtain the approximate solution. While the multichannel technique is capable of producing accurate integral results when very poor base functions are used, the pointwise accuracy of the synthesized scalar flux resulting from very poor base functions has been shown to be appreciably in error. In these situations, the pointwise solutions exhibit large discontinuities at the channel interfaces. Use of the multichannel technique with a well chosen set of base functions has been shown capable of producing integral results with accuracy of the order of level of convergence, and pointwise results practically indistinguishable from the results of the direct transport solution. Noting this behavior, the observation of the discontinutties in the resultant synthesized solution has been proposed as a qualitative measure of the pointwise accuracy and the adequacy of the base functions used in obtaining the solution.

The ability to use particular base functions only over the range of the remaining spatial variable where they are most representative of the true solution has also been included in this application. Resilts from test problems where base functions were switched have shown that, in the case where all base functions were switched at the same point, results were somewhat inferior to the results obtained when the base functions were not switched. In cases where only certain of the base functions were switched at a single polnt, results have shown that superior accuracy may be obtained by employing this technique, parcicularly if a special set of "transition" base functions is used. While 
the applicability of the base function switching technique has been demonstrated, its full usefulness should become more apparent in applications to large scale problems having many axial zones, where the range of the remaining spatial variable covers many neutron mean free paths.

The use of the Galerkin weighting method has been shown to produce errors not significantly greater than the variational weighting method. In larger scale problems, this fact will significantly reduce the cost of approximation by allowing the reactor designer to avoli the lower dimensional adjoint solutions necessary to obtain adjoint base functions for use in the variational weighting method.

The generality of the angular treatment in this application has been demonstrated by obtaining synthesis solutions using different angular quadrature for some of the test problems. The results indicate that the synthesis solution for a given angular quadrature, at least in the integral sense, should be expected to approximate the results of a direct discrete ordinates solution using the same quadrature. The same indications are apparent in the pointwise results for problems where sources are not isolated and where ray effects are not present to a great degree in the direct discrete ordinates solution. In situations where the ray effects are present, the pointwise results from the synthesis solution have been shown to approximate the discrete ordinates solution of the same angular quadrature in an overall sense, with the localized maxima and waves of the ray effects being effectively smoothed in the synthesis solution. In a very extreme example, the synthesis solution has been shown to exhibit ray effects similar to, but less 
severe than, those present in the direct solution. For this reason, use of the synthesis method is not proposed as a technique solely for avolding ray effects.

The purposes of this research as adopted in Chapter I have been realized. A variational synthesis technique, utilizing the multichannel discontinuous trial function capability, has been developed for obtaining approximate two- and three-dimensional transport solutions. The development of the experimental TS2 code and its use in obtaining accurate approximate synthesis solutions at a fraction of the cost of the direct solutions, and with accuracy superior to the diffusion approximation has demonstrated the applicability of the method to neutron transport problems in reactor physics. 
CHAPTER VII

\section{RECOMMENDATIONS}

Several refinements in the application of the variational symthesis method as developed in this work are immediately obvious. Most important among these are the incorporation of more than a single energy group and the determination of the usual eigenvalue, $k$ eff' in the general case rather than the elgenvalue, $\frac{1}{c}$, as determined by the TS2 code. The necessity for the inclusion of high order anisotropic scattering in an approximate method of this type is certainly open to question. It is felt that isotropic scattering with a transport corrected total cross section would most likely be adequate for a great majority of problems to which the method might be applied. Refine.. ments to the approximate technique, serving to increase its generality, might be to include the capability of using different numbers of betse functions in different axial zones and in different energy groups. Both of these refinements could be incorporated consistently in the variational development and, while increasing the notational complexity, should prove useful in the application to large scale problems. The method employed to obtain the two-dimensional solutions in the TS2 code should be applied to obtain an actual three-dimensional solution. Modifications would consist basically of those necessary to the routines calculating the matrix synthesis constants, and to the iteration routines allowing additional discrete angles to be considered as required for the three-dimensional problem. A natural extension of 
the method would be the inclusion of geometries other than rectangular geometry. For the two-dimensional cylindrical geometry $(r, z)$ case the extension is minor if the spatial variable removed by the synthesis approximation is the z-axis. The resulting equations for the discrete angular mixing functions $\phi_{\text {gnd }}(r)$ would be identical to those of the two-dimensional rectangular geometry case except for the addition of a single term representing the angular derivative and serving as additional coupling between the discrete segments. For other curved geometries, $(r, \theta)$ for example, the use of the technique developed here would be more difflcult due to coupling between discrete segments in different angular ranges, for which different base functions are used. Regarding the choice of base functions, the most frequent criticism heard in discussions of any synthesis method, only experience with the method over a wide class of problems will serve to delineate standard criteria for their selection. 
124

APPENDICES 
APPENDIX A

\section{A FUNCTIONAL FOR THE VARIATIONAL CHARACTERIZATION OF THE NEUTRON TRANSPORT PROBLEM}

In order to obtain a variational characterization of the transport equation, a functional must be constructed whose suler equations are the forward and adjoint transport equations and their associated boundary and continuity conditions. That is, a functional $\mathscr{\Re}\left[\phi, \phi^{*}\right]$ is sought, such that the variational principle

$$
8 \mathscr{F}=0
$$

is equivalent to the statement that $\phi$ and $\phi^{*}$ are solutions to the forward and adjoint transport equations. The adjoint solution must be included in this development, of course, since the operators in the transport equation are not self-adjoint. Consider the transport equation in operator form:

$$
T \Psi=S
$$

where $\Psi$ is the neutron flux density in the space-angle-energy phase space, $T$ is the transport operator which takes into account the effects of scattering, absorption and spatial movement of neutrons, $s$ is the rate per unit phase space volume at which neutrons are introduced into the system.

The method chosen here to construct the desired functional is to use a functional of the Roussopoulos type for the transport equation 
itself and to include the boundary and continuity conditions by the use of Lagrange multipliers. This development closely parallels a similar development as given by O'Reilly. ${ }^{26}$ The Roussopoulos functional has the form:

$$
\mathscr{F}\left[\phi, \phi^{*}\right]=\int_{\text {Phase }} \mathrm{dV}\left\{\phi^{*}[\mathrm{~S}-\mathrm{T} \phi]+\mathrm{S}^{*} \phi\right\}
$$

where $\phi$ and $\phi^{*}$ are "arbitrary" functions of the phase space coordinates which satisfy the same boundary and continuity conditions as those imposed on the solutions of the forward and adjoint transport equation. $S^{*}$ is the source in the adjoint transport equation and is usually chosen as some reaction rate probability of interest.

To show that the Roussopoulos functional is an appropriate functional for the transport equation, it is necessary to show that the functional is stationary when $\phi$ and $\phi^{*}$ are the solutions to the transport equations. First let $\phi^{*}$ be varied by an amount $\epsilon^{*} \eta^{*}$, where $\epsilon^{*}$ is an independent parameter and $\eta^{*}$ is an arbitrary function of the phase space coordinates which vanishes on the phase space boundaries. The new value of the functional as a result of this variation is

$$
F\left[\phi, \phi^{*}+\epsilon^{*} \eta^{*}\right]=\int_{\text {Phase Space }} \mathrm{dV}\left\{\left(\phi^{*}+\epsilon^{*} \eta^{*}\right)[S-\mathrm{T} \phi]+S^{*} \phi\right\}
$$

If the functional is stationary, then the first variation of with respect to $\phi^{*}$, denoted by $8 \mathcal{F}_{\phi^{*}}$, must vanish:

$$
\delta \mathscr{F}_{\phi^{*}}=\lim _{\epsilon^{*} \rightarrow 0} \frac{\partial}{\partial \epsilon^{*}} \mathscr{F}\left[\phi, \phi^{*}+\epsilon^{*} \eta^{*}\right]
$$


or

$$
8 \xi_{\phi^{*}}=\int_{\text {Phase Space }} d V \eta^{*}[S-T \phi]=0
$$

It follows from a typical fundamental lemma of the calculus of variation that Equation A.5b is satisfied only if the coefficient of $T^{*}$ vanishes identically, thus requiring that

$$
T \phi=S
$$

This requirement is met only if $\phi$ satisfies the transport equation, 1.e.,

$$
\phi=\Psi
$$

Using the usual definition of an adjoint operator $\mathrm{T}^{*}$,

$$
\underset{\text { Phase Space }}{\int \mathrm{dV} \phi * T \phi}=\underset{\text { Phase Space }}{\int \mathrm{dV} \phi \mathrm{T}^{*} \phi *}
$$

Equation A.3 can be rewritten as

$$
\mathscr{F}\left[\phi, \phi^{*}\right]=\int_{\text {Phase Space }} \mathrm{dV}\left\{\phi\left[\mathrm{S}^{*}-\mathrm{T}^{*} \phi^{*}\right]+S \phi^{*}\right\}
$$

Now let $\phi$ be varied by an amount $\epsilon \eta$ in Equation $A .9$, where $\epsilon$ is an independent parameter, and $\eta$ is an arbitrary function of the phase space coordinates which vanishes at the boundaries. The value of the functional as a result of this variation is

$$
\mathscr{F}\left[\phi+\epsilon \phi_{T}, \phi^{*}\right]=\int_{\text {Phase Space }} \mathrm{dV}\left\{(\phi+\epsilon \eta)\left[S^{*}-\mathrm{T}^{*} \phi^{*}\right]+S \phi^{*}\right\}
$$


If the functional is stationary then the first variation with respect to $\phi, \delta F_{\phi}$, must also vanish:

$$
\delta \mathcal{F}_{\phi}=\lim _{\epsilon \rightarrow 0} \frac{\partial}{\partial \epsilon} \mathscr{F}\left[\phi+\epsilon \eta, \phi^{*}\right]=0
$$

or

$$
\delta \xi_{\phi}=\int_{\text {Phase Space }} \mathrm{dV} \eta\left[\mathrm{S}^{*}-\mathrm{T}^{*} \phi^{*}\right]=0
$$

Again, by the same lemma, Equation A.Ilb is satisfied only if the coefficient of $\eta$ vanishes, thereby requiring

$$
T^{*} \phi^{*}=S^{*}
$$

Equation A.12 is, of course, the adjoint transport equation, hence, at the stationary point,

$$
\phi^{*}=\Psi^{*}
$$

It is thus seen that within the class of the functions $\phi$ and $\phi^{*}$ satisfying the same boundary and continuity conditions as those imposed on the solutions to the forward and adjoint transport equations, the Roussopoulos functional $\left[\phi, \phi^{*}\right]$ is stationary when

$$
\phi=\Psi
$$

and

$$
\phi^{*}=\Psi^{*}
$$

hence the variational principle 


$$
8 \%=0
$$

is an appropriate variational characterization of the transport equation within this class of functions.

\section{Discontinuous Trial Functions}

When using the variational method as an approximative techiniqu, it is often desirable to remove the condition that the functions $\phi$ and $\phi^{*}$ forming the domain of $F$ satisfy the same boundary and continuity conditions as the forward and adjoint neutron fluxes. In particular it is desirable to extend the domain so as to include functions which are only piecewise continuous and do not satisfy the boundary and continuity conditions.

This extension can be accomplished by adding the boundary conditions and continuity conditions to the functional F with the use of Lagrange multipliers. This extension is developed more clearly by writing the transport equation explicitly and by considering a definite two region geometry as shown in Figure 2l. The generalization to a multiregion structure will then follow directly from the two-region case.

The Two-Region Reactor

Consider a finite volumetric region $R$ enclosed by a convex surface $s$, arbitrarily subdivided into two subregions $\Re_{1}$ and $\Re_{2}$ by the interior surface $\sigma$. Assuming neutrons arise within $\Re$ only from a source distribution $S(\vec{r}, \hat{\Omega}, E)$, the transport equation can be written 


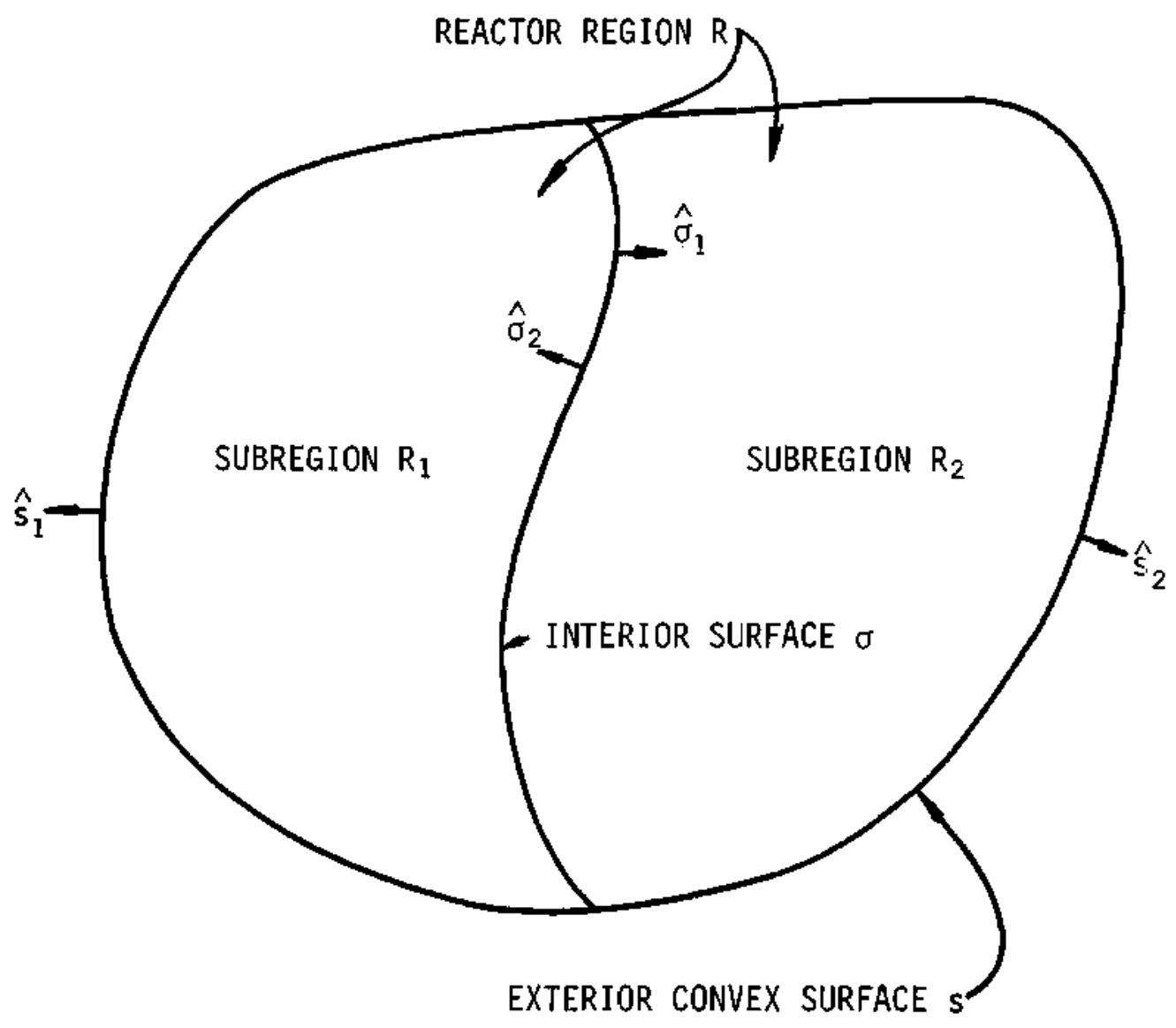

Figure 21. Convex, Two-Region Reactor Geometry 


$$
\begin{gathered}
{\left[\hat{\Omega} \cdot \nabla+\Sigma_{t}(\vec{r}, E)\right] \Psi(\vec{r}, \hat{\Omega}, E)=\int_{0}^{\infty} d E^{\prime} \iint_{4 \pi} d \hat{\Omega}^{\prime} \Sigma_{s}\left(\vec{r}, \hat{\Omega}, \hat{\Omega}^{\prime}, E, E^{\prime}\right) \Psi\left(\vec{r}, \hat{\Omega}^{\prime}, E^{\prime}\right)} \\
+S(\vec{r}, \hat{\Omega}, E)
\end{gathered}
$$

where the notation used is the same as defined in Chapter III. The boundary and continuity conditions necessary to complete the description of neutron migration within $\mathbb{R}$ are:

(1) No neutrons enter $R$ through the external surface $s$.

(2) The neutron flux is spatially continuous across the interior surface $\sigma$. These conditions can be written mathematically as

$$
\Psi(\vec{R}, \hat{\Omega}, E)=0, \hat{\Omega} \cdot \hat{s}<0
$$

and

$$
\Psi\left(\vec{r}_{\sigma_{2}}, \hat{\Omega}, E\right)=\Psi\left(\vec{r}_{\sigma_{2}}, \hat{\Omega}, E\right)
$$

where $\vec{R}$ represents points along the exterior surface $s$, and $\hat{s}$ is the outward unit normel to s. $\vec{r}_{\sigma_{I}}$ and $\vec{r}_{\sigma_{2}}$ represent points along the interior surface $\sigma$ as approached from subregions $\mathfrak{R}_{1}$ and $\mathscr{R}_{2}$, respectj.vely. In further discussion it is also convenient to let $\hat{\sigma}_{I}$ and $\hat{\sigma}_{2}$ denote the unit normals to the interior surface $\sigma$ which point away from $\Re_{1}$ anc. $\mathscr{R}_{2}$, respectively.

Adding the conditions A.17 and A.18 to the Roussopoulos functional in Equation A.3 by the use of Lagrange multipliers gives the following functional: 


$$
\begin{aligned}
& \mathscr{F}\left[\phi, \phi^{*}, \alpha, \beta\right]=\left(\iiint_{\mathfrak{R}_{1}} \mathrm{~d} \vec{r}+\iiint_{\mathbb{R}_{2}} \mathrm{~d} \vec{r}\right) \iint_{4} \mathrm{~d} \hat{\Omega} \int_{0}^{\infty} \mathrm{dE}\left\{\phi^{*}[\mathrm{~S}\right. \\
& \left.\left.-\iint_{4 \pi} \mathrm{d} \hat{\Omega} \int_{0}^{\infty} \mathrm{dE}^{\prime} \Sigma_{s}\left(\vec{r}, \hat{\Omega}, \hat{\Omega}^{\prime}, E, E^{\prime}\right) \phi\left(\vec{r}, \hat{\Omega}^{\prime}, E^{\prime}\right)-\hat{\Omega} \cdot \nabla \phi-\Sigma_{t} \phi\right]+S^{*} \phi\right\} \\
& +\iint_{\sigma} \mathrm{d} \sigma \int_{4 \pi} \mathrm{d} \hat{\Omega} \int_{0}^{\infty} \mathrm{dE} \alpha\left(\vec{r}_{\sigma}, \hat{\Omega}, E\right)\left[\hat{\sigma}_{1} \cdot \hat{\Omega} \phi\left(\vec{r}_{\sigma_{\lambda}}, \hat{\Omega}, E\right)+\hat{\sigma}_{2} \cdot \hat{\Omega} \phi\left(\vec{r}_{\sigma_{2}}, \hat{\Omega}, \mathrm{J}\right)\right] \\
& +\iint_{S} d s \iint_{4 \pi} d \hat{\Omega} H(-\hat{\Omega} \cdot \hat{s}) \int_{0}^{\infty} d E \beta(\vec{R}, \hat{\Omega}, E) \hat{\Omega} \cdot \hat{s} \phi(\vec{R}, \hat{\Omega}, E)
\end{aligned}
$$

where $\alpha$ and $\beta$ are the as yet undetermined Lagrange multipliers. In that the continuity condition is to be imposed at every point along the interior surface $\sigma$, for all values of angle and energy, the multiplier, $\alpha$, must be a function of $\vec{r}_{\sigma}, \hat{\Omega}$ and $E$ and the sum of all constraint conditions becomes an integral over angle, energy and the surface $\sigma$. Similarly, the multiplier $\beta$ is a function of $\vec{R}$, along the exterior surface $s$, as well as $\hat{\Omega}$ and $E$, and the constraints for the boundary conditions form an integral over angle, energy and the exterior surface s.

With this construction, the functions $\phi$ in the domain of $F$ now include discontinuous functions not satisfying the boundary conditions to be imposed on the neutron angular flux $\Psi$, since by requiring F to be stationary with respect to arbitrary variations in $\alpha$ and $\beta$, the conditions of Equations A.17 and A.18 appear as a consequence of the stationarity of the functional. Thus, if

$$
\delta_{\phi^{*}, \alpha, \beta}=0
$$


then $\phi$ must satisfy the equations:

$$
\begin{gathered}
{\left[\hat{\Omega} \cdot \nabla+\Sigma_{t}(\vec{r}, E)\right] \phi(r, \hat{\Omega}, E)=S(\vec{r}, \hat{\Omega}, E)} \\
+\iint_{4 \pi} d \hat{\Omega}^{\prime} \int_{0}^{\infty} d E^{\prime} \Sigma_{s}\left(\vec{r}, \hat{\Omega}, \hat{\Omega}^{\prime}, E, E^{\prime}\right) \phi\left(\vec{r}, \hat{\Omega}^{\prime}, E^{\prime}\right) \\
\phi\left(\vec{r}_{\sigma_{1}}, \hat{\Omega}, E\right)=\phi\left(\vec{r}_{\sigma_{2}}, \hat{\Omega}, E\right) \\
\phi(\vec{R}, \hat{\Omega}, E)=0, \hat{\Omega} \cdot \hat{s}<0
\end{gathered}
$$

The stationary conditions on the Lagrange multipliers $\alpha$ and $\beta$, as well as for $\phi^{*}$, are found by requiring the functional to be stationary with respect to arbitrary variation in the function $\phi$. Utilization of the identity

$$
\nabla \cdot\left(\hat{\Omega} \phi \phi^{*}\right)=\phi * \hat{\Omega} \cdot \nabla \phi+\phi \hat{\Omega} \cdot \nabla \phi^{*}
$$

allows the contribution to the functional from the gradient term to be reexpressed as

$$
\xi_{\mathrm{GRAD}}\left[\phi, \phi^{*}\right]=\left(\iiint_{\hat{R}_{I}} \overrightarrow{\mathrm{d}} \overrightarrow{\mathrm{r}}+\iiint_{\mathfrak{R}_{2}} \overrightarrow{\mathrm{d}} \overrightarrow{\mathrm{r}}\right) \iint_{4 \pi} \mathrm{d} \hat{\Omega} \int_{0}^{\infty} \mathrm{dE}\left\{\phi \hat{\Omega} \cdot \nabla \phi^{*}-\nabla\left(\hat{\Omega} \phi \phi^{*}\right)\right\}
$$

Now, application of the divergence theorem to the second term in Equation A.25 gives 


$$
\begin{gathered}
\mathscr{F}_{\mathrm{GRAD}}\left[\phi, \phi^{*}\right]=\iint_{4 \pi} \mathrm{d} \hat{\Omega} \int_{0}^{\infty} \mathrm{dE}\left\{\left(\iiint_{\Re_{1}} \mathrm{~d} \vec{r}+\iiint_{\widehat{R}_{2}} \mathrm{~d} \vec{r}\right) \phi \hat{\Omega} \cdot \nabla \phi^{*}\right. \\
+\iint_{\mathrm{s}} \mathrm{ds} \hat{\Omega} \cdot \hat{\mathrm{s}} \phi(\overrightarrow{\mathrm{R}}, \hat{\Omega}, \mathrm{E}) \phi^{*}(\overrightarrow{\mathrm{R}}, \hat{\Omega}, \mathrm{E}) \\
\left.+\iint_{\sigma} \mathrm{d} \sigma \hat{\Omega} \cdot\left[\hat{\sigma}_{1} \phi\left(\vec{r}_{\sigma_{1}}, \hat{\Omega}, \mathrm{E}\right) \phi^{*}\left(\vec{r}_{\sigma_{1}}, \hat{\Omega}, \mathrm{E}\right)+\hat{\sigma}_{2} \phi\left(\overrightarrow{\mathrm{r}}_{\sigma_{2}}, \hat{\Omega}, \mathrm{E}\right) \phi^{*}\left(\vec{r}_{\sigma_{2}}, \hat{\Omega}, \mathrm{E}\right)\right]\right\}
\end{gathered}
$$

The contribution to the functional from the scattering term may also be reexpressed by reversing the order of the primed and unprimed angular and energy integrations:

$$
\begin{aligned}
& \mathcal{F}_{\operatorname{SCAT}}\left[\phi, \phi^{*}\right]=\left(\iiint_{\mathfrak{R}_{1}} \mathrm{~d} \vec{r}+\iiint_{\Re_{2}} \mathrm{~d} \vec{r}\right) \iint_{4 \pi} \mathrm{d} \hat{\Omega}^{\prime} \int_{0}^{\infty} \mathrm{d} E^{\prime} \iint_{4 \pi} \mathrm{d} \hat{\Omega} \int_{0}^{\infty} \mathrm{dE}\left[\phi\left(\vec{r}, \hat{\Omega}^{\prime}, \vec{E}^{\prime}\right)\right. \\
& \left.\times \Sigma_{s}\left(\vec{r}, \hat{\Omega}, \hat{\Omega}^{\prime}, E, E^{\prime}\right) \phi^{*}(\vec{r}, \hat{\Omega}, E)\right]
\end{aligned}
$$

The primed and unprimed integration variables may now be interchanged to give

$$
\begin{aligned}
& { }^{F} \operatorname{SCAT}^{\left[\phi, \phi^{*}\right]}=\left(\iiint_{\mathcal{R}_{1}} \mathrm{~d} \vec{r}+\iiint_{R_{2}} \mathrm{~d} \vec{r}\right) \iint_{4 \pi} \mathrm{d} \hat{\Omega} \int_{0}^{\infty} \mathrm{dE} \iint_{4 \pi} \mathrm{d} \hat{\Omega}^{\prime} \int_{0}^{\infty} \mathrm{d} E^{\prime}[\phi(\vec{r}, \hat{\Omega}, \Xi) \\
& \left.\times \Sigma_{\mathrm{s}}\left(\vec{r}, \hat{\Omega}^{\prime}, \hat{\Omega}, \mathrm{E}^{\prime}, \mathrm{E}\right) \phi^{*}\left(\vec{r}, \hat{\Omega}^{\prime}, \mathrm{E}^{\prime}\right)\right]
\end{aligned}
$$

Insertion of the new expressions for the scattering and gradient terms in the functional of Equation A.19 then yields the alternate form of the functional; 


$$
\begin{aligned}
& \Re\left[\phi, \phi^{*}, \alpha, \beta\right]=\left(\iiint_{\mathbb{R}_{1}} d \vec{r}+\iiint_{\mathbb{R}_{2}} d \vec{r}\right) \iint_{4 \pi} d \hat{\Omega} \int_{0}^{\infty} d E\left\{\phi \left[S^{*}\right.\right. \\
& \left.\left.+\iint_{4 \pi} \mathrm{d} \hat{\Omega}^{\prime} \int_{0}^{\infty} \mathrm{d} E^{\prime} \Sigma_{\mathrm{S}}\left(\vec{r}, \hat{\Omega}^{\prime}, \hat{\Omega}, E^{\prime}, E\right) \phi^{*}\left(\vec{r}, \hat{\Omega}^{\prime}, E^{\prime}\right)+\hat{\Omega} \cdot \nabla \phi^{*}-\Sigma_{t^{*}} \phi^{*}\right]+S \phi^{*}\right\} \\
& +\iint_{\sigma} \mathrm{d} \sigma \iint_{4 \pi} \mathrm{d} \hat{\Omega} \int_{0}^{\infty} \mathrm{dE}\left\{\left(\hat{\sigma}_{1} \cdot \hat{\Omega}\right) \phi\left(\vec{r}_{\sigma_{1}}, \hat{\Omega}, \mathrm{E}\right)\left[\alpha\left(\vec{r}_{\sigma}, \hat{\Omega}, \mathrm{E}\right)-\phi^{*}\left(\vec{r}_{\sigma_{1}}, \hat{\Omega}, \mathrm{E}\right)\right]\right. \\
& +\left(\hat{\sigma}_{2} \cdot \hat{\Omega}\right) \phi\left(\vec{r}_{\sigma_{2}}, \hat{\Omega}, E\right)\left[\alpha\left(\vec{r}_{\sigma}, \hat{\Omega},(E)-\phi^{*}\left(\vec{r}_{\sigma_{2}}, \hat{\Omega}, E\right)\right]\right\} \\
& +\iint_{S} d s \iint_{4 \pi} d \hat{\Omega} \int_{0}^{\infty} d E\left\{H(-\hat{\Omega} \cdot \hat{s})(\hat{\Omega} \cdot \hat{s}) \phi(\vec{R}, \hat{\Omega}, E)\left[B(\vec{R}, \hat{\Omega}, E)-\phi^{*}(\vec{R}, \hat{\Omega}, E)\right]\right. \\
& \left.+H(\hat{\Omega} \cdot \hat{\mathrm{s}})(\hat{\Omega} \cdot \hat{\mathrm{s}}) \phi(\overrightarrow{\mathrm{R}}, \hat{\Omega}, \mathrm{E})\left[\phi^{*}(\overrightarrow{\mathrm{F}}, \hat{\Omega}, \mathrm{E})\right]\right\}
\end{aligned}
$$

Now, requiring that

$$
\delta F_{\phi}=0
$$

requires that each of the square bracketed terms in Equation A.29 vanish identically. Thus, at the stationary point, $\phi^{*}, \alpha$ and $\beta$ must satisfy the conditions

$$
\begin{gathered}
{\left[-\hat{\Omega} \cdot \nabla+\Sigma_{t}(\vec{r}, E)\right] \phi^{*}(\vec{r}, \hat{\Omega}, E)=S^{*}(\vec{r}, \hat{\Omega}, E)} \\
+\iint_{4 \pi} d \hat{\Omega}^{\prime} \int_{0}^{\infty} d E^{\prime} \Sigma_{s}\left(\vec{r}, \hat{\Omega}^{\prime}, \hat{\Omega}, E^{\prime}, E\right) \phi^{*}\left(\vec{r}, \hat{\Omega}^{\prime}, E^{\prime}\right) \\
\phi^{*}\left(\vec{r}_{\sigma_{1}}, \hat{\Omega}, E\right)=\alpha\left(\vec{r}_{\sigma}, \hat{\Omega}, E\right)=\phi^{*}\left(\vec{r}_{\sigma_{2}}, \hat{\Omega}, E\right) \\
\phi^{*}(\vec{R}, \hat{\Omega}, E)=0, \hat{\Omega} \cdot \hat{s}>0
\end{gathered}
$$




$$
\beta(\vec{R}, \hat{\Omega}, E)=\phi^{*}(\vec{R}, \hat{\Omega}, E), \hat{\Omega} \cdot \hat{s}<0
$$

Equations A.31 through A.33 are just the adjoint transport equations and appropriate boundary and continuity conditions. Thus, at the stationary point, $\phi^{*}$ must be the adjoint neutron angular flux. Further, $\alpha\left(\vec{r}_{\sigma}, \hat{\Omega}, E\right)$ is seen to be the stationary value of the adjoint angular flux along the interior surface $\sigma$, and $\beta(\vec{R}, \hat{\Omega}, E)$ is seen to be the stationary value of the incoming adjoint angular flux along the exterior surface $s$.

If, as in the classical applications of constraint conditions by Lagrange multipliers, the exact solution for the multipliers are obtained, Equations A.32 and A.34 imply that the solution to the adjoint problem must be obtained since the multipliers are given in terms of the adjoint function $\phi^{*}$. Avoiding an adjoint solution, the Lagrange multipliers must themselves be approximated, and as a consequence, the approximate solutions obtained by the variational synthesis method will not satisfy the boundary and continuity conditions exactly, but only in a weighted integral sense. The Lagrange multipliers might therefore be assigned the dependence on the functions $\phi^{*}$ as indicated in Equations A.32 and A.34. For the multiplier $\beta$, no difficulty in this assignment arises, but for the multiplier $\alpha$, a problem arises because, with the admission of discontinuous functions $\phi^{*}$, it is possible that

$$
\phi *\left(\vec{r}_{\sigma_{1}}, \hat{\Omega}, E\right) \neq \phi *\left(\vec{r}_{\sigma_{2}}, \hat{\Omega}, E\right)
$$

This problem is overcome by assigning $\alpha$ the value 


$$
\alpha\left(\overrightarrow{r_{\sigma}}, \hat{\Omega}, E\right)=H\left(\hat{\Omega} \cdot \hat{\sigma}_{1}\right) \phi^{*}\left(\vec{r}_{\sigma_{2}}, \hat{\Omega}, E\right)+H\left(\hat{\Omega} \cdot \hat{\sigma}_{2}\right) \phi^{*}\left(\vec{r}_{\sigma_{1}}, \hat{\Omega}, E\right)
$$

as proposed by $0^{\prime}$ Reilly ${ }^{26}$ and used by Lancefield. ${ }^{30}$ With this assignment, the stationary condition on $\phi^{*}$ glven by A.32 is replaced by the two equivalent conditions

$$
\begin{aligned}
& H\left(\hat{\Omega} \cdot \hat{\sigma}_{1}\right) \phi^{*}\left(r_{\sigma_{2}}, \hat{\Omega}, E\right)=H\left(-\hat{\Omega}_{0} \cdot \hat{\sigma}_{2}\right) \phi^{*}\left(r_{\sigma_{2}}, \hat{\Omega}, E\right) \\
& H\left(\hat{\Omega} \cdot \hat{\sigma}_{2}\right) \phi^{*}\left(r_{\sigma_{2}}, \hat{\Omega}, E\right)=H\left(-\hat{\Omega} \cdot \hat{\sigma}_{1}\right) \phi^{*}\left(r_{\sigma_{1}}, \hat{\Omega}, E\right)
\end{aligned}
$$

Similarly, the continuity conditions on the stationary function $\phi$ as a result of Equation A.36 become

$$
\mathrm{H}\left(\hat{\Omega} \cdot \hat{\sigma}_{1}\right) \phi\left(\vec{r}_{\sigma_{1}}, \hat{\Omega}, E\right)=H\left(-\hat{\Omega} \cdot \hat{\sigma}_{2}\right) \phi\left(\vec{r}_{\sigma_{2}}, \hat{\Omega}, E\right)
$$

and

$$
\mathrm{H}\left(\hat{\Omega} \cdot \hat{\sigma}_{2}\right) \phi\left(\vec{r}_{\sigma_{2}}, \hat{\Omega}, E\right)=H\left(-\hat{\Omega} \cdot \hat{\sigma}_{1}\right) \phi\left(\vec{r}_{\sigma_{1}}, \hat{\Omega}, E\right)
$$

Equation A.39 implies that neutrons leaving $\Re_{1}$ through the surface $\sigma$ must enter $R_{2}$, and Equation A.40 implies that neutrons leaving $R_{2}$ through $\sigma$ must enter $\mathfrak{R}_{1}$. These two conditions are, of course, physically equivalent to the original continuity condition A.18. Having expressions for the Lagrange multipliers $\alpha$ and $\beta$, the functional of Equation A.19 can be simplified by substitution of Equations A.36 and A.34 for the values of $\alpha\left(\vec{r}_{\sigma}, \hat{\Omega}, \vec{E}\right)$ and $\beta(\vec{R}, \hat{\Omega}, E)$. The final form of the functional for the two-region case thus becomes: 


$$
\begin{aligned}
& \mathscr{F}\left[\phi, \phi^{*}\right]=\left(\iiint_{\mathscr{R} 1} \mathrm{~d} \overrightarrow{\mathbf{r}}+\iiint_{\mathscr{R} Z} \mathrm{~d} \overrightarrow{\mathbf{r}}\right) \iint_{4 \pi} \mathrm{d} \hat{\Omega} \int_{0}^{\infty} \mathrm{dE}\left\{\phi^{*}[\mathrm{~S}\right. \\
& \left.\left.+\iint_{4 \pi} \mathrm{d} \hat{\Omega}^{\prime} \int_{0}^{\infty} \mathrm{d} E^{\prime} \Sigma_{s}\left(\vec{r}, \hat{\Omega}, \hat{\Omega^{\prime}}, E, E^{\prime}\right) \phi\left(\vec{r}, \hat{\Omega}^{\prime}, E^{\prime}\right)-\hat{\Omega} \cdot \nabla \phi-\Sigma_{t} \phi\right]+S^{*} \phi\right\} \\
& +\iint_{\sigma} d \sigma \int_{4 \pi} d \hat{\Omega} \int_{0}^{\infty} d E\left\{\left[H\left(-\hat{\Omega} \cdot \hat{\sigma}_{1}\right) \phi *\left(\vec{r}_{\sigma_{1}}, \hat{\Omega}, E\right)+\mathbf{H}\left(-\hat{\Omega}^{*} \cdot \hat{\sigma}_{2}\right) \phi *\left(\vec{r}_{\sigma_{2}}, \hat{\Omega}, \vec{\Xi}\right)\right]\right. \\
& \left.\times\left(\hat{\Omega} \cdot \hat{\sigma}_{1}\right)\left[\phi\left(\vec{r}_{\sigma_{1}}, \hat{\Omega}, E\right)-\phi\left(\vec{r}_{\sigma_{2}}, \hat{\Omega}, \mathrm{E}\right)\right]\right\} \\
& +\iint_{\mathrm{S}} \mathrm{ds} \iint_{4 \pi} \mathrm{d} \hat{\Omega} \int_{0}^{\infty} \mathrm{dE}\left\{\mathrm{H}(\hat{\Omega} \cdot \hat{\mathrm{s}}) \phi^{*}(\overrightarrow{\mathrm{R}}, \hat{\Omega}, E)(\hat{\Omega} \cdot \hat{\mathrm{s}}) \phi(\overrightarrow{\mathrm{R}}, \hat{\Omega}, E)\right\}
\end{aligned}
$$

The Multiregion Reactor

Equation A.4l may be generalized to the case of a multiregion reactor, divided arbitrarily into $\Lambda$ subregions by the interior surfaces $\sigma_{\lambda}$, with the help of the following notation:

$R_{\lambda}$ denotes the $\lambda$ th subregion $(\lambda=1,2, \ldots \Lambda)$,

$s_{\lambda}$ is that portion of the exterior surface $s$ belonging to $R_{\lambda}$,

$\{\nu(\lambda)\}$ is the set of indices of subregions contiguous with $R_{\lambda}$,

$\sigma_{\lambda, \nu(\lambda)}$ denotes the common interior surface between $R_{\lambda}$ and $R_{\nu(\lambda)}$,

$\hat{s}_{\lambda}$ is outward unit normal to $s_{\lambda}$,

$\hat{\sigma}_{\lambda}$ is the outward unit normal to surface of $R_{\lambda}$,

$\vec{r}_{\lambda, \nu}(\lambda)$ is a point on $\sigma_{\lambda, \nu}(\lambda)$ approached from within $R_{\lambda}$,

$\vec{r}_{\nu(\lambda), \lambda}$ represents the same point approached from within $R_{\nu}(\lambda)$.

With these definitions, Equation A.4l generalizes to: 


$$
\begin{aligned}
& F\left[\phi, \phi^{*}\right]=\sum_{\lambda=\mathcal{I}}^{\Lambda}\left(\iiint_{\lambda} \mathrm{d} \overrightarrow{\mathrm{r}} \iint_{4 \pi} \mathrm{d} \hat{\Omega} \int_{0}^{\infty} \mathrm{dE}\{\phi *(\overrightarrow{\mathrm{r}}, \hat{\Omega}, \mathrm{E})[\mathrm{s}(\overrightarrow{\mathrm{r}}, \hat{\Omega}, E)\right. \\
& +\iint_{4 \pi} d \hat{\Omega}^{\prime} \int_{0}^{\infty} d E^{\prime} \Sigma_{s}\left(\vec{r}, \hat{\Omega}, \hat{\Omega}^{\prime}, E, \mathbb{F}^{\prime}\right) \phi\left(\vec{r}, \hat{\Omega}^{\prime}, E^{\prime}\right) \\
& \left.\left.-\hat{\Omega} \cdot \nabla \phi(\vec{r}, \hat{\Omega}, E)-\Sigma_{t}(\vec{r}, E) \phi(\vec{r}, \hat{\Omega}, E)\right]+s^{*}(\vec{r}, \hat{\Omega}, E) \phi(\vec{r}, \hat{\Omega}, E)\right\} \\
& +\sum_{\nu(\lambda)} \iint_{\sigma_{\lambda, \nu(\lambda)} \mathrm{d} \sigma} \iint_{4 \pi} \mathrm{d} \hat{\Omega} \int_{0}^{\infty} \mathrm{dE}\left\{\mathrm{H}\left(-\hat{\Omega} \cdot \hat{\sigma}_{\lambda}\right)\left(\hat{\Omega} \cdot \hat{\sigma}_{\lambda}\right) \phi^{*}\left(\overrightarrow{\mathbf{r}}_{\lambda, \nu(\lambda)}, \hat{\Omega}, E\right)\right. \\
& \left.\times\left[\phi\left(\overrightarrow{\mathrm{r}}_{\lambda, \nu(\lambda)}, \hat{\Omega}, \mathrm{E}\right)-\phi\left(\overrightarrow{\mathrm{r}}_{\nu(\lambda), \lambda}, \hat{\Omega}, \mathrm{E}\right)\right]\right\} \\
& \left.+\iint_{s_{\lambda}} d s \iint_{4 \pi} d \hat{\Omega} \int_{0}^{\infty} d E\left\{H\left(-\hat{\Omega} \cdot \hat{s}_{\lambda}\right) \phi *(\vec{R}, \hat{\Omega}, E)\left(\hat{\Omega} \cdot \hat{s}_{\lambda}\right) \phi(\vec{R}, \hat{\Omega}, E)\right\}\right)
\end{aligned}
$$

Since the division into subregions was arbitrarily made, this form of the functional admits within its domain, functions $\phi$ and $\phi^{*}$ which are discontinuous along any interior surfaces, and has, as its stationary functions, the continuous solutions to the forward and adjoint transport equation. It may thus be used to derive approximations of $\Psi$ and \%* in terms of discontinuous trial functions $\phi$ and $\phi^{*}$. 
APPENDIX B

REDUCED EQUATIONS FOR THE THREE-DIMENSIONAL CASE

Utilization of the variational symthesis method to obtain approximate solutions to the three-dimensional rectangular reactor, as described in Chapter III, is accomplished by choosing forward and adjoint trial functions of the form of Equation 3.53 to remove the base function variables, and treating the angular dependence in a discrete ordinates approximation using a trial function as in Equations 3.42 and 3.43. Heving reduced the multigroup functional by this two-step procedure, the equation for the discrete angular mixing functions $\phi_{\text {gnmd }}(z)$ are found, by requiring the reduced functional to be stationary with respect to arbitrary variations in the $\phi_{\text {gnmd }}^{*}(z)$, to be

$$
\begin{aligned}
& \xi_{\mathrm{d}} \mathrm{A}_{\text {gnm } \ell}^{\mathrm{I}} \frac{\mathrm{d}}{\mathrm{dz}} \phi_{\text {gnmd }}(\mathrm{z})+\left[\Sigma_{\text {tgnm } \ell}^{\mathrm{I}}+\mu_{\mathrm{d}} \mathrm{B}_{\text {gnm } \ell}^{\mathrm{I}}+r_{\mathrm{d}} \mathrm{E}_{\text {gnm } \ell}^{\mathrm{I}}\right] \phi_{\text {gnmd }}(\mathrm{z})=\mathrm{s}_{\text {gnm } \ell \mathrm{d}}^{\mathrm{I}} \\
& +\mu_{d} C_{g n m \ell}^{I}\left\{\left(1-\delta_{n_{1}}\right)\left[\phi_{\text {gn-Imd }}(z)-\phi_{\text {gnmd }}(z)\right]+\delta_{n 1} \phi_{\text {gnmd }}(z)\right\} \\
& +\eta_{\mathrm{d}} \mathrm{F}_{\mathrm{gnm} \ell}^{\mathrm{I}}\left\{\left(1-\delta_{\mathrm{ml}}\right)\left[\phi_{\mathrm{gnm}-1 \mathrm{~d}}(z)-\phi_{\mathrm{gnmd}}(z)\right]\right. \\
& \left.+\delta_{m 1} \phi_{\text {gnmd }}(z)\right\} ; d=1,2, \ldots, \frac{D}{4}
\end{aligned}
$$


141

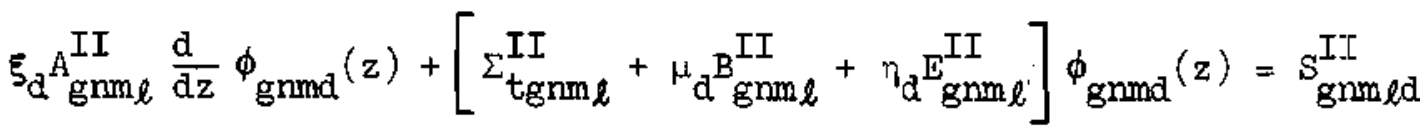

$$
\begin{aligned}
& +\mu_{d} c_{g n+1 m \ell}^{I I}\left\{\left(1-\delta_{n N}\right)\left[\phi_{g n m d}(z)-\phi_{g n+1 m d}(z)\right]+\delta_{n N} \phi_{g n m d}(z)\right\} \\
& +\eta_{\mathrm{d}} \mathrm{F}_{\text {gnm } \ell}^{\mathrm{II}}\left\{\left(1-\delta_{\mathrm{m} 1}\right)\left[\phi_{\mathrm{gnm}-1 \mathrm{~d}}(z)-\phi_{\text {gnmd }}(z)\right]\right. \\
& \left.+\delta_{m 1} \phi_{g n m d}(z)\right\} ; d=\frac{D}{4}+1, \ldots, \frac{D}{2}
\end{aligned}
$$

$$
\begin{aligned}
& \xi_{\mathrm{d}} \mathrm{A}_{\text {gnm } \ell}^{\text {III }} \frac{\mathrm{d}}{\mathrm{dz}} \phi_{\text {gnmd }}(z)+\left[\Sigma_{\text {tgnm } \ell}^{\text {III }}+\mu_{\mathrm{d}} \mathrm{B}_{\text {gnm } \ell}^{\text {III }}+\eta_{\mathrm{d}} E_{\text {gnm } \ell}^{\text {III }}\right]_{\text {gnmd }}(z)=s_{\text {gnm } \ell d}^{\text {III }} \\
& +\mu_{d} C_{g n+I m \ell}^{I I I}\left\{\left(1-\delta_{n N}\right)\left[\phi_{g n m d}(z)-\phi_{g n+j m d}(z)\right]+\delta_{n N} \phi_{g n m d}(z)\right\} \\
& +\eta_{d} F_{g n m+2 \ell}^{I I I}\left\{\left(1-\delta_{m M}\right)\left[\phi_{\text {gnmd }}(z)-\phi_{g n m+1 d}(z)\right]\right. \\
& \left.+\delta_{n M_{\text {gnmd }}}(z)\right\} ; d=\frac{D}{2}+3, \ldots, \frac{3 D}{4}
\end{aligned}
$$

$$
\begin{aligned}
& \xi_{\mathrm{d}} \mathrm{A}_{\text {gnm } \ell}^{\mathrm{IV}} \frac{\mathrm{d}}{\mathrm{dz}} \phi_{\text {gnmd }}(z)+\left[\Sigma_{\text {tgnm } \ell}^{\mathrm{IV}}+\mu_{\mathrm{d}} \mathrm{B}_{\text {gnm } \ell}^{\mathrm{IV}}+r_{\mathrm{d}} \mathrm{E}_{\text {gnm } \ell}^{\mathrm{IV}}\right] \phi_{\text {gnmd }}(z)=s_{\text {gnm } \ell \mathrm{d}}^{\mathrm{IV}} \\
& +\mu_{d} C_{\text {gnm } \ell}^{I V}\left\{\left(1-\delta_{n I}\right)\left[\phi_{g n-I m d}(z)-\phi_{\text {gnmd }}(z)\right]+\delta_{n_{I} \phi_{\text {gnmd }}}(z)\right\} \\
& +\eta_{d} F_{g n m+1 \ell}^{I V}\left\{\left(1-\delta_{m M}\right)\left[\phi_{g n m d}(z)-\phi_{g n m+1 d}(z)\right]\right. \\
& \left.+\delta_{\mathrm{mM}} \phi_{\text {gnmd }}(z)\right\} ; d=\frac{3 D}{4}+1, \ldots, D
\end{aligned}
$$

$$
\mathrm{D}_{\text {gnm } \ell \ell-1}^{\alpha} \phi_{\text {gnmd }}\left(z_{\ell-1, \ell}\right)=A_{\text {gnm } \ell}^{\alpha} \phi_{\text {gnnd }}\left(z_{\ell, \ell-1}\right) ; \xi_{d}>0, \alpha=I, \ldots, I V
$$

$$
A_{\text {gnm } \ell}^{\alpha} \phi_{\text {gnmd }}\left(z_{\ell, \ell+1}\right)=D_{\text {gnm } \ell \ell+1}^{\alpha} \phi_{\text {gnmd }}\left(z_{\ell+1, \ell}\right) ; \xi_{d}<0, \alpha=I, \ldots, I V
$$




$$
\begin{gathered}
\phi_{\text {gnnd }}\left(z_{1}\right)=0 ; \xi_{d}>0 \\
\phi_{\text {gnmd }}\left(z_{\text {I }+1}\right)=0 ; \xi_{d}<0
\end{gathered}
$$

In these equations, the meaning of the superscripts and the placenent of the discrete segments by quadrant are as described in Chapter III. Also the value of the superscript $\alpha$, where $\alpha=I$, II, III, IV, in Equations B.le and B.If are determined from the value of the subscript $d$ as specified in Chapter III. $\xi_{d}$ is the discrete direction cosine along the $z$ axis, and is defined analogous to the discrete $x$ and $y$ cosines, $\mu_{d}$ and $\eta_{\tilde{d}}$, as given in Equations 3.48 and 3.49 . The consistent definitions of the matrix synthesis constants appearing in these equations, analogous to the definitions for the two-dimensional case, are

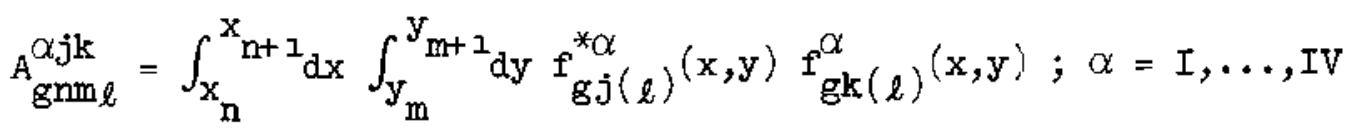

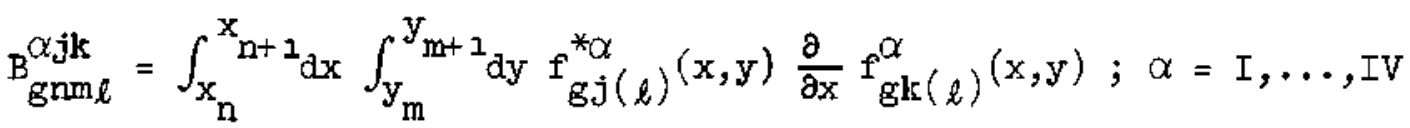

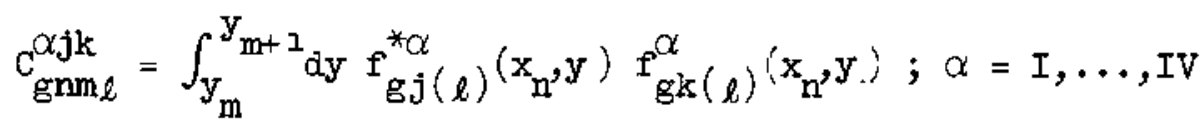

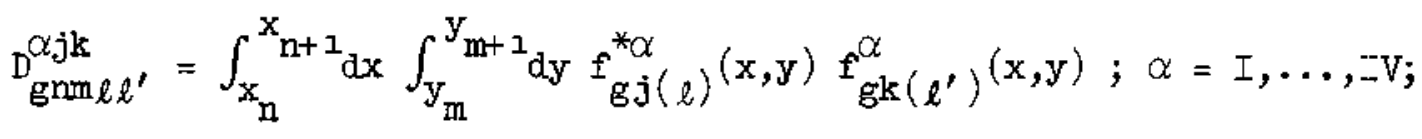

$$
\begin{aligned}
& \ell^{\prime}=\ell^{+1, \ell-1} \quad(\mathrm{~B} .5)
\end{aligned}
$$




$$
\begin{aligned}
& \mathrm{E}_{\mathrm{gnml}}^{\alpha j k}=\int_{x_{n}}^{x_{n+1}} \mathrm{dx} \int_{y_{m}}^{y_{m+1}} d y f_{g j(\ell)}^{* \alpha}(x, y) \frac{\partial}{\partial y} f_{g k(\ell)}^{\alpha}(x, y) ; \alpha=I, \ldots, I V \\
& \mathrm{~F}_{\mathrm{gnm \ell}}^{\alpha j \mathrm{k}}=\int_{\mathrm{x}_{\mathrm{n}}}^{\mathrm{x}_{\mathrm{n}+1} \mathrm{dx}} \mathrm{f}_{\mathrm{gj}(\ell)}^{* \alpha}\left(\mathrm{x}, \mathrm{y}_{\mathrm{m}}\right) \mathrm{f}_{\mathrm{gk}(\ell)}^{\alpha}\left(\mathrm{x}, \mathrm{y}_{\mathrm{m}}\right) ; \alpha=\mathrm{I}, \ldots, \mathrm{IV} \\
& \Sigma_{\text {tgnm } \ell}^{\alpha j k}=\int_{x_{n}}^{x_{n+1}} d x \int_{y_{m}}^{y_{m+1}} d y f_{g j(\ell)}^{* \alpha}(x, y) \Sigma_{t g \ell}(x, y) f_{g k(\ell)}^{\alpha}(x, y) ; \\
& \alpha=I, \ldots, I V
\end{aligned}
$$

These equations for the three-dimensional case are seen to be formally similar to those of the two-dimensional case. In this case, however, there are four equations specifying the dependence of the discrete angular mixing functions along the remaining spatial variable: one for each of the four ranges of the angular segmentation. The same directional sense in the channel coupling for these equations is observed, as was observed for the two-dimensional equations. Now however, each channel is coupled to the four adjacent channels, two along each of the base function axes $x$ and $y$. Thus, for example, channel $n, m$ is coupled to channel $n, m+l$ through neutrons with $\eta_{\mathrm{d}}<0$, to channel $\mathrm{n}, \mathrm{m}-1$ through neutrons with $\eta_{\mathrm{d}}>0$, to channel $\mathrm{n}+1, \mathrm{~m}$ through neutrons with $\mu_{\mathrm{d}}<0$, and to channel $\mathrm{n}-1, \mathrm{~m}$ through neutrons with $\mu_{\mathrm{d}}>0$. I.hese simflarities are also evident in the boundary and continuity conditions of Equations B.le through B.Ih.

Equations B.I may be solved, as in the two-dimensional case, by defining a fine mesh, $I=1,2, \ldots, I M A X$, along the remaining spatial 
variable, sweeping this mesh by channel in a directional sense to determine each iterate solution and utilizing the iteration procedure as described in Chapter III. Having converged the iterative procedure, the approximate solution is then determined by recombining the resultIng discrete angular mixing functions with the base functions by use of the trial function expression. 


\section{APPENDIK C}

\section{REFLECTED BOUNDARY CONDITIONS}

For many applications, the boundary conditions to be imposed on the Boltzmann are of the reflected type, i.e., all neutrons encountering the outer surface are returned to the system by mirror reflection. Such instances often arise, for example, in reactor cell calculations. These conditions can be treated consistently in variational synthesis method by using an appropriate term in the functional and will be develojped here for the case of a two-dimensional rectangular reactor (Figure 1 ) and a single energy group. Writing the two components of the angular variable explicity, the reflected boundary conditions for this case may be written

$$
\Psi(x, y, \mu, \eta)=\Psi(x, y,-\mu, \eta) ; x=x_{3}, x_{N+1}
$$

and

$$
\Psi(x, y, \mu, \eta)=\Psi(x, y, \mu,-\eta) ; y=y_{1}, y_{M+1}
$$

An appropriate term for replacing the vacuum boundary condition term in the functional is 


$$
\begin{aligned}
& F_{B . C}\left[\phi, \phi^{*}\right]=\sum_{m=1}^{M} \iint_{4 \pi} d \hat{\Omega} \int_{y_{m}}^{y_{m+1}} d y\left\{H ( - \Omega _ { x } ) \phi ^ { * } ( x _ { N + 1 } , y , \mu , \eta ) \Omega _ { x } \left[\phi\left(x_{N+1}, y, \mu, \eta\right)\right.\right. \\
& \left.\left.-\phi\left(\mathrm{x}_{\mathrm{N}+1}, \mathrm{y},-\mu, \eta\right)\right]+\mathrm{H}\left(\Omega_{\mathrm{x}}\right) \phi^{*}\left(\mathrm{x}_{1}, \mathrm{y}, \mu, \eta\right) \Omega_{\mathrm{x}}\left[\phi\left(\mathrm{x}_{1}, \mathrm{y},-\mu, \eta\right)-\phi\left(\mathrm{x}_{1}, \mathrm{y}, \mu, \eta\right)\right]\right\} \\
& +\sum_{n=1}^{\mathbb{N}} \iint_{4 \pi} d \hat{\Omega} \int_{x_{n}}^{x_{n+1}}\left\{H ( - \Omega _ { y } ) \phi ^ { * } ( x , y _ { M + 1 } , \mu , \eta ) \Omega _ { y } \left[\phi\left(x, y_{M+1}, \mu, \eta\right)\right.\right. \\
& \left.\left.-\phi\left(x, y_{M_{1}}, \mu,-\eta\right)\right]+H\left(\Omega_{y}\right) \phi^{*}\left(x, y_{1}, \mu, \eta\right) \Omega_{y}\left[\phi\left(x, y_{1}, \mu,-\eta\right)-\phi\left(x, y_{1}, \mu, \eta\right)\right]\right\}
\end{aligned}
$$

The vanishing of the functional $\mathscr{F}$, thus appended, with respect to arbitrary variations in the functions $\phi^{*}(\mathrm{x}, \mathrm{y}, \mu, \eta)$ requtres that each of the square bracketed terms in Equation C.3 vanish identically. Hence the stationary functions $\Psi$ satisfy Equations $C .1$ and $C .2$ and this term is an appropriate variational characterization of the reflected boundary conditions.

Inserting the trial function expressjons of Equations 3.29 and 3.30, performing the integration over the base function $x$, the reduced term becomes

$$
\begin{aligned}
& F_{B . C .}\left[\phi, \phi^{*}\right]=\sum_{m=1}^{M} \iint_{4 \pi} d \hat{\Omega} \int_{y_{m}}^{y_{m+1}} \int_{H}\left(-\Omega_{x}\right) \phi_{N}^{*-}(y, \mu, \eta) \Omega_{x}\left[C_{N+1, m_{N}^{-}}^{-}\left(y, \mu, \eta_{1}\right)\right. \\
& \left.-\mathrm{U}_{\mathrm{m}} \phi_{\mathrm{N}}^{+}(\mathrm{y},-\mu, \eta)\right]+\mathrm{H}\left(\Omega_{\mathrm{x}}\right) \phi_{1}^{*+}(\mathrm{y}, \mu, \eta) \Omega_{\mathrm{x}}\left[\mathrm{V}_{\mathrm{m}} \phi_{1}^{-}\left(\mathrm{y}, \neg^{\mu, \eta}\right)-\mathrm{C}_{\left.\mathrm{Im}^{+} \phi^{+}\left(\mathrm{y}, \mu, \tau_{\mathrm{T}}\right)\right]}^{\}}\right.
\end{aligned}
$$




$$
\begin{aligned}
& +\sum_{n=1}^{N} \iint_{\pi} d \hat{\Omega}\left\{H ( - \Omega _ { y } ) H ( - \Omega _ { x } ) \phi _ { n } ^ { * - } ( y _ { M + 1 } , \mu , \eta ) \Omega _ { y } A _ { n M } ^ { - } \left[\phi_{n}^{-}\left(y_{M+1}, \mu, \eta\right)\right.\right. \\
& \left.-\phi_{n}^{-}\left(y_{M+1}, \mu,-\eta\right)\right] \\
& +H\left(-\Omega_{y}\right) H\left(\Omega_{x}\right) \phi_{n}^{*+}\left(y_{M+1}, \mu, \eta\right) \Omega_{y} A_{n M}^{+}\left[\phi_{n}^{+}\left(y_{M+1}, \mu, \eta\right)-\phi_{n}^{+}\left(y_{M+1}, \mu,-\eta\right)\right] \\
& +H\left(\Omega_{y}\right) H\left(-\Omega_{x}\right) \phi_{n}^{*}-\left(y_{1}, \mu, \eta\right) \Omega_{y} A_{n 1}^{-}\left[\phi_{n}^{-}\left(y_{1}, \mu,-\eta\right)-\phi_{n}^{-}\left(y_{1}, \mu, \eta\right)\right] \\
& \left.+H\left(\Omega_{y}\right) H\left(\Omega_{x}\right) \phi_{n}^{*+}\left(y_{1}, \mu, \eta\right) \Omega_{y} A_{n 1}^{+}\left[\phi_{n}^{+}\left(y_{1}, \mu,-\eta\right)-\phi_{n}^{+}\left(y_{1}, \mu, \eta\right)\right]\right\}
\end{aligned}
$$

where the matrices $C^{ \pm}$and $A^{ \pm}$are as defined by Equations 3.35 and 3.33 and the mixing functions are expressed in matrix form. The matrices $\mathrm{U}_{\mathrm{m}}$ and $\mathrm{V}_{\mathrm{m}}$ are defined so that their $\mathrm{j}, \mathrm{kth}$ elements are given by

$$
\mathrm{U}_{\mathrm{m}}^{\mathrm{jk}}=\mathrm{f}_{\mathrm{j}(\mathrm{m})}^{*}\left(\mathrm{x}_{\mathrm{N}+\mathrm{I}}\right) \mathrm{f}_{\mathrm{k}(\mathrm{m})}^{+}\left(\mathrm{x}_{\mathrm{H}+1}\right)
$$

and

$$
\mathrm{V}_{m}^{\mathrm{jk}}=\mathrm{f}_{j(\mathrm{~m})}^{*+}\left(\mathrm{x}_{1}\right) \mathrm{f}_{\mathrm{k}(\mathrm{m})}^{-}\left(\mathrm{x}_{1}\right)
$$

In obtaining $\mathrm{C} .4$ and $\mathrm{C} .3$ by insertion of the trial function, use has been made of the fact that

$$
\iint_{4 \pi} d \hat{\Omega} H\left(\Omega_{x}\right) H\left(-\Omega_{x}\right)=0
$$

The discrete angular approximation is made using the trial functions for the angular dependent mixing functions as given in Equations 3.42 through 3.45. Consider now the first term in the first 
integral of Equation C.4. Inserting the trial function expressions, this term becomes

$$
\begin{gathered}
\sum_{m=1}^{M} \int_{y_{m}}^{y_{m+1}} d y \iint_{4 \pi} d \Omega H\left(-\Omega_{x}\right) \sum_{d=1}^{D / 2} \phi_{N d}^{*}(y) \Delta_{d}(\mu, \eta) \Omega_{x}\left[C_{N+1 m}^{-} \sum_{d^{\prime}=1}^{D / 2} \phi_{N^{\prime} d^{\prime}}(y) \Delta_{d}(\mu, \pi)\right. \\
\left.-U_{m} \sum_{d^{\prime}=\frac{D}{2}+1}^{D} \phi_{N d^{\prime}}(y) \Delta_{d^{\prime}}(-\mu, \eta)\right]
\end{gathered}
$$

where the angular dependence of the step function $\Delta_{d}$ has been written explicitly. The angular integration is now performed to obtain

$$
\sum_{m=1}^{M} \sum_{d=1}^{D / 2} \int_{y_{m}}^{y_{m+1}} \phi_{N d}^{*}(y) \mu_{d}\left[C_{N+1 m}^{-} \phi_{N d}(y)-U_{m} \phi d+\frac{D^{2}}{2}(y)\right]
$$

Note that the double sum over $d$ and $d^{\prime}$ has been reduced to a sing le sum, in the first instance, by the fact that

$$
\int \mathrm{d} \hat{\Omega} \Delta_{d^{\prime}} \Delta_{d^{\prime}} \equiv 0 ; d \neq d^{\prime}
$$

and in the second instance by the fact that

$$
\int \mathrm{d} \hat{\Omega} H\left(\Omega_{\mathrm{x}}\right) \Delta_{\mathrm{d}}(\mu, \eta) \Delta_{d^{\prime}}(-\mu, \eta) \equiv 0 ; a^{\prime} \neq \mathrm{d}+\frac{\mathrm{D}}{2}
$$

The second fact is true by virtue of the symmetry of the angular segmentation and the ordering of the segments such that the index of the discrete segment, $d$, having cosines $\mu_{d}, \eta_{d}\left(\mu_{d}>0\right)$ is related to the index of the discrete segment, $d^{\prime}$, having cosines $\neg_{d}$, $\eta_{d}$ by the relation 


$$
d^{\prime}=d-\frac{D}{2}
$$

Similar considerations for the other terms in Equation C. 4 yield the discrete form to be

$$
\begin{aligned}
& F_{\text {B.C. }}\left[\phi_{n d}, \phi_{n d}^{*}\right]=\sum_{m=1}^{M} \int_{y_{m}}^{y_{m+1} d y}\left\{\sum_{d=1}^{D / 2} \phi_{N d}^{*}(y) \mu_{d}\left[C_{N+1 m}^{-} \phi_{N d}(y)-U_{m} \phi_{N d}+\frac{D}{2}(y)\right]\right. \\
& +\sum_{d=\frac{D}{2}+1}^{D} \phi_{1 d}^{*}(y) \mu_{d}\left[V_{m} \phi\left(1 d-\frac{D}{2}(y)-C_{1 m}^{+} \phi 1 d(y)\right]\right\} \\
& +\sum_{n=1}^{N}\left\{\sum_{d=1}^{D / 4} \phi_{n d}^{*}\left(y_{1}\right) \eta_{d} A_{n M}^{-}\left[\phi{ }_{n d}+D_{\frac{D}{4}}^{\left(y_{1}\right)}-\phi_{n d}\left(y_{1}\right)\right]\right. \\
& +\sum_{d=\frac{D}{4}+1}^{D / 2} \phi_{n d}^{*}\left(y_{M+1}\right) \eta_{d} A_{n 1}^{-}\left[\phi_{n d}\left(y_{M+1}\right)-\phi_{n d}-\frac{D}{4}\left(y_{M+1}\right)\right] \\
& +\sum_{d=\frac{D}{2}+1}^{3 D / 4} \phi_{n d}^{*}\left(y_{1}\right) \eta_{d} A_{n 1}^{+}\left[\phi n d+\frac{D}{4}\left(y_{1}\right)-\phi_{n d}\left(y_{1}\right)\right] \\
& \left.+\sum_{d=\frac{3 D}{4}+1}^{D} \phi_{n d}^{*}\left(y_{M+1}\right) \eta_{d} A_{n M}^{+}\left[\phi_{n d}\left(y_{M+1}\right)-\phi_{n d-\frac{D}{4}}\left(y_{M+1}\right)\right]\right\}
\end{aligned}
$$

The requirement that the reduced functional in discrete form, with the boundary term as given by C.11, be stationary with respect to variations on the $\phi_{\text {nd }}^{*}$ then yields the equation and condition for the discrete angular mixing functions. The equations are the same as 
$3.46 \mathrm{a}$ and $3.46 \mathrm{~b}$ with the exception that the last term in each equation, $\mu_{d} c_{n+1, m}^{-} \delta_{n N} \phi_{n d}(y)$ and $\mu_{d} C_{n m}^{+} \delta_{n i} \cdot \phi_{n d}(y)$, replaced by

$$
\mu_{d} \delta n\left[C_{n+m m^{-}} \phi_{n d}(y)-U_{m}{ }_{n d}+\frac{D}{2}(y)\right]
$$

and

$$
\mu_{d} \delta_{n 1}\left[\mathrm{~V}_{m} \phi_{n d-\frac{D}{2}}(y)-C_{n m}^{+} \phi_{n d}(y)\right]
$$

respectively. The boundary conditions replacing Equations $3.46 \mathrm{~g}$ and $3.46 \mathrm{~h}$ are

$$
\begin{gathered}
\phi_{n d}\left(y_{1}\right)=\phi_{n d}+\frac{D}{4}\left(y_{1}\right) ; \eta_{d}>0 \\
\phi_{n d}\left(y_{M+1}\right)=\phi_{n d}-\frac{D}{4}\left(y_{M+1}\right) ; \eta_{d}<0
\end{gathered}
$$

The solution to the equations in this form are found in identically the same manner as described in Chapter III. 


\section{BIBLTOGRAPHY}

1. S. G. Mykhlin, Variational Methods in Mathematical Physics, p. 278, Pergamon Press, New York (1964).

2. E. I. Wachspress, "Variational Methods in Neutron Flux Synthesis," KAPL-P-3803, Knolls Atomic Power Laboratory (1969).

3. D. S. Selengut, "Nuclear Physics Research Quarterly Report," HW-59126, pp. 89-124, Hanford Atomic Products Operations (1958).

4. A. R. Schiff, "Synthesis of Three-Dimensional Power Distributions for a Non-Uniformly Depleted Core," American Nuclear Society Abstracts 3rd Meeting, Pittsburgh, Pennsylvania, Paper 1-7 (1957).

5. R. W. Devlin and R. R. Thomson, "A Method of Obtaining Axial F'lux Distributions in a Rod Programmed Core," American Nuclear Soliety Abstracts 3rd Meeting, Pittsburgh, Pennsylvania, Paper 12-9 (1957).

6. J. E. Meyer, "Synthesis of Three-Dimenisional Power Shapes," Proceedings of the Second International Conference on Peaceful Uses of Atomic Energy, Geneva, pp. 519-522 (1958).

7. E. L. Wachspress, R. D. Burgess and S. Baron, Nucl. Sci. and Eng., 12, $381(1962)$.

8. S. Kaplan, Advances in Nuclear Science and Technology, 3, P. Greebler and E. J. Henley, eds., pp. 233-266, Academic Press, New York (1966).

9. J. D. Jenkins, personal communication (1969).

10. P. Greebler, M. D. Kelley, R. A. Davis, C. A. Keck and W. A. Duncan, "BISYN -- A Two-Dimensional Synthesis Program," GEAP-4922, General Electric Company (1965).

11. "Comparison of Two Sodium-Cooled 1000 Mwe Fast Reactor Concepts, Task I Report of 1000 Mwe LMFBR Follow-On Work," GEAP-5618, pp. 215-218, General Electric Company (1968).

12. "ROD, A Reactor Optimization Design Code," ORNL Report (unpublished), Oak Ridge National Laboratory (1968).

13. S. Kaplan, 0. J. Marlowe and J. Bewick, Nucl. Sci. Eng., 18, 163 (1964).

14. J. B. Yasinsky, Nucl. Sci. Eng., 29, 381 (1967). 
15. W. M. Stacey, Jr., Nucl. Sci. Eng., 34, 45 (1968).

16. W. M. Stacey, Jr., "Multichannel Variational space-Time Synthesis with Discontinuous Expansion Functions," KAPL-M-6711, Knolls Atomic Power Laboratory (1967).

17. F. E. Smith, "Comparison of Physics Measurements and Weighted Residual Synthesis Calculations for the Shippingport PWR Core 2 Seed 1 Through Design Life," WAPD-T-2152, Bettis Atomic Power Laboratory (1968).

18. G. P. Calame and F. D. Federighi, Nucl. Sci. Eng., 10, 190 (1961).

19. F. Storrer and J. M. Chaumont, "The Application of Space-Energy Synthesis to the Interpretation of Fast Multizone Critical Experiments," Proceedings of the International Conference on Fast Critical Experiments and Their Analysis, ANL-7320, pp. 439447, Argonne National Laboratory (1966).

20. T. E. Murley and J. W. Williamson, Trans. Am. Nucl. Soc., 1l, No. 1,174 (1968).

21. A. F. Henry, Nucl. Sci. Eng., 27, 493 (1967).

22. A. J. Buslik, "Interface Conditions for Few Group Equations with Flux-Adjoint Weighted Constants," WAPD-TM-733, Bettis Atomic Power Laboratory (1968).

23. J. A. Davis and S. Kaplan, "Transport Synthesis," WAPD-T-1849, Bettis Atomic Power Iaboratory (1965).

24. S. Kaplan, J. A. Davis and M. Natleson, Nucl. Sc1. Eng., 28, 364 (1967).

25. M. Natleson, Nucl. Sci. Eng., 31, 336 (1968).

26. B. D. O'Reilly, "A Variational Synthesis Method for Calculating Structure Shielding Data," AI-68-51, Atomics International Division of North American Rockwell Corporation (1968).

27. M. Natleson and E. M. Gelbard, Trans. Am. Nucl. Soc., 12, No. 2, 728 (1969).

28. H. S. Zwibel and B. Bowes, Trans. Am. Nucl. Soc., 11, No. 1, 174 (1968).

29. V. Luco, "Space-Time Flux Synthesis Methods for the Approximate Solution of the Time Dependent Boltzmann Neutron Transport Equation," NAA-SR-11821, Atomics International (1966). 
30. M. J. Lancefield, Nucl. Sci. Eng., 37, 423 (1969).

31. B. Davidson, Neutron Transport Theory, pp. 381-389, Oxford Press, London (1957).

32. S. Kaplan, Nucl. Sci. Eng., 柿, 76 (1968).

33. P. Roussopoulos, Comptes Rendus, 236, 1858 (1953).

34. G. C. Pomraning, Nucl. Sci. Eng., 29, 220 (1967).

35. J. Lewins, Nucl. Sci. Eng., 31, 160 (1968).

36. M. Becker, The Principles and Applications of Variational Methods, The M.I.T. Press, Cambridge, Massachusetts (1964).

37. A. J. Buslik, Trans. Am. Nucl. Soc., 9, No. 1, 199 (1966).

38. E. L. Wachspress and M. Becker, "Variational Multichannel synthesis with Discontinuous Tr1al Functions," KAPL-3095, Krolls Atomic Power Laboratory (1965).

39. G. C. Pomraning, Nucl. Sc1. Eng., 28, 150 (1967).

40. S. R. Dwivedi, Nucl. Sc1. Eng., 31, $17^{4}$ (1968).

41. S. Kaplan, Advances in Nuclear Science and Technology, 5, E. J. Henley and J. Lewins, eds., pp. 185-221, Academic Presis, New York (1969).

42. J. A. Davis, Nucl. Sci. Eng., 25, 189 (1966).

43. S. Kaplan, Nucl. Sci. Eng., 34, 76 (1968).

44. F. R. Mynatt, "Development of Two-Dimensional Discrete Ordinates Transport Theory for Radiation Shielding," CTC-INF-952, Union Carbide Corporation, Nuclear Division (1969).

45. T. B. Fowler and D. R. Vondy, "Nuclear Reactor Core Analysis Code: CITATION," ORNL-TM-2496, Rev. 1, Oak Ridge National Laboratory (1970).

46. W. W. Engle, "ANISN -- A One-Dimensional Discrete Ordinates Transport Code," K-1693, Union Carbide Corporation, Nuclear Division (1967).

47. F. R. Mynatt, "A User's Manual for DOT," K-1694, Union Carbide Corporation, Nuclear Division (1968). 
48. T. B. Fowler, M. L. Tobias and D. R. Vondy, "ExTERMINAToR-2: A Fortran IV Code for Solving Multigroup Neutron Diffusion Equations in Two Dimensions," ORNL 4078 , Oak Ridge National Laboratory (1967).

49. E. L. Wachspress, "Argonne Code Center: Benchmark Problem Book," ANL-7416, p. 51, Argonne National Laboratory (1968).

50. E. M. Gelbard, Trans. Am. Nucl. Soc., 8, 226 (1965).

51. K. D. Lathrop, Nucl. Sci. Eng., 32, 357 (1968). 
VITA

William Ronald Cobb was born In Hawkinsville, Georgia, on December 22, 1944. Having moved to Atlanta at an early age, he attended public schools there and was graduated as salutatorian from Chamblee High School in 1962.

Mr. Cobb entered the Georgia Institute of Technology in 1962 as the recipient of a tuition scholarship from the Greater Atlanta Georgia Tech Alumni Association and received the Bachelor of Sclence degree in Physics with honors in 1966. During his undergraduate career Mr. Cobb was honored by membership in the Phi Kappa Phi, Tau Beta Pi, Sigma Pi Sigma, and Phi Eta Sigma honor socleties.

Mr. Cobb was awarded a National Science Foundation grant for graduate study in Nuclear Engineering and pursued a Master of Science degree in this field at Georgia Tech. Completing requirements for the degree in 1967, he was employed by the Union Carbide Corporation at the Oak Ridge National Laboratory. At ORNL, Mr. Cobb has worked in the Reactor Analysis Department, Reactor Division, in the general area of nuclear reactor physics, and in this position, has contributed to the core design of the Molten-Salt Breeder Reactor and the Molten-Salt; Breeder Experiment. He has also been associated with the reactor physics code development effort in support of the Iiquid Metal Fast Breeder Reactor Program and, in this respect, has contributed to the development of computational methods for reactor physics problems 
using advanced computers. He is presently involved in criticality safety work in support of the Aqueous Reprocessing Program for IMFBR fuels.

$\mathrm{Mr}$. Cobb is married to the former Jeanette Gagliardy of Atlanta; they have a daughter, Anne, age 6 months. 